\title{
Formation of Cold-Rolled Texture and Recrystallized Texture in Single Crystals of 3\% Silicon Iron* (Part I)
}

\author{
Cold-Rolled Texture-
}

\author{
By Tadami Taoka**, Ei-ichi Furubayashi*** and Shin Takeuchi***
}

\section{Synopsis}

Systematic studies were carried out on the formation of rolling textures in $3 \% \mathrm{Si}-\mathrm{Fe}$ single crystals with a number of preselected orientations. The rolling textures were determined by the X-ray pole figure method at successive stages of rolling, and the operating slip systems were determined by observation of slip bands on the rolling, side and front surfaces. Among all the crystals used, those in $\{100\}\langle 011\rangle,\{111\}\langle 211\rangle$ and $\{112\}\langle 110\rangle$ orientations maintained their initial orientation almost unchanged during rolling, while the others changed their orientation towards any one or two of the three with increasing reduction by rolling.

To describe the results comprehensively, the crystals were classified into three series, i.e., the first series consists of those crystals with a common $\{100\}$ plane parallel to the rolling plane, the second series with a common $\langle 011\rangle$ direction parallel to the transverse direction and the third series with a common $\langle 011\rangle$ direction parallel to the rolling direction. In the first series, the crystals rotated around the normal of the rolling plane $\{001\}$ towards the $\{001\}\langle 110\rangle$ orientation with increasing reduction. In the second series, the crystals oriented between $\{011\}\langle 100\rangle$ and $\{211\}\langle 111\rangle$ rotated around the transverse $\langle 011\rangle$ direction towards $\{111\}\langle 211\rangle$ and those between $\{211\}\langle 111\rangle$ and $\{100\}\langle 011\rangle$ rotated towards $\{100\}\langle 011\rangle$. While, all the crystals of the third series maintained their initial orientations almost unchanged during rolling.

The way the crystals change their orientations with reduction was semiquantilatively explained by geometrical considerations of the actually operating slip systems having large Schmid factors for compression and tension, taking account of change of their factors with rotation of the crystal. According to the geometrical calculation, the $\{211\}\langle 011\rangle$ orientation has been proved to be unconditionally stable for rolling, while the $\{100\}\langle 011\rangle$ and $\{111\}\langle 211\rangle$ orientations can not be stable unless some restrictions are imposed on the operation of slip systems.

\section{Introduction}

Many investigations have been made on the texture formation in rolled and in annealed crystals of body-centred cubic iron and iron alloys, especially $3 \%$ silicon iron, to determine the relationship between the initial orientation and the rolling texture, and that between the rolling texture and the annealing texture, since the original works on iron by Barrett and Levenson $^{1)}$ and on silicon ferrite by Decker and Harker. ${ }^{2)}$

Reports on the textures of silicon iron single crystals have been accumulated mainly by the following three groups. First, Dunn and $\mathrm{Koh}^{3), 4)}$ made an investigation on single crystals with two series of initial orientations. The one series was initially oriented with [011] direction parallel to the rolling direction, covering a $55^{\circ}$ range from $(100)[0 \overline{1}]$ to $(1 \overline{1} 1)[0 \overline{1}]$ via $(2 \overline{1} 1)[0 \overline{1}]$, as shown in Fig. 1. They concluded that these initial orientations were the stable orientations for rolling deformation. Another series was oriented with $[0 \overline{1} 1]$ direction parallel to the transverse direction, covering a $90^{\circ}$ range from (011)[100] to

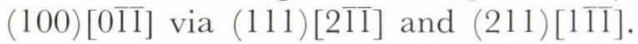

Secondly, Walter and Hibbard ${ }^{5)}$ studied on a series of crystals initially oriented with [100] direction parallel to the rolling direction and with planes such as (001), (012) and (011) in the rolling plane. They

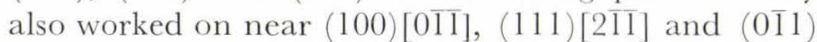
[011] crystals. The last crystal maintained its orientation near the initial orientation during rolling.

Thirdly, $\mathrm{Hu}^{6), 7)}$ reported in detail on the crystals with the same orientations as used in the series of Walter, et al. He studied especially change of rolling and annealing textures for reductions of 30,50 and $70 \%$ in thickness.

Though many experimental data have been accumulated on silicon iron single crystals with variously selected orientations, there are some discrepancies among the results reported by the different groups. For example, the relation between the rolling and the annealing textures ${ }^{5), 7), 8), 9)}$ as well as the mechanism of formation of each texture itself $\left.\left.{ }^{6}\right), 10\right), 11$ ) are described in different ways. These circumstances suggest that the final results delicately depend on specimen preparation and processing conditions, though the rolling and annealing textures were found to be primarily related to the initial orientation of the crystal.

These works have been done on crystals with diverse silicon contents prepared by different methods and under different conditions of rolling and annealing. Systematic studies of the effect of the reduction on the rolling textures and on the subsequent annealing textures are still lacking for single crystals that are prepared in exactly the same condition but into various orientations. Moreover, few observations have been made on fine structures of rolled crystals which may essentially affect the recrystallization behaviours, though some transmission electron microscopic studies of rolling and annealing textures have been done recently on crystals with some typical orientations. ${ }^{12)-17)}$

Thus, the following attentions were given throughout the present experiment.

(1) Large single crystals were prepared from one ingot of $3 \%$ silicon iron with high grade commercial purity by the strain-anneal method. From considerations on the symmetry of slip directions and the stability of orientation for rolling, the initial orientation of the crystals were preselected so as to be representative of any orientation, as shown schematically in Fig. 1.

(2) Most of the crystals were rolled at room tem-

* Received July 18, 1966.

** Tokyo Research Institute, Yawata Iron \& Steel Co., Ltd., Ida, Kawasaki.

*** National Research Institute for Metals, Nakameguro, Meguro-ku, Tokyo. 


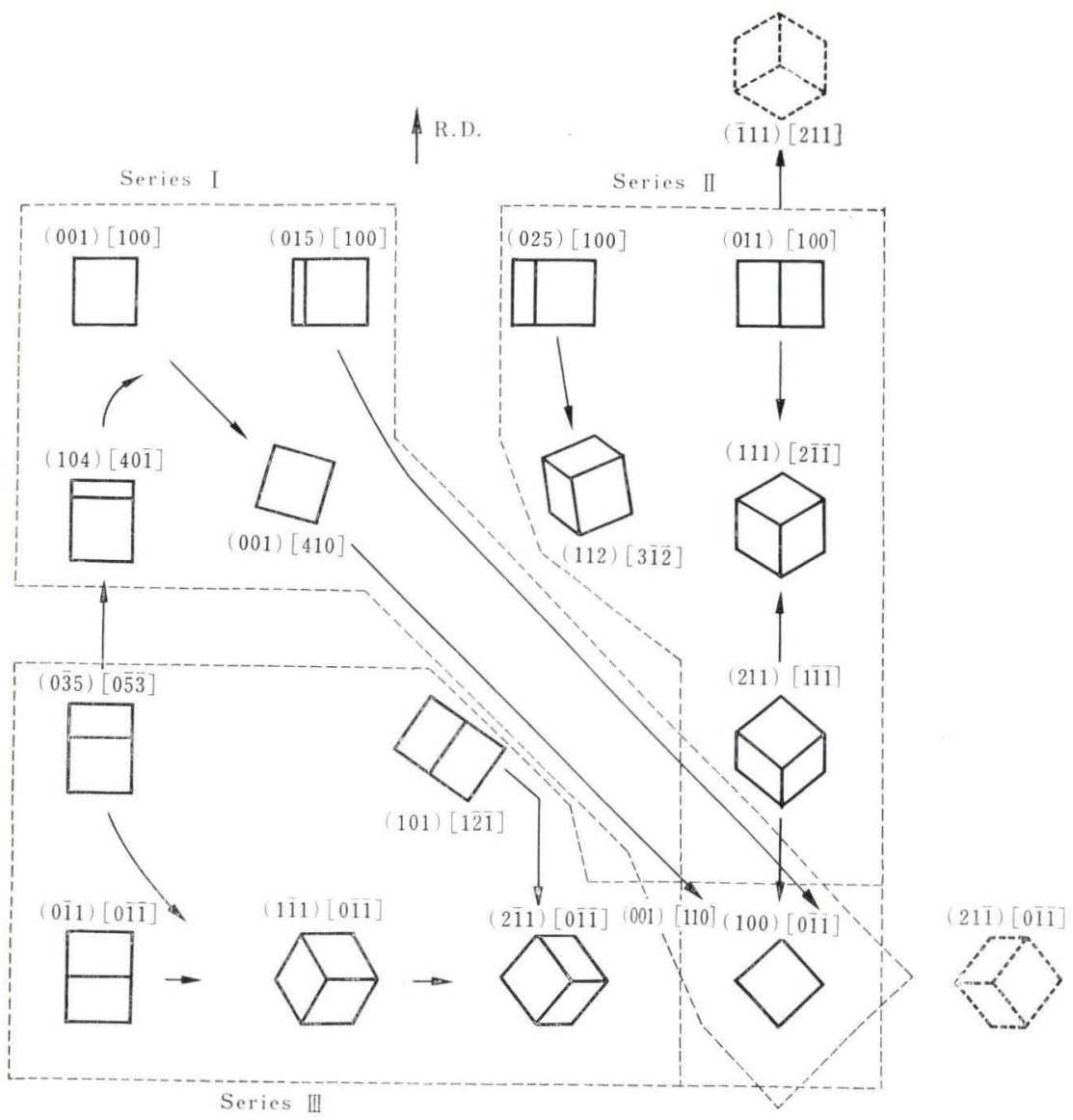

Fig. 1.

Comprehensive chart showing typical orientations of crystals used, represented schematically by unit cubes viewed along rolling plane normal, rolling direction (R.D.) being vertical. Arrows indicate orientation change due to rolling. perature to a range of reductions from 15 to $90 \%$, by about $15 \%$ reduction for each pass, in an industrial rolling mill, without reversing the direction of rolling.

(3) The rolled specimens were annealed in high purity hydrogen atmosphere for desired duration of time at various temperatures.

(4) Changes in textures were successively examined at each stage of rolling and annealing processes by the usual X-ray pole figure technique. Slip patterns and microstructures were also observed at each stage.

The first part of the present work is concerned with semi-quantitative studies on the formation of textures at successive stages of rolling process of $3 \%$ silicon iron crystals with preselected orientations, so as to have a better understanding on the mechanisms of texture formation in rolling. The studies on the formation of recrystallized textures will be reported in the second part.

\section{Experimental Procedure}

\section{Preparation of Single Crystals}

Large single crystals with preselected orientations were prepared from $3 \%$ silicon iron strips of high grade commercial purity by the usual strain-anneal method combined with a 'seed crystal' reorientation technique, as described elsewhere. ${ }^{18}$ ) Most of the single crystal strips were of 0.6 to $1 \mathrm{~mm}$ thick, as large as $30 \mathrm{~mm}$ in width and more than $150 \mathrm{~mm}$ in length, which were large enough to roll under a controlled condition in an industrial mill.

Orientations of the single crystals were selected in view of the symmetry of slip directions $\langle 111\rangle$ as well as of the end orientations for rolling, so far reported as stable ${ }^{1), 4)}$, namely $\{100\}\langle 011\rangle,\{111\}\langle 211\rangle$ and $\{111\}\langle 011\rangle$. The initial orientations of the crystals used are presented schematically in Fig. 1, where the cubes represent the typical orientations. The crystals are divided into three main series as follows :

(1) The first series, denoted by $S I$, consists of crystals with (001) plane parallel to the rolling plane. This series covers a $45^{\circ}$ range from (001)[100] to (001) [110] via (001)[410]. Crystals oriented near

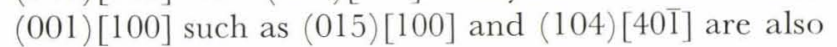
included in this series.

(2) The second series, SII, consists of crystals with [011] direction parallel to the transverse direction. This series covers a $90^{\circ}$ range from $(011)[100]$ to

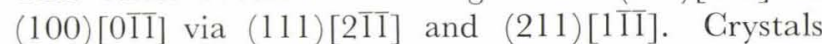
oriented near (011)[100] such as (025) [100] are also included in this series.

(3) The third series, SIII, consists of crystals with [011] direction parallel to the rolling direction. This series covers a $90^{\circ}$ range from $(100)[0 \overline{1}]$ to $(0 \overline{1} 1)$ [011]. Crystals oriented near $(0 \overline{1} 1)[0 \overline{11}]$ such as (035) $[0 \overline{5} \overline{3}]$ and $(101)[1 \overline{2} \overline{1}]$ are included in this series.

Table 1. Chemical compositions (wt $\%$ )

\begin{tabular}{c|c|c|c|c|c}
\hline Specimen & Si & C & Mn & P & S \\
\hline Polycrystalline strip & 2.80 & 0.017 & 0.04 & 0.015 & 0.014 \\
\hline Single crystal & 2.68 & 0.001 & 0.035 & 0.005 & 0.002
\end{tabular}


Table 2. Initial orientations of crystals and their rolling procedures

\begin{tabular}{|c|c|c|c|c|c|c|}
\hline \multirow{2}{*}{$\begin{array}{l}\text { Series and } \\
\text { specimen } \\
\text { number }\end{array}$} & \multirow{2}{*}{$\begin{array}{c}\text { Initial orientation } \\
\theta^{*}, \varphi^{* *}(\mathrm{deg})\end{array}$} & \multicolumn{2}{|c|}{ Rolling condition } & \multicolumn{2}{|c|}{ Strip size } & \multirow[b]{2}{*}{ Symbol } \\
\hline & & $\begin{array}{l}\text { Reduction } \\
(\%)\end{array}$ & $\begin{array}{l}\text { Number } \\
\text { of pass }\end{array}$ & $\underset{(\mathrm{mm})}{\text { Thickness }}$ & $\begin{array}{l}\text { Width (mm) or its } \\
\text { widening (\%) }\end{array}$ & \\
\hline \multirow{10}{*}{$S I-77$} & \multirow{10}{*}{$\frac{(001)}{2^{\circ}}$} & & & 0.86 & $27.3 \mathrm{~mm}$ & \\
\hline & & 24 & 1 & 0.65 & $0.0 \%$ & \\
\hline & & 51 & 2 & 0.42 & $+2.9 \%$ & \\
\hline & & 75 & 6 & 0.205 & $+4.0 \%$ & \\
\hline & & 86 & 10 & 0.115 & $+5.2 \%$ & \\
\hline & & 95 & 25 & 0.043 & $+6.6 \%$ & \\
\hline & & 50 & 8 & 0.42 & $+1.8 \%$ & \\
\hline & & 65 & 15 & 0.29 & $+1.8 \%$ & \\
\hline & & 76 & 21 & 0.19 & $+2.9 \%$ & \\
\hline & & 85 & 29 & 0.12 & $+7.0 \%$ & \\
\hline \multirow{4}{*}{$S I-33$} & \multirow{4}{*}{$\begin{array}{c}(001) \\
2^{\circ}\end{array}$} & & & 0.78 & $18.0 \mathrm{~mm}$ & \\
\hline & & 29 & 2 & 0.556 & $+2.2 \%$ & \\
\hline & & 60 & 4 & 0.30 & $+1.1 \%$ & \\
\hline & & 73 & 6 & 0.19 & $-0.5 \%$ & \\
\hline \multirow{3}{*}{$S I-37$} & \multirow{3}{*}{$\begin{array}{l}(001) \\
1^{\circ}\end{array}$} & & & 0.73 & $29.1 \mathrm{~mm}$ & \\
\hline & & 29 & 2 & 0.525 & $+0.0 \%$ & \\
\hline & & 74 & 8 & 0.19 & $+2.1 \%$ & \\
\hline \multirow{4}{*}{$S I-109$} & \multirow{4}{*}{$\begin{array}{c}(001) \\
1^{\circ}\end{array}$} & & & 0.69 & $17.0 \mathrm{~mm}$ & \\
\hline & & 25 & 1 & 0.51 & $+0.0 \%$ & \\
\hline & & 56 & 2 & 0.32 & $+1.2 \%$ & \\
\hline & & 75 & 4 & 0.182 & $+5.3 \%$ & \\
\hline \multirow{2}{*}{$S I-232$} & \multirow{2}{*}{ (001) } & & & 0.8 & $30 \mathrm{~mm}$ & \\
\hline & & 74 & 8 & 0.21 & - & \\
\hline \multirow{4}{*}{$S I-107$} & \multirow{4}{*}{$\begin{array}{l}(015) \\
3.5^{\circ}\end{array}$} & & & 0.765 & $27.2 \mathrm{~mm}$ & \\
\hline & & 47 & 2 & 0.403 & $+1.4 \%$ & \\
\hline & & 74 & 7 & 0.203 & $+4.4 \%$ & \\
\hline & & 77 & 9 & 0.116 & $+5.9 \%$ & \\
\hline & & & & 0.90 & $20.6 \mathrm{~mm}$ & \\
\hline$S I-234$ & (104) [401] & 24 & 1 & 0.68 & $+2.9 \%$ & \\
\hline & & 55 & 4 & 0.40 & $+5.3 \%$ & \\
\hline & & 76 & 8 & 0.22 & $+6.8 \%$ & \\
\hline & & & & 0.75 & $27.5 \mathrm{~mm}$ & \\
\hline$S I I-108$ & $(011) \quad[100]$ & 25 & 1 & 0.565 & $-0.4 \%$ & \\
\hline & $5.5^{\circ}$ & 58 & 5 & 0.32 & $+0.6 \%$ & \\
\hline & & 75 & 10 & 0.18 & $+3.6 \%$ & \\
\hline & & & & 0.603 & $26.7 \mathrm{~mm}$ & \\
\hline$S I I-23$ & $(011) \quad[100]$ & 47 & 2 & 0.32 & $+0.7 \%$ & \\
\hline & & 71 & 10 & 0.175 & $+6.1 \%$ & \\
\hline & & 86 & 25 & 0.087 & $+11.6 \%$ & \\
\hline$S I I-31$ & $(011) \quad[100]$ & & & 0.62 & $31.0 \mathrm{~mm}$ & \\
\hline & & 8.2 & 1 & 0.57 & $+0.0 \%$ & \\
\hline$S I I-29$ & (011) [100] & & & 0.48 & $18.3 \mathrm{~mm}$ & \\
\hline & $3.5^{\circ}$ & 21 & 2 & 0.38 & $+3.1 \%$ & \\
\hline
\end{tabular}


Continued from last page

\begin{tabular}{|c|c|c|c|c|c|c|c|}
\hline \multirow{2}{*}{$\begin{array}{l}\text { Series and } \\
\text { specimen } \\
\text { number }\end{array}$} & \multirow{2}{*}{\multicolumn{2}{|c|}{$\begin{array}{c}\text { Initial orientation } \\
\theta^{*}, \varphi^{* *}(\mathrm{deg})\end{array}$}} & \multicolumn{2}{|c|}{ Rolling condition } & \multicolumn{2}{|c|}{ Strip size } & \multirow{2}{*}{ Symbol } \\
\hline & & & $\begin{array}{c}\text { Reduction } \\
(\%)\end{array}$ & $\begin{array}{c}\text { Number } \\
\text { of pass }\end{array}$ & $\begin{array}{c}\text { Thickness } \\
(\mathrm{mm})\end{array}$ & $\begin{array}{l}\text { Width }(\mathrm{mm}) \text { or its } \\
\text { widening }(\%)\end{array}$ & \\
\hline \multirow{3}{*}{$S I I-33$} & \multirow{3}{*}{$\left(3^{\circ}\right)$} & \multirow{3}{*}{$\frac{[100]}{3^{\circ}}$} & & & 0.62 & $30 \mathrm{~mm}$ & \\
\hline & & & 32 & 1 & 0.42 & - & \\
\hline & & & 58 & 3 & 0.26 & - & \\
\hline \multirow{2}{*}{$S I I-37$} & \multirow{2}{*}{$\stackrel{(011)}{2^{\circ}}$} & \multirow{2}{*}{$\begin{array}{l}{[100]} \\
3^{\circ}\end{array}$} & & & 0.62 & $27.0 \mathrm{~mm}$ & \\
\hline & & & 71 & 7 & 0.183 & $+2.6 \%$ & \\
\hline \multirow{5}{*}{$S I I-104$} & \multirow{5}{*}{$\begin{array}{c}(233) \\
6^{\circ}\end{array}$} & \multirow{5}{*}{$\begin{array}{l}{[3 \overline{11}]} \\
2.5^{\circ}\end{array}$} & & & 0.87 & $28.1 \mathrm{~mm}$ & \\
\hline & & & 31 & 2 & 0.60 & $-0.4 \%$ & \\
\hline & & & 52 & 4 & 0.42 & $+0.7 \%$ & \\
\hline & & & 73 & 9 & 0.23 & $+4.3 \%$ & \\
\hline & & & 79 & 13 & 0.194 & $+6.1 \%$ & \\
\hline \multirow{3}{*}{$S I I-214$} & \multirow{3}{*}{$\begin{array}{l}(233) \\
0.5^{\circ}\end{array}$} & \multirow{3}{*}{$\begin{array}{l}{[3 \overline{1} \overline{1}]} \\
5^{\circ}\end{array}$} & & & 0.85 & $29.2 \mathrm{~mm}$ & \\
\hline & & & 42 & 3 & 0.50 & $+1.7 \%$ & \\
\hline & & & 60 & 6 & 0.34 & $+4.1 \%$ & \\
\hline \multirow{2}{*}{ S II-102 } & \multirow{2}{*}{$\begin{array}{l}(111) \\
2.5^{\circ}\end{array}$} & \multirow{2}{*}{$\begin{array}{l}{[2 \overline{1} \overline{1}]} \\
6^{\circ}\end{array}$} & & & 0.85 & $28.3 \mathrm{~mm}$ & \\
\hline & & & 74 & 8 & 0.23 & $+3.2 \%$ & \\
\hline \multirow{3}{*}{ S II-302 } & \multirow{3}{*}{$\begin{array}{l}(111) \\
2.5^{\circ}\end{array}$} & \multirow{3}{*}{$\begin{array}{l}{[2 \overline{1} \overline{1}]} \\
5^{\circ}\end{array}$} & & & 0.83 & $28.3 \mathrm{~mm}$ & \\
\hline & & & 9 & 1 & 0.76 & $+0.0 \%$ & \\
\hline & & & 72 & 9 & 0.23 & $+5.9 \%$ & \\
\hline & & & & & 0.81 & $28.5 \mathrm{~mm}$ & \\
\hline$S I I-304$ & $\frac{(322)}{1^{\circ}}$ & $\begin{array}{c}{[4 \overline{3} \overline{3}]} \\
0^{\circ}\end{array}$ & 9 & 1 & 0.74 & $+0.3 \%$ & \\
\hline & & & 74 & 8 & 0.24 & $+7.0 \%$ & \\
\hline & & & & & 0.83 & $28.1 \mathrm{~mm}$ & \\
\hline & & & 17 & 1 & 0.685 & $+0.7 \%$ & \\
\hline$S I I-303$ & $\frac{(211)}{4^{\circ}}$ & $\begin{array}{l}{[1 \overline{11}]} \\
0.5^{\circ}\end{array}$ & 37 & 2 & 0.52 & $+1.4 \%$ & \\
\hline & & & 57 & 4 & 0.36 & $+2.6 \%$ & \\
\hline & & & 75 & 7 & 0.21 & $+4.6 \%$ & \\
\hline & & & & & 0.75 & $20.0 \mathrm{~mm}$ & \\
\hline$S I I-112$ & $\begin{array}{c}(511) \\
1^{\circ}\end{array}$ & $\begin{array}{l}{[25 \overline{5}]} \\
1.5^{\circ}\end{array}$ & 22 & 1 & 0.58 & $+0.9 \%$ & \\
\hline & & & 72 & 7 & 0.21 & $+8.4 \%$ & \\
\hline & & & & & 0.79 & $28.7 \mathrm{~mm}$ & \\
\hline & & & 10 & 1 & 0.69 & $+0.0 \%$ & \\
\hline$S I I-111$ & $\begin{array}{c}(025) \\
3^{\circ}\end{array}$ & ${ }_{7.5^{\circ}}^{[100]}$ & 48 & 3 & 0.40 & $+0.7 \%$ & \\
\hline & & & 73 & 8 & 0.21 & $+4.5 \%$ & \\
\hline & & & 77 & 10 & 0.175 & $+5.9 \%$ & \\
\hline$S I I I-215$ & $(2 \overline{1} 1)$ & [01̄ī] & & & 0.91 & $29.1 \mathrm{~mm}$ & \\
\hline & & & 78 & 9 & 0.20 & $+3.7 \%$ & \\
\hline & & & & & 0.86 & $29.0 \mathrm{~mm}$ & \\
\hline$S I I I-210$ & $\left(\begin{array}{l}(111) \\
4^{\circ}\end{array}\right.$ & {$\left[\begin{array}{c}{[0 \overline{1} \overline{1}]} \\
3^{\circ}\end{array}\right.$} & 55 & 4 & 0.41 & - & \\
\hline & & & 74 & 8 & 0.22 & $+6.2 \%$ & \\
\hline & & & & & 0.69 & $29.2 \mathrm{~mm}$ & \\
\hline$S I I I-108$ & $(0 \overline{1} 1)$ & {$[0 \overline{1} \overline{1}]$} & 6 & 1 & 0.648 & $+2.8 \%$ & \\
\hline & 3.5 & & & & 0.77 & $35.3 \mathrm{~mm}$ & \\
\hline & & & 71 & 5 & 0.226 & $-7.7 \%$ & \\
\hline
\end{tabular}


Continued from last page

\begin{tabular}{|c|c|c|c|c|c|c|}
\hline \multirow{2}{*}{$\begin{array}{l}\text { Series and } \\
\text { specimen } \\
\text { number }\end{array}$} & \multirow{2}{*}{$\begin{array}{c}\text { Initial orientation } \\
\theta^{*}, \varphi^{* *}(\mathrm{deg})\end{array}$} & \multicolumn{2}{|c|}{ Rolling condition } & \multicolumn{2}{|c|}{ Strip size } & \multirow{2}{*}{ Symbol } \\
\hline & & $\underset{(\%)}{\text { Reduction }}$ & $\begin{array}{l}\text { Number } \\
\text { of pass }\end{array}$ & $\begin{array}{c}\text { Thickness } \\
(\mathrm{mm})\end{array}$ & $\begin{array}{l}\text { Width }(\mathrm{mm}) \text { or its } \\
\text { widening }(\%)\end{array}$ & \\
\hline \multirow{4}{*}{$S I I I-30$} & \multirow{4}{*}{$\begin{array}{l}(0 \overline{3} 5) \\
\left.2^{\circ}\right)\end{array}$} & & & 0.78 & $35.0 \mathrm{~mm}$ & \\
\hline & & 7 & 1 & 0.714 & $-3.5 \%$ & \\
\hline & & & & 0.78 & $35.5 \mathrm{~mm}$ & \\
\hline & & 67 & 9 & 0.252 & $+3.9 \%$ & \\
\hline \multirow{4}{*}{$S I I I-17$} & \multirow{4}{*}{$\begin{array}{l}(101) \\
12^{\circ}\end{array} \quad\left[\begin{array}{c}1 \overline{1} \overline{1} \\
5^{\circ}\end{array}\right.$} & & & 0.895 & $29.3 \mathrm{~mm}$ & \\
\hline & & 28 & 2 & 0.64 & $+0.0 \%$ & \\
\hline & & 49 & 4 & 0.46 & $+0.3 \%$ & \\
\hline & & 72 & 8 & 0.245 & $+5.1 \%$ & \\
\hline
\end{tabular}

Note: $\theta^{*}$ and $\varphi^{* *}$ are the deviation angles of rolling plane and direction from the indicated orientation, respectively.

The strips grown into single crystals were annealed at $1150^{\circ} \mathrm{C}$ and slowly cooled in dry hydrogen. Chemical analysis of specimens is given in Table 1 (see p. 291). Table 2 (see p. 292) tabulates the initial orientation, the size and the rolling procedures for all the crystals used.

\section{Rolling Procedure}

The large single crystal strips, 0.6 to $1.0 \mathrm{~mm}$ thick, were rolled at room temperature in the longitudinal direction, i.e., the growth direction, in an industrial rolling mill with working rolls of $150 \mathrm{~mm}$ in diameter, lubricated with rape oil. Each specimen was rolled to reductions ranged from 15 to $90 \%$ in thickness by about $15 \%$ for each pass without reversing the rolling direction. In preparing specimens to different reductions in thickness from the same crystal, a length was cut off the rolled specimen when a desired reduction was reached, the remainder being rolled further.

A capacitance type strain gauge which was combined with an ionization transducer was mounted under the back-up roll to measure the stress acting on the strip surface during rolling. By this device, forces up to 20 tons were converted into electric voltage by a monotonical relation. The change in hardness associated with reduction by rolling was also measured under 25-g load by a Vickers microhardness tester.

To make slip patterns visible, some single crystal strips were cut by a small handsaw to make the side surface and front surface precisely perpendicular to the rolling plane and were electropolished in Morris etchant ${ }^{19)}$ before rolling. Immediately after rolling, these specimens were dipped into benzine in order to avoid the oil etching.

\section{Observations of Structure and Texture}

The slip bands on electropolished side, front and rolling surfaces of specimens were observed by an optical microscope to determine the slip systems operated during rolling. The microstructure was examined by the usual metallographic technique. To observe the deformation structure corresponding to the local orientation changes of deformed matrix, each specimen was macroetched in an aqueous solution of ferric chloride; the attack of this reagent was very sensitive to the orientation change. For more precise determination of the local change of orientation, microetch pits were developed by proper etchants. ${ }^{20), 21)}$ The pits were composed of low index facets of an area as small as $6 \times 6 \mu^{2}$.

Textures of the cold rolled specimens were determined by the pole figure method at each stage of rolling in a G.E. X-ray Geiger counter diffractometer with an integrating sample holder, by the back-reflection method using (220) reflections of $\mathrm{FeK} \alpha$ radiation. By this method, three to five of the six (110) poles were registered within the central circle of $65^{\circ}$ radius on the stereographic polar net; the information is sufficient to analyse the texture. In some cases, the poles outside the central circle were determined by the transmission method using (110) reflections of MoK $\alpha$ radiation. Intensity contours were drawn on the pole figures as multiples of the random intensity measured on a carbonyl iron powder specimen after appropriate corrections for absorption and background intensity were made. The contour lines were taken in a logarithmic scale of pole density values, namely, 1, 2.7, $5.4,10,17,30$ and so on. The pole density values of the highest contour line are indicated in all the pole figures.

The textures of surface layer and interior were distinguished when the surface layer was etched away, because the effective depth exposed by $\mathrm{FeK} \alpha$ radiation was $20 \mu$ or less.

\section{Experimental Results}

\section{First Series, SI}

At first, a crystal with the (001) [100] orientation, SI-77, was rolled to reductions in thickness of 24, 51, 75,86 and $95 \%$. The average rolling pressure during each pass, i.e., the total rolling load divided by the contact area of specimen surface, was obtained, as well as Vickers microhardness after several of rolling steps. As shown in Fig. 2 (a), the average pressure slowly increases up to $50 \%$ reduction and then keeps constant, about $150 \mathrm{~kg} / \mathrm{mm}^{2}$, through subsequent passes. The microhardness change with reduction is in the same way as the rolling pressure.

In early stage of deformation, the slip bands could 

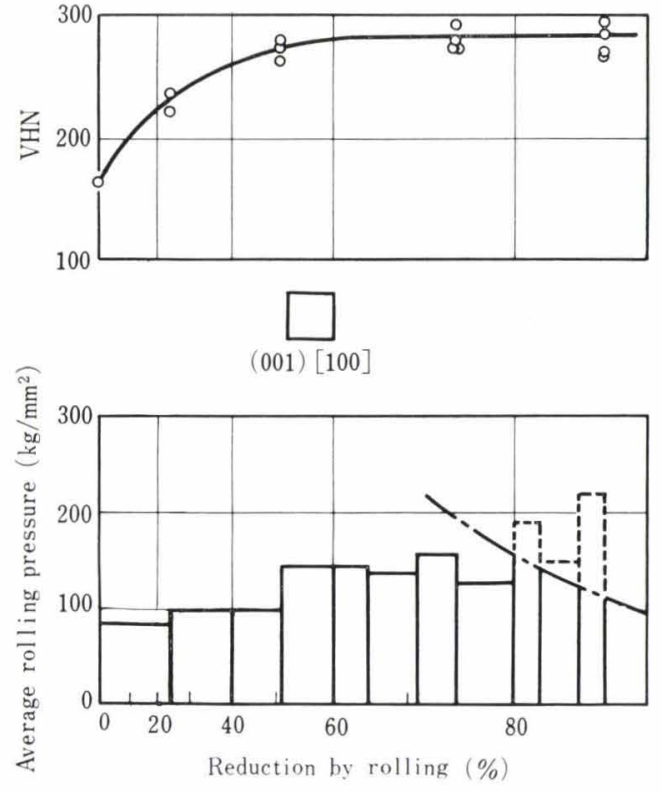

(a)

$20 \mu$

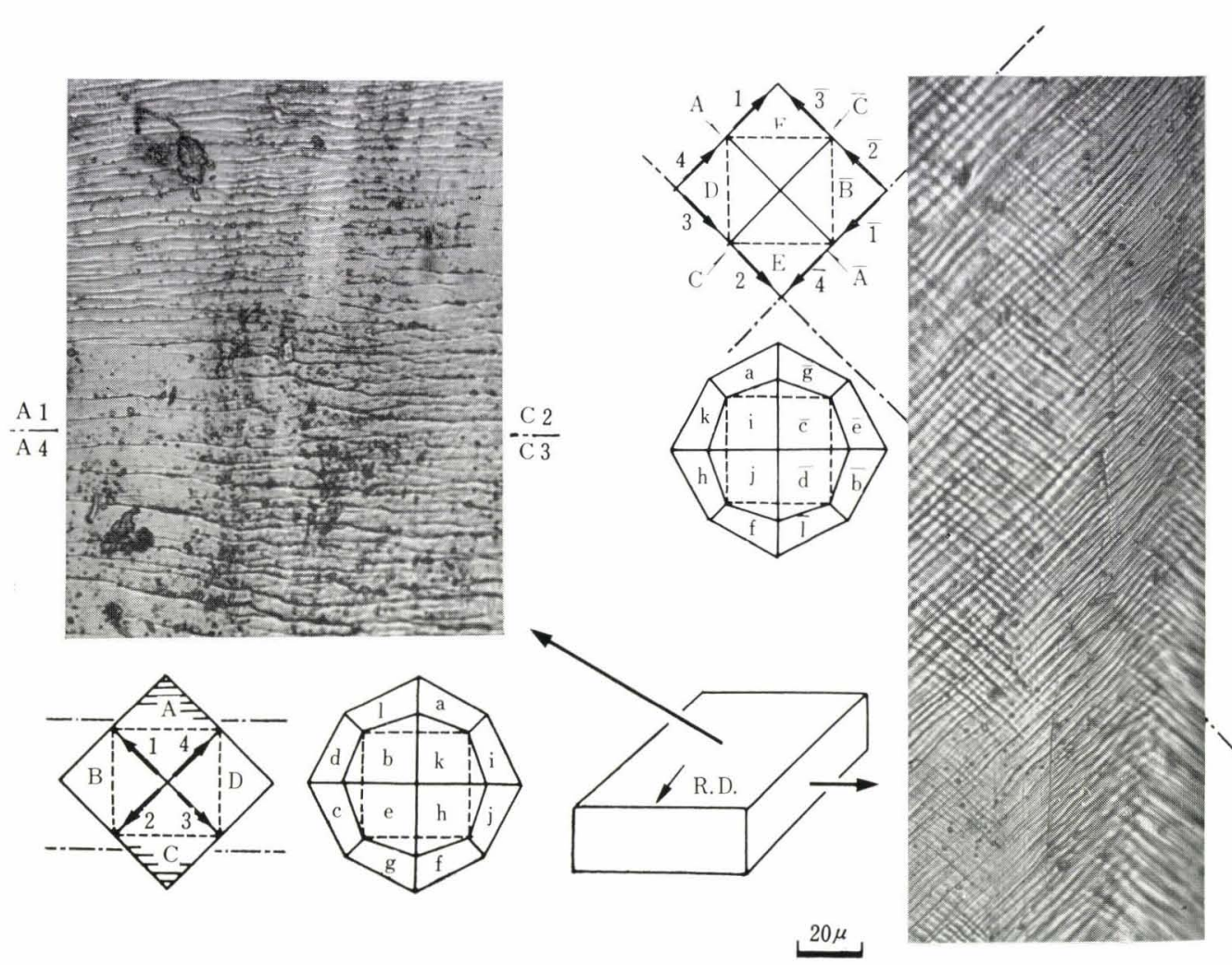

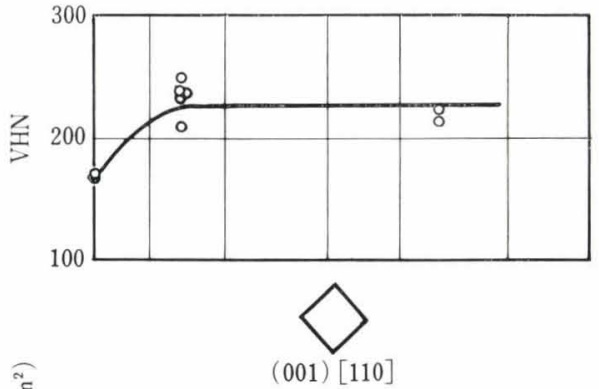

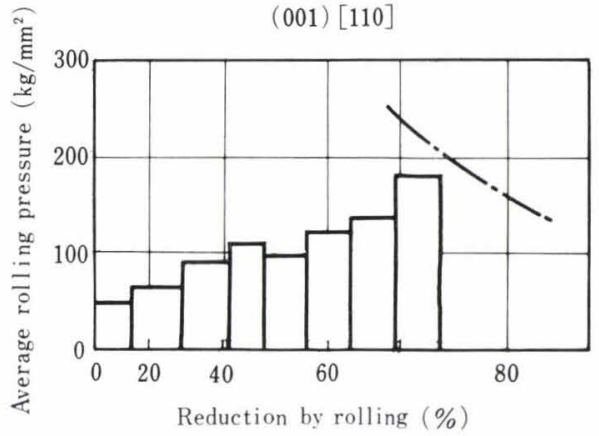

(b)

Fig. 2.

Change of average rolling pressure and Vickers micro-hardness with reduction by rolling

(a) (001) [100] crystal, $S I-77$ (b) (001) [110] crystal, SI-37

Fig. 3.

Slip patterns on rolling and side surfaces of (001)[100] crystal, $S I-77$ rolled to $24 \%$ reduction. Schematic diagrams represent crystallographic orientation, where dotted lines are projection of a cube, solid lines are that of dodecahedron composed of $\{110\}$ planes or of icositetrahedron of $\{112\}$ planes, and arrows are $\langle 111\rangle$ directions. Dot-anddashed lines show traces of operated slip planes indicated by hatching.

be clearly observed on both the rolling and side surfaces, as shown in the photographs of the (001)[100] crystal rolled to $24 \%$ reduction in Fig. 3. Referring to their respective schematic diagrams of the crystallographic orientation of those surfaces, it is found that the slip bands are parailel to traces of the $\{110\}$ planes, $A$ and $C$ identified by hatching. The slip bands could be observed even at $50 \%$ reduction; however, they were too complex to be analysed for the operating slip planes at still higher reductions. On the diagrams, figures, 1, 2, 3, 4 represent $\langle 111\rangle$ directions, capital letters, $A, B, C \ldots F,\{110\}$ planes, and small letters, $a, b, c, \ldots l,\{112\}$ planes. These notations are defined in Appendix I.

The deformation textures of the (001)[100] crystals rolled to 24,75 and $95 \%$ reduction are shown in the (110) pole figures of Fig. 4. The rolling texture spreads around the initial orientation at early stage of deformation, and then splits into two symmetrical components, the components ultimately approaching the same end orientation of $\{100\}\langle 011\rangle$. The symmetrical components are related to the initial orientation (001)[100] by rotation around the normal to the rolling plane as already reported.5),7),22) These circumstances are well presented in Fig. 5. It shows profiles of pole densities along the circle of $45^{\circ}$ radius 
on the stereographic polar net. The rotation angles of pole density maxima around the normal of the rolling plane are plotted as a function of reduction in Fig. 6. The amount of rotation remains small up to $50 \%$ reduction but increases monotonously to about

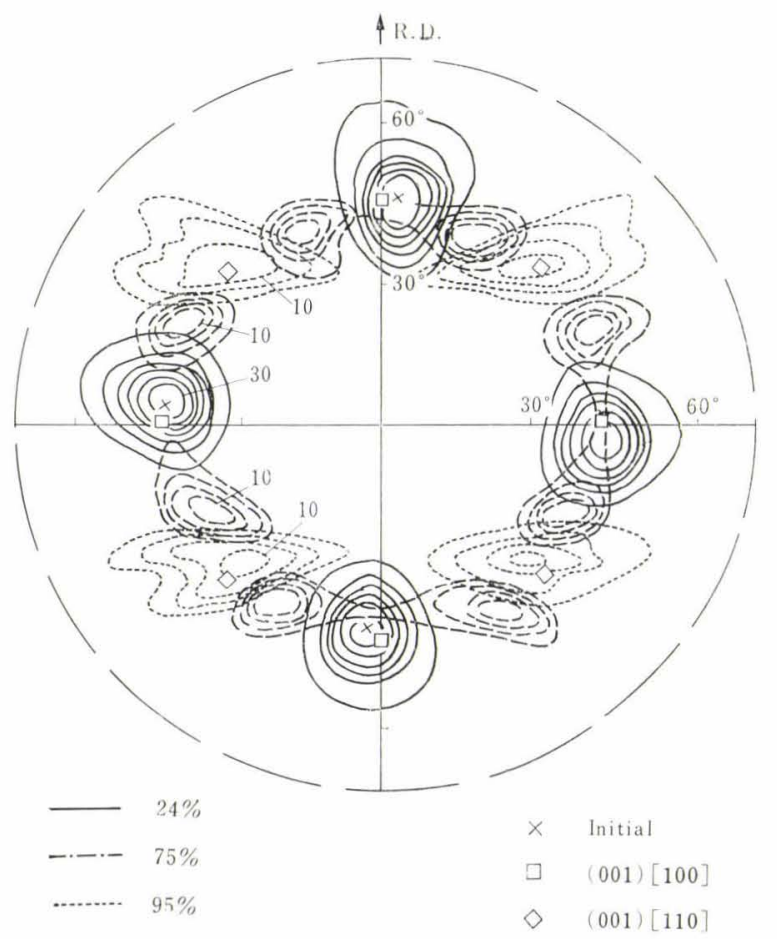

Fig. 4. (110) pole figure of (001)[100] crystal rolled to 24, 75 and $95 \%$ reduction, $S I-77$, crosses, squares and inclined squares indicate $\{110\}$ poles of initial orientation, (001)[100], and (001)[110], respectively $45^{\circ}$ from about $10^{\circ}$ at $50 \%$ reduction.

The structure revealed by etching is composed of bands elongated parallel to the rolling direction and separated by dark boundaries, as seen in Photo 1. The boundary planes were nearly parallel to the side surface. The bands were usually 20 to $200 \mu$ in width and longer than a few $\mathrm{mm}$ in length. The square shaped etch-pits appearing in each band were found to be composed of crystallographic planes. ${ }^{21)}$ The orientation of pits indicates that the alternate band is

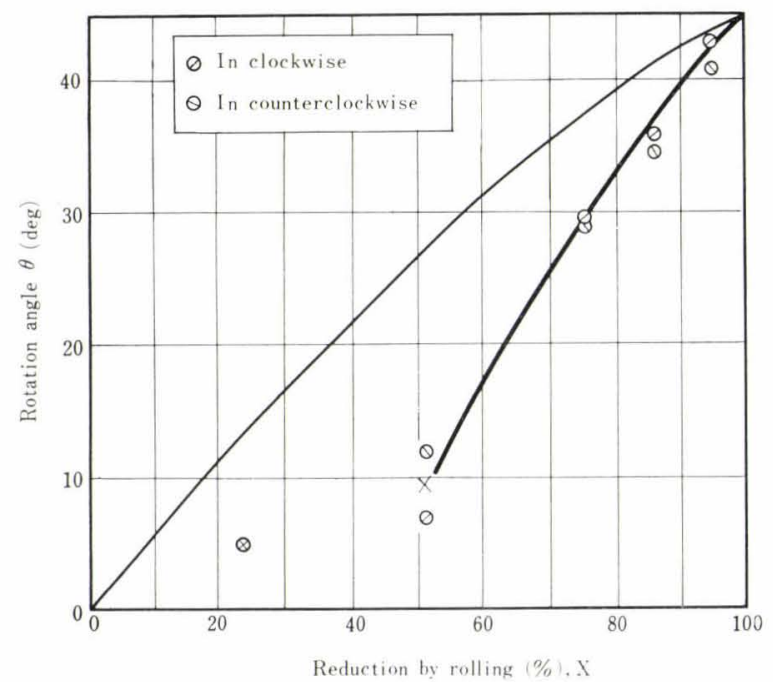

Fig, 6. Rotation angle of maximum in (110) pole density of two symmetrical components of rolling texture around normal to (001) rolling plane as a function of reduction in (001)[100] crystal, $S I-77$, together with calculated curves by a simple geometrical relation

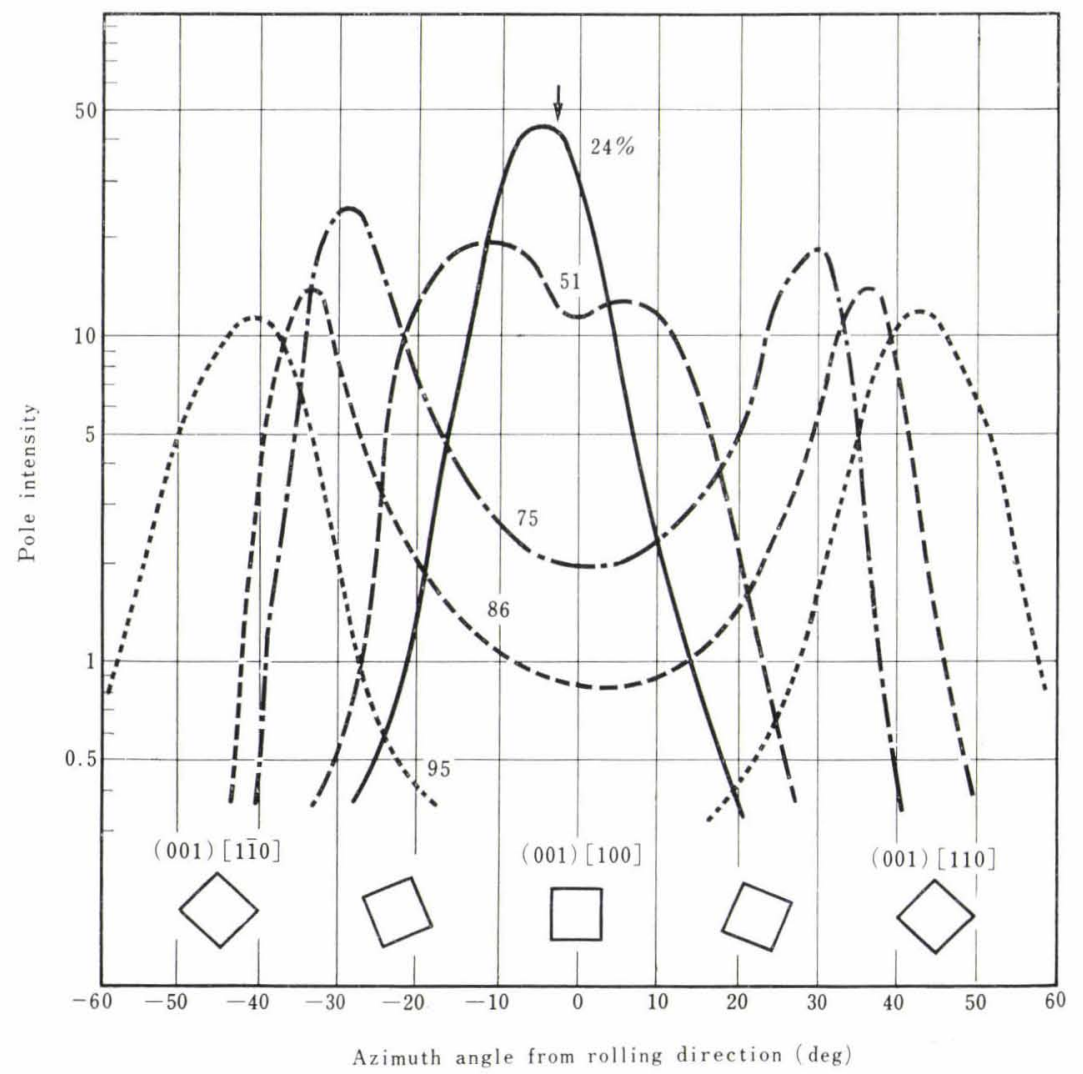

Fig. 5.

profiles of pole density along $45^{\circ}$ radius circle on the pole figure of Fig. 4, in (001)[100] crystal, $S I-77$ rolled to $24,51,75,86$ and $95 \%$ reduction 


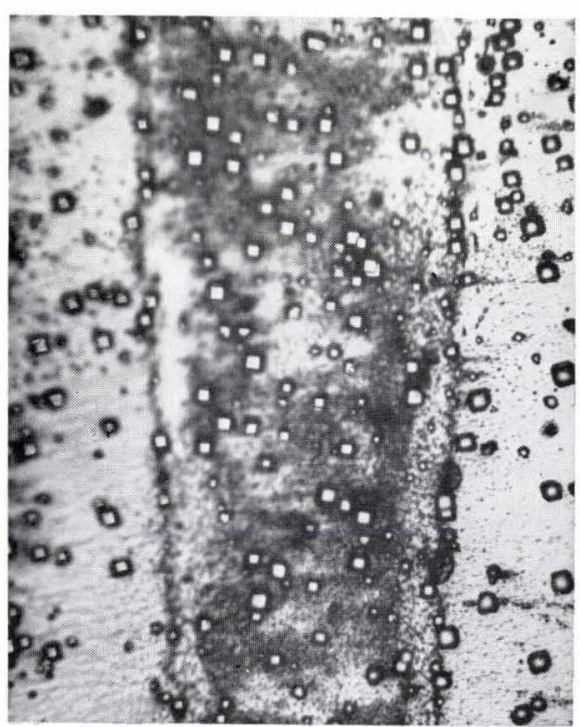

(a)

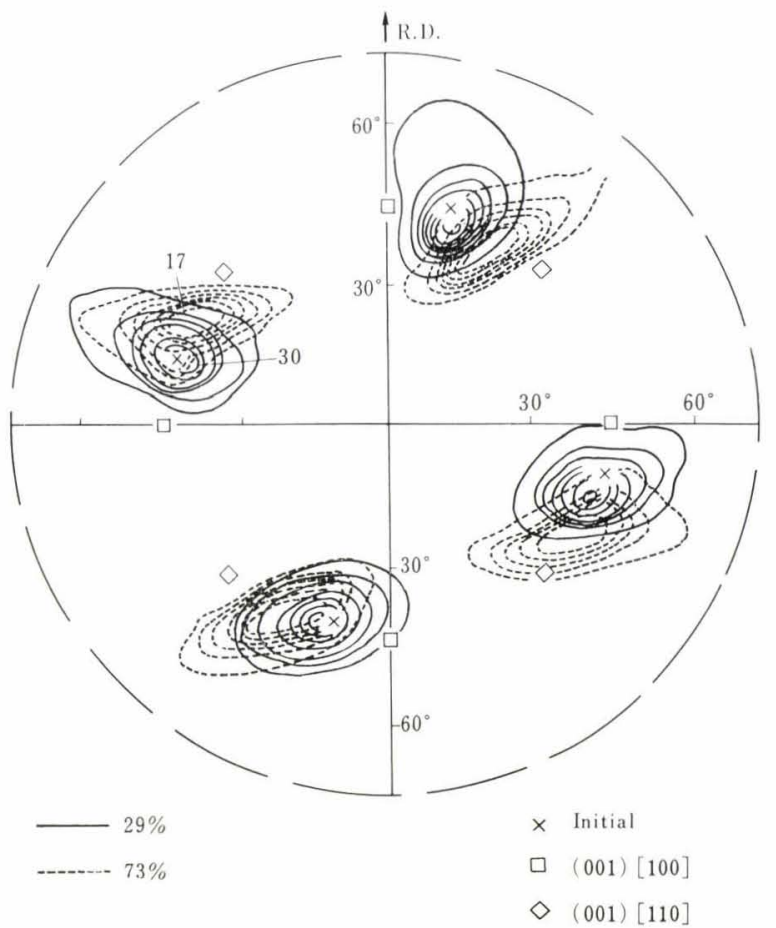

Fig. 7. (110) pole figure of (001)[410] crystal, SI-33 rolled to 29 and $73 \%$ reduction

in the same orientation and the neighbouring bands are oriented symmetrically about the boundary. The result is in agreement with the pole figure analysis that the rolling texture is composed of two symmetrical components.

Fig. 7 is the (110) pole figure of the (001)[410] crystal, SI-33, oriented between the (001)[100] and the (001) [110] stable end orientation, rolled to 29 and $73 \%$ reduction, respectively. The crystal rotates clockwise from the initial orientation around the normal to the rolling plane and approaches the end orientation with increasing reduction. The etch pit pattern on the surface shows that the rolling structure has only one component and is free from any banded structure, as seen in Photo 2. It is interesting to note
Photo 1 .

Etched patterns of (001)[100] crystal, rolled to 51 and $75 \%$ reduction, showing crystallographic orientation of each band by micro-etch pits

(a) Rolled to $51 \%$

(b) Rolled to $75 \%$

(b)

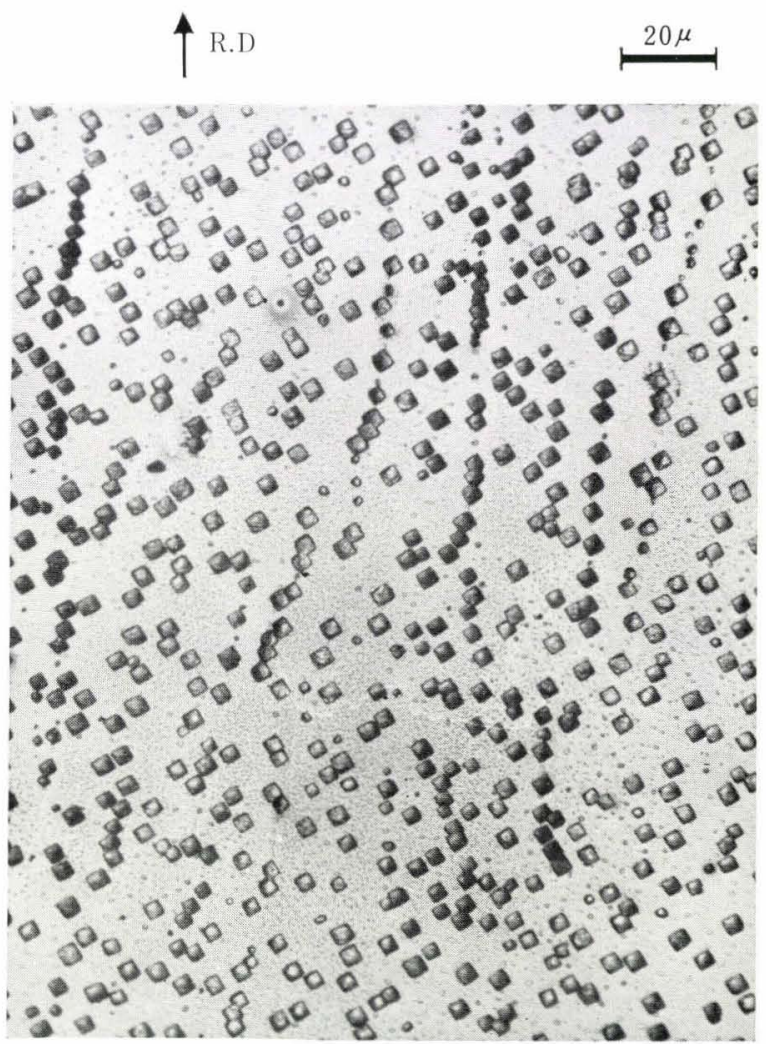

Photo 2. Micro-etch pit pattern of (001)[410] crystal, SI33 , rolled to $73 \%$ reduction

that the width of this crystal strip decreased with increasing reduction in thickness, see Table 2 . This result will be analysed later in relation to the mechanism of rolling deformation.

Next, a crystal initially oriented in (001) [110], SI37 , was rolled up to $74 \%$ reduction in thickness in 8 passes. The change of average rolling pressure during each pass is given in Fig. 2 (b), together with Vickers microhardness. The average pressure monotonously increases up to $74 \%$ reduction, while the microhardness rapidly increases at early stage of rolling and then keeps approximately constant in later 


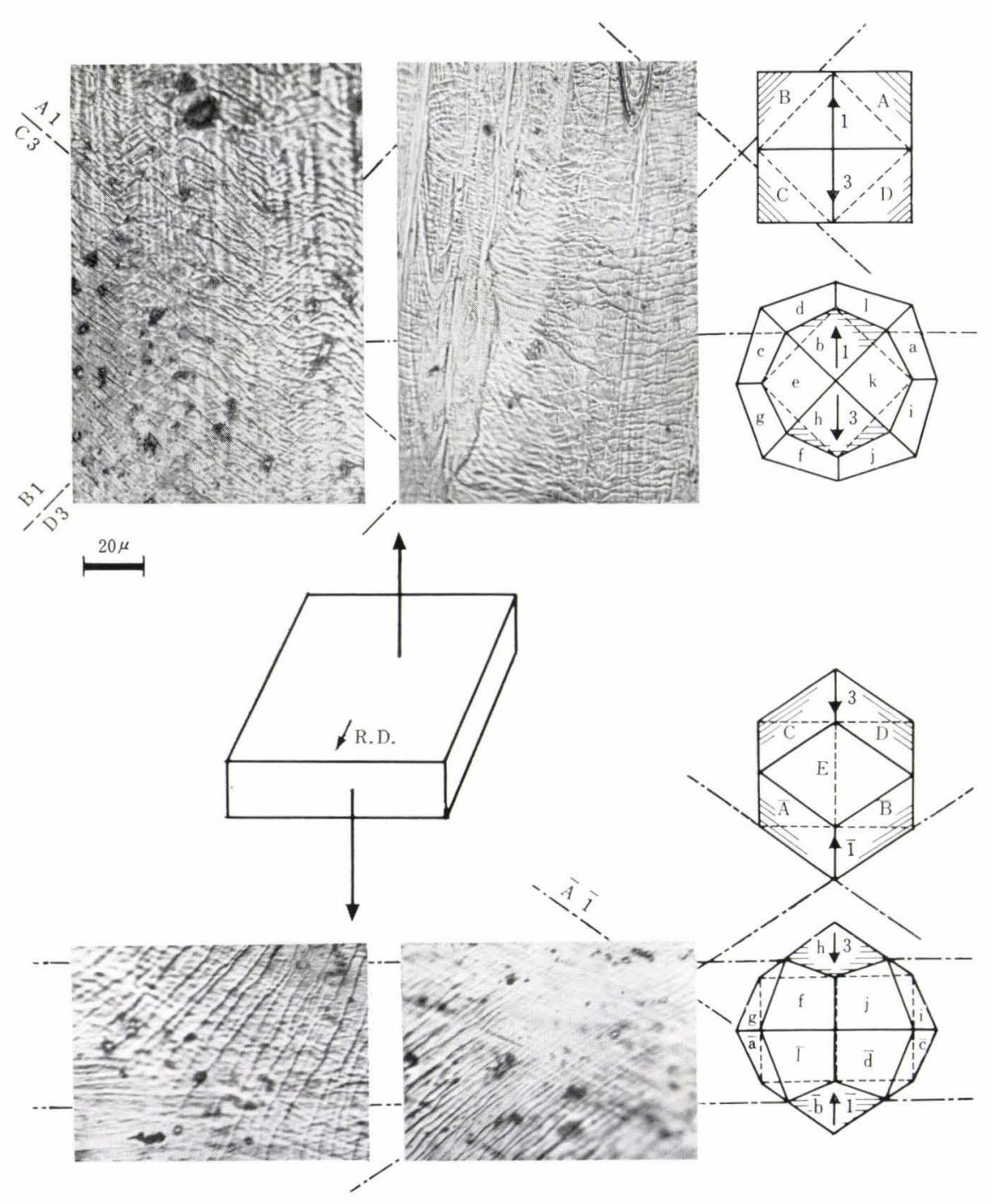

Fig. 8.

Slip patterns on front and rolling surfaces of (001)[110] crystal, $S I-37$ rolled to $29 \%$ reduction, together with schematic diagrams representing crystallographic orientations

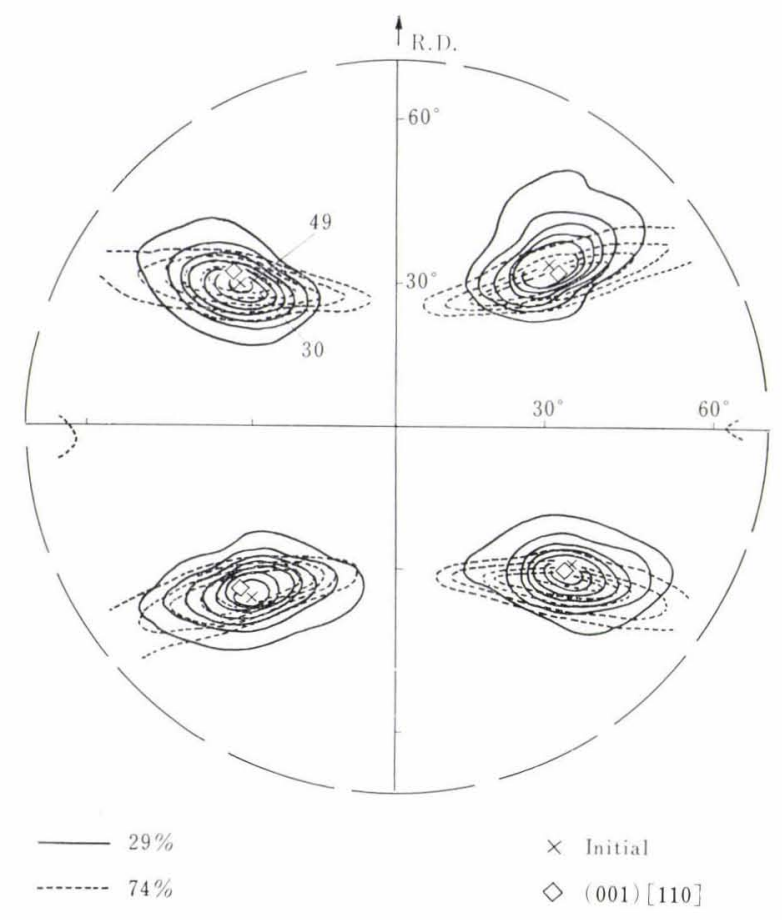

Fig. 9. (110) pole figure of (001)[110] crystal, SI-37, rolled to 29 and $74 \%$ reduction stage. Fig. 8 is the slip bands observed on the front and rolling surfaces in the crystal rolled to $29 \%$ reduction. Referring to the schematic orientation diagrams, it is found that the slip during rolling occurred on four $\{110\}$ planes, $A, B, C, D$, and two $\{112\}$ planes, $b, h$. The deformation texture of the crystal remains in near the initial orientation as shown in Fig. 9. Strictly speaking, the peak positions of it approach the poles of the ideal orientation (001) [110]. Namely, the (001)[110] orientation is stable for rolling ${ }^{4}$, though the texture spreads around the rolling direction with reduction, while that around the transverse direction is rather weak at lower reduction and more so at higher reduction.

The rolling textures of the $(015)[100]$ crystal, SI-

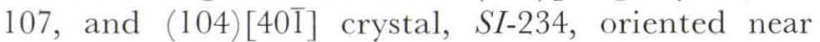
(001) [100] are shown in the (110) pole figures of Figs. 10 and 11, respectively. In the (015)[100] crystal, the deformation texture splits into two components that are related to each other by rotation around the [001] axis of the initial orientation and the two components approach the end orientation $\{115\}\langle 110\rangle$.

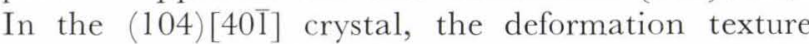
rotated to the $(001)[100]$ orientation around the transverse direction [0 $\overline{1} 0]$ at early stage of rolling and then splitted into two components by rotation around 

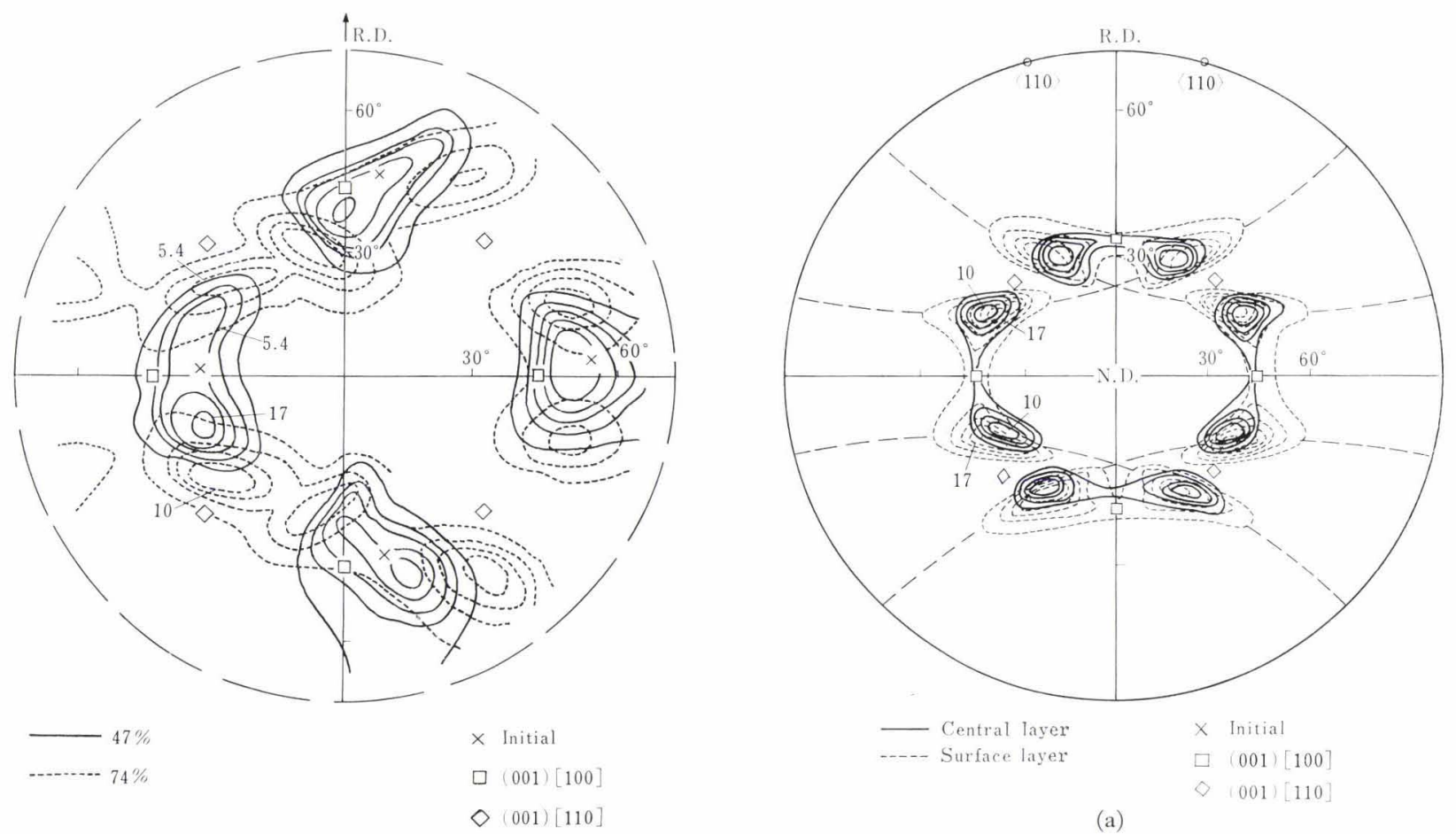

Fig. 10. (110) pole figure of (015)[100] crystal, $S I-107$, rolled to 47 and $74 \%$ reduction

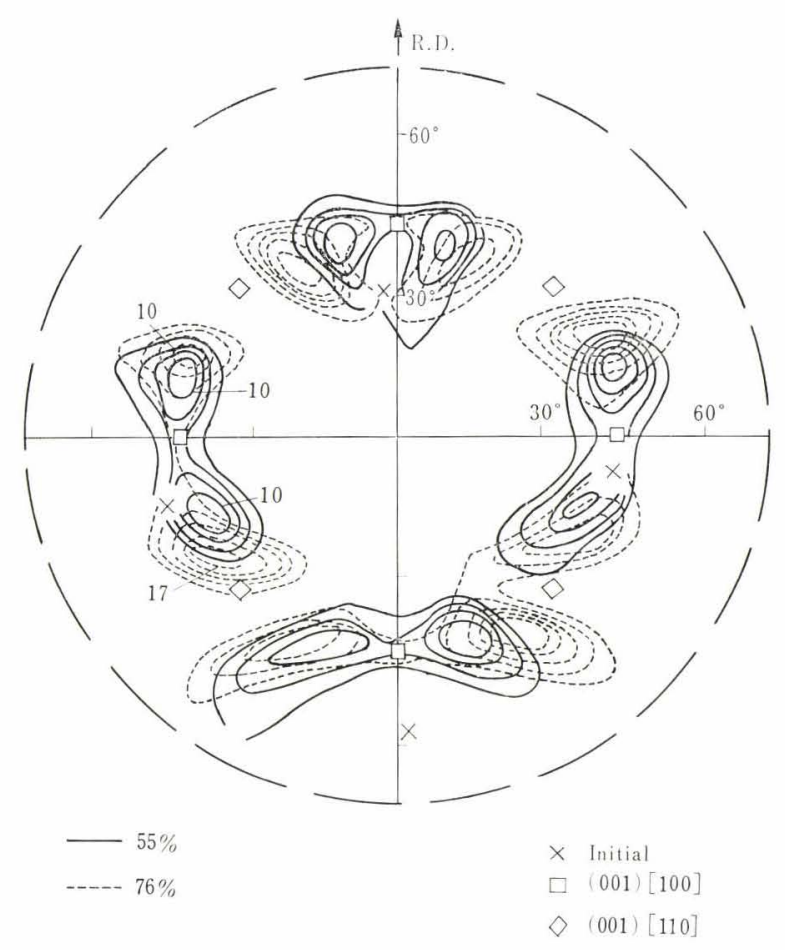

Fig. 11. (110) pole figure of (104)[401] crystal, SI-234, rolled to 55 and $76 \%$ reduction

the [001] axis, both of which approach the stable end orientation along the same path as those of the (001) [100] crystal, in a manner similar to the result of Walter and Hibbard. ${ }^{5)}$

The metallographic structure of these rolled crystals was composed of bands elongated nearly parallel to the rolling direction, which was similar to the banded structure of the (001) [100] crystal in Photo 1.

In all the crystals included in this series, $S I$, it has

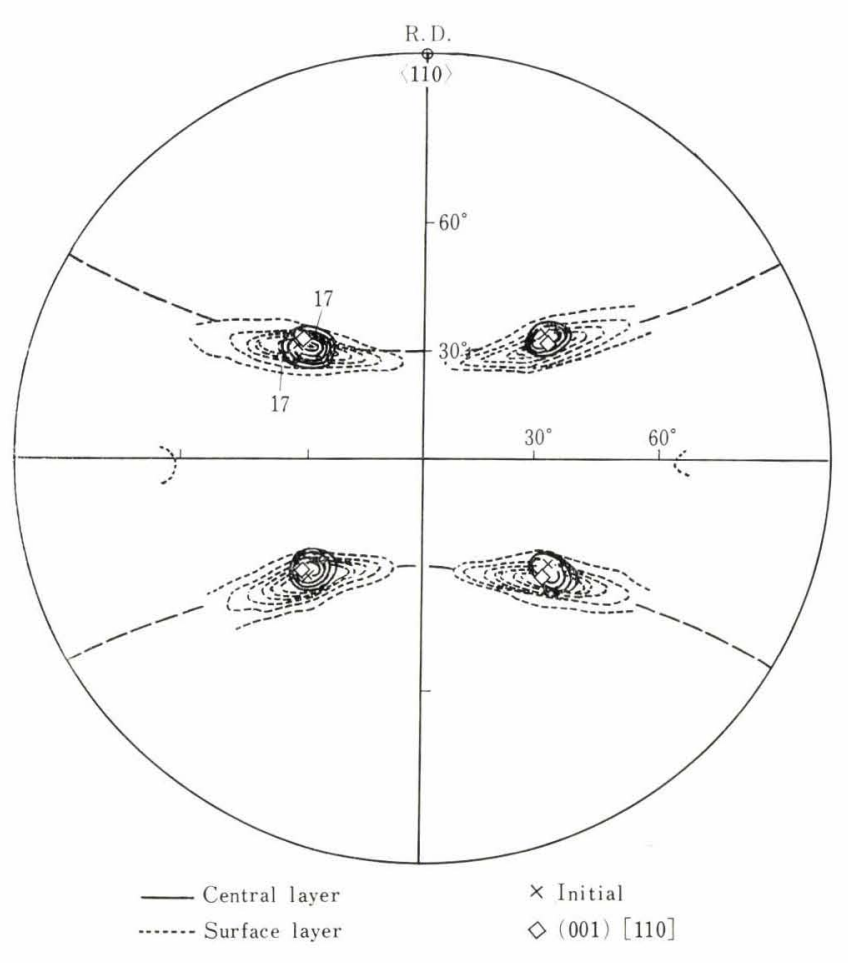

(b)

Fig. 12. (110) pole figure of central surface layers. Dashed lines are $60^{\circ}$ small circles around $\langle 110\rangle$ poles indicated in the figures.

(a) (001)[100] crystal, $S I-77$, rolled to $75 \%$ reduction

(b) (001)[110] crystal, $S I-37$, rolled to $74 \%$ reduction

been found that the crystals rotate to one or two symmetrical components oriented between $\{001\}\langle 100\rangle$ and $\{001\}\langle 110\rangle$, and then approach the stable end orientation $\{001\}\langle 110\rangle$ with increasing reduction. In the crystals in which two components are developed, the rolling structure was composed of bands elongated in the rolling direction, and the orientations of 
the alternate band being the same, corresponding to either component of the texture, in agreement with other works. ${ }^{14), 16)}$

The rolling texture in surface layer showed wider spread than that in central layer in all crystals, as seen in Fig. 12 (a) and (b) (see p. 299). The figures (a) and

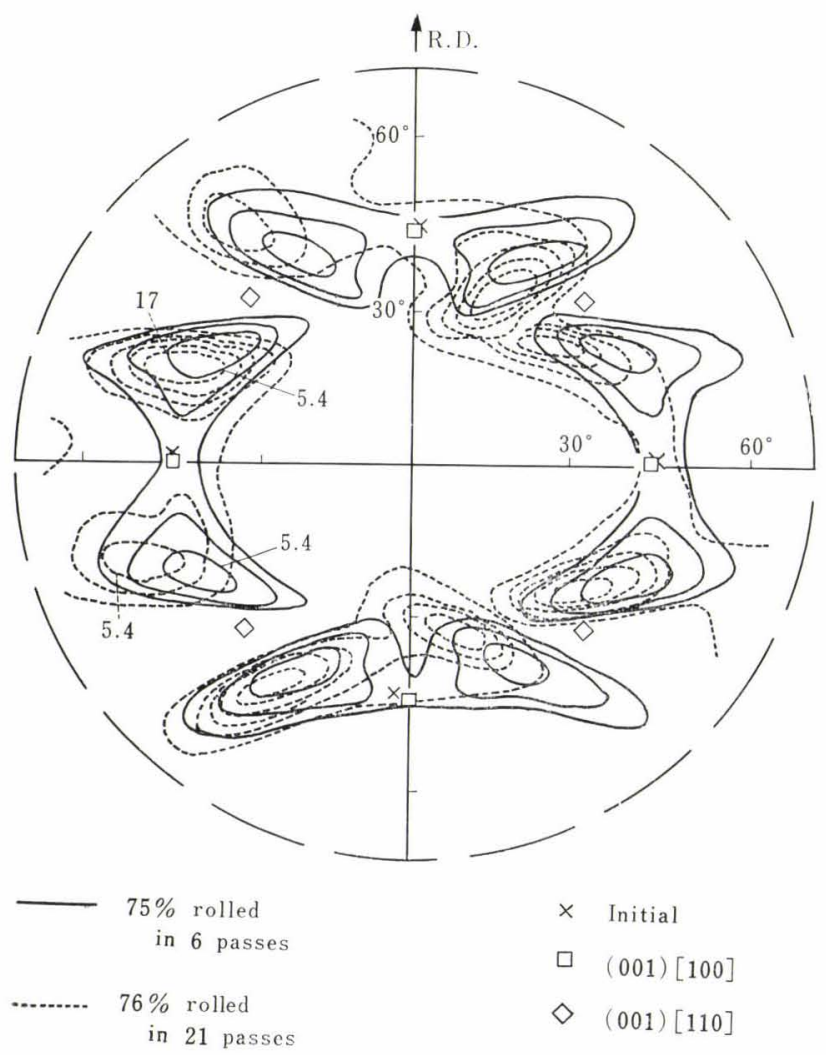

Fig. 13. (110) pole figure of a (001)[100] crystal rolled to $75 \%$ reduction in 6 passes and the same $(001)$ [100] crystal rolled to $76 \%$ reduction in 21 passes (b) are the (110) pole figures in surface and central layers of the (001) [100] crystal rolled to $75 \%$ reduction and of the (001)[110] crystals rolled to $74 \%$ reduction, respectively. In all the crystals of $S I$, the surface textures spread around a $\langle 110\rangle$ direction that is nearest to the rolling direction. However, the general feature such as the peak position did not differ so much from each other.

Moreover, the rolling texture depended on the number of passes to give the same reduction in thickness. Fig. 13 shows the difference in the pole figures of the same (001)[100] crystal rolled to $75 \%$ in 6 passes and $76 \%$ in 21 passes. The texture rolled at the lower reduction per pass spread more than that at the higher reduction per pass and deviates from the symmetrical texture of the latter. All the other crystals were rolled at the high reduction per pass in the present experiment.

\section{Second Series, SII}

At first, crystals with (001)[100] orientation, SII108, SII-23, SII-31, SII-29, SII-33, and SII-37 were rolled to various reductions of 8.2 to $86 \%$, see Table 2. The average rolling pressure during each pass increased with increasing reduction at higher rate than that in (001)[100] crystal, as shown in Fig. 14 (a). In these graphs, the values beyond the dot-and-dashed lines are the apparent ones where the two working rolls made direct contact due to elastic deformation. The change of Vickers microhardness with increasing reduction is also presented in the figure which shows continuous increase of hardness to higher reductions. The slip pattern of the $(011)[100]$ crystal, SII-108, rolled to $25 \%$ reduction, is presented in Fig. 15. The operating slip planes are found to be mainly $\{110\}, C, E$, and $\{112\}, f$, the planes which contain a

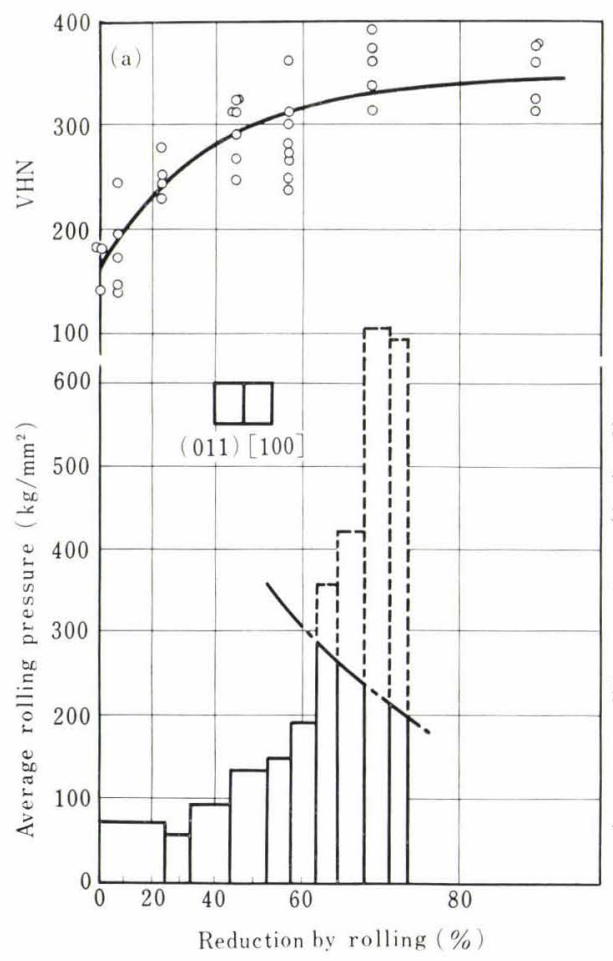

Reduction by rolling ( $\%$

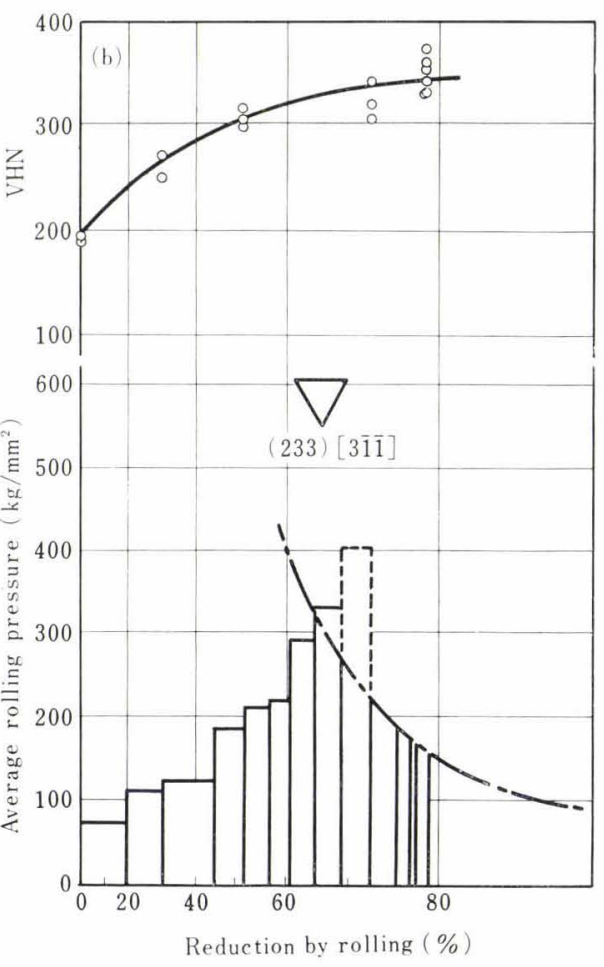

Fig. 14 .

Changes of average rolling pressure and Vickers micro-hardness with reduction. The rolling pressure values beyond dot-and-dashed lines are only apparent as two working rolls made a direct contact.

(a) .(011)[100] crystal, $S I I-108$ (b) (233) [311] crystal, SII-104 

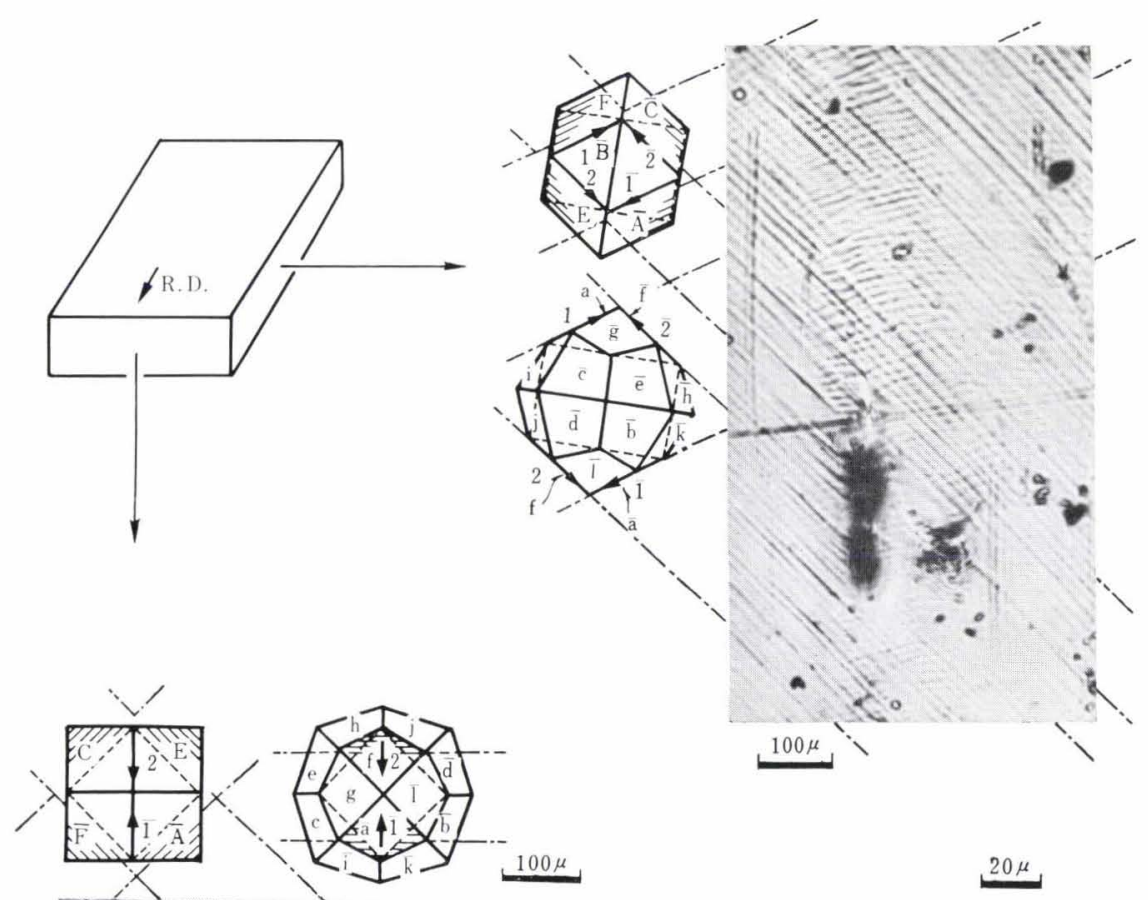

$100 \mu$

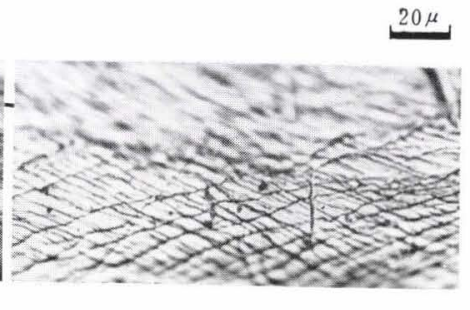

Fig. 15.

Slip patterns on front and side surfaces of (011)[100] crystal, SII-108, rolled to $25 \%$ reduction

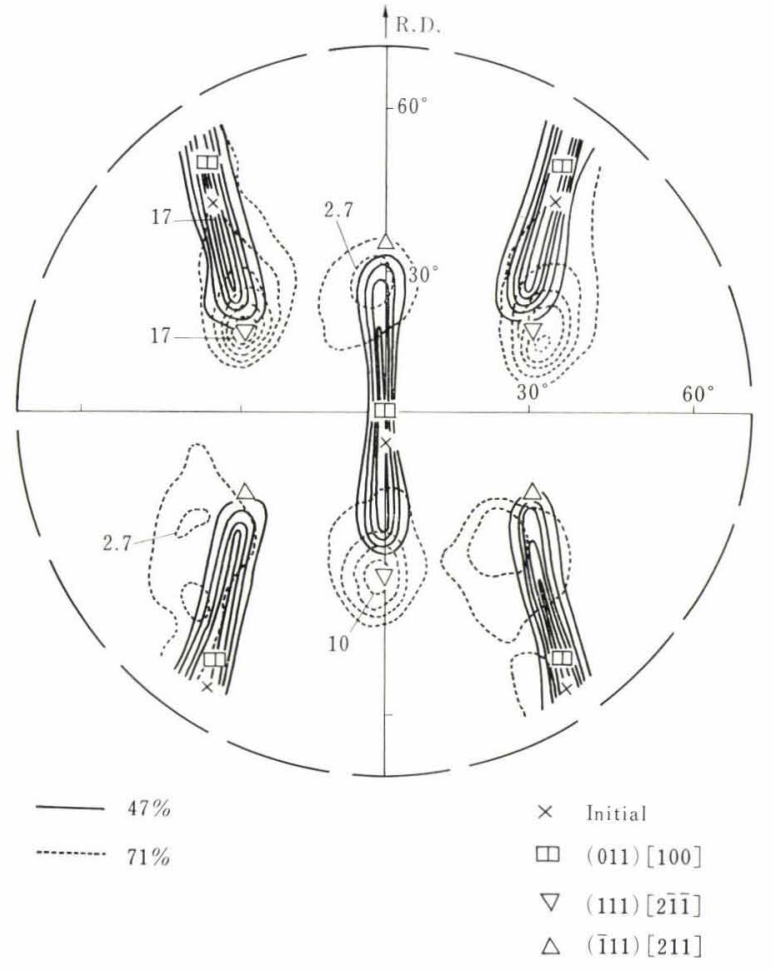

Fig. 16. (110) pole figure of (011)[100] crystal, SII-23, rolled 47 and $71 \%$ reduction, rectangles, downward and upward triangles indicate $\{110\}$ poles of (011)[100], (111)[211] and (111)[211], respectively

common $\langle 111\rangle$ slip direction, 2. In other regions, traces of $\{110\}, A, F$, and $\{112\}, a$, the planes which contain another common $\langle 111\rangle$, 1 , were observed.

The rolling texture of the crystal with (011)[100] orientation, $S I I-23$, rolled to 47 and $71 \%$ reduction are shown in the $(110)$ pole figures of Fig. 16. The texture spreads in the longitudinal direction at early stage $^{2), 23)}$ and splits into two symmetrical components as the rotation occurred in both directions around the transverse direction [0]1]. The two com-

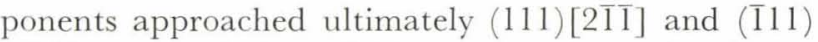
[211], respectively, which are stable end crientations. ${ }^{3), 5), 7)}$ The process by which the stable end orientations are established in the rolling texture is clearly shown in Fig. 17, which presents the change of longitudinal profile of central (110) pole distributions in Fig. 16. It is attributed mainly to the deviation of the initial orientation from ideal (011)[100] that the one component is stronger than the other in Fig. 17. The rotation angle of pole density maximum of each component increases with increasing reduction up to $50 \%$, then approaches the $35^{\circ}$ angle asymptotically, as shown in Fig. 18, which will be discussed in a later section.

The topographic distribution of the two components of the rolling texture was not so regular as the banded structure of the (001) [100] crystal, an example is shown in Photo 3 (a) and (b), which are macro-etched and micro-etch pit patterns of the $32 \%$ rolled (011) [100] crystal, respectively. Irregularly distributed bright regions in (a) were found to correspond to the initial orientation (011)[100]. The straight bright bands in (a) had nearly $\{100\}\langle 011\rangle$ orientation as 


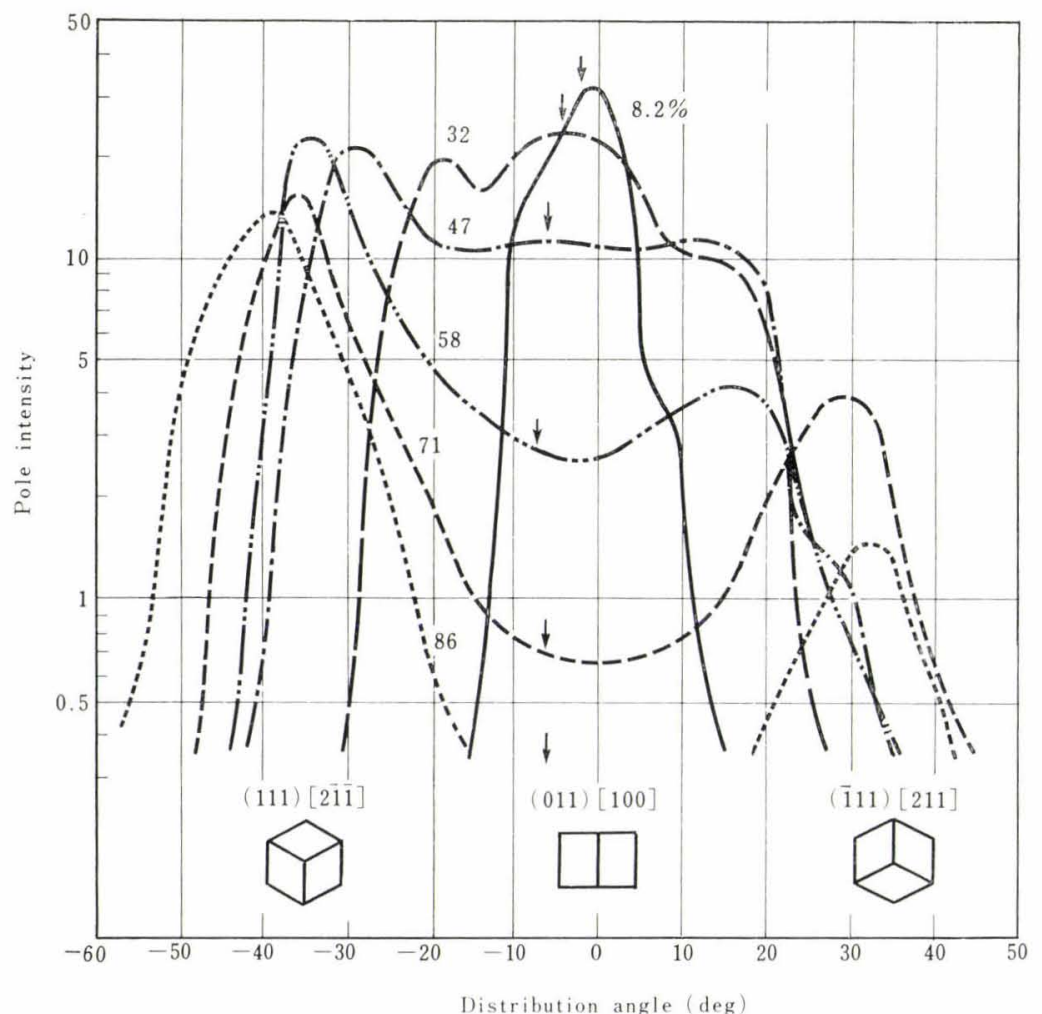

Fig. 17.

Longitudinal profiles of central (110) pole density at various stages of rolling in (011)[100] crystals. Small arrows indicate initial orientations.

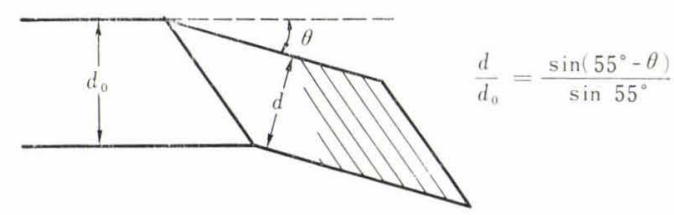

$$
X=\frac{d_{0}-d}{d_{0}}
$$

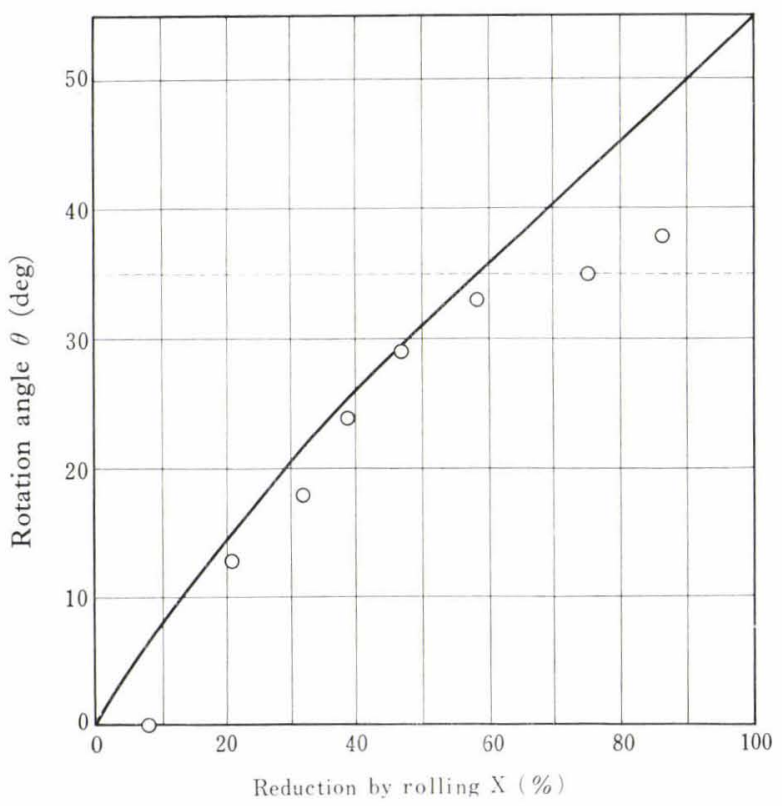

Fig. 18. Rotation angle of rolling texture around transverse direction as a function of rolling reduction in (011)[100] crystal, together with calculated curve by a simple geometrical relation

shown in (b), and corresponded to the twin bands formed in the first reduction of rolling, as pointed out by Dunn. ${ }^{3)}$ On the other hand, the dark regions, corresponded to either of the two components with $\{111\}$ planes being nearly parallel to the specimen surface. Each region of the two components had neither difinite shape nor size. The size widely varied mainly according to the initial orientation and also to the amount of the first reduction.

In a crystal with (233)[311], SII-104, oriented be-

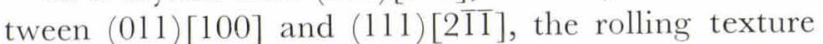
spreads in the longitudinal direction and the mean orientation rotated towards the (111)[2]1] orientation at lower reduction as shown in the (110) pole figures of Fig. 19. At higher reduction, the texture spreads more around the transverse direction. The micro-etch pit pattern on the side surface at the $73 \%$ reduction is shown in Photo 4 . The micro-etch pits indicate that the orientation changes discretely from one region to the other; the regions are occasionally connected with twin bands indicated by $\mathrm{T}$.

The rolling texture of the (322)[453] crystal, SII304 , oriented between (111)[211] and (211)[111] is shown in Fig. 20. The texture spreads around the

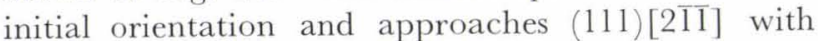

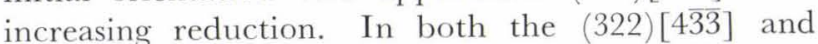
(233) [3TI] crystals, oriented near the (111)[2TI], the mean orientation approached the (111)[2]1] orientation at early stage of rolling, then the two crystals

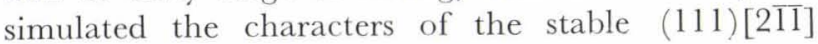
crystal at later stage.

The average rolling pressure as well as the Vickers microhardness of (111) [211] crystals increased rapidly from the outset as the reduction increased, as shown in Fig. 14 (b) by an exemplary case where the initial orientation was near (111)[2]1]. Fig. 21 shows the (110) pole figure of the crystal with (111)[2]1] 

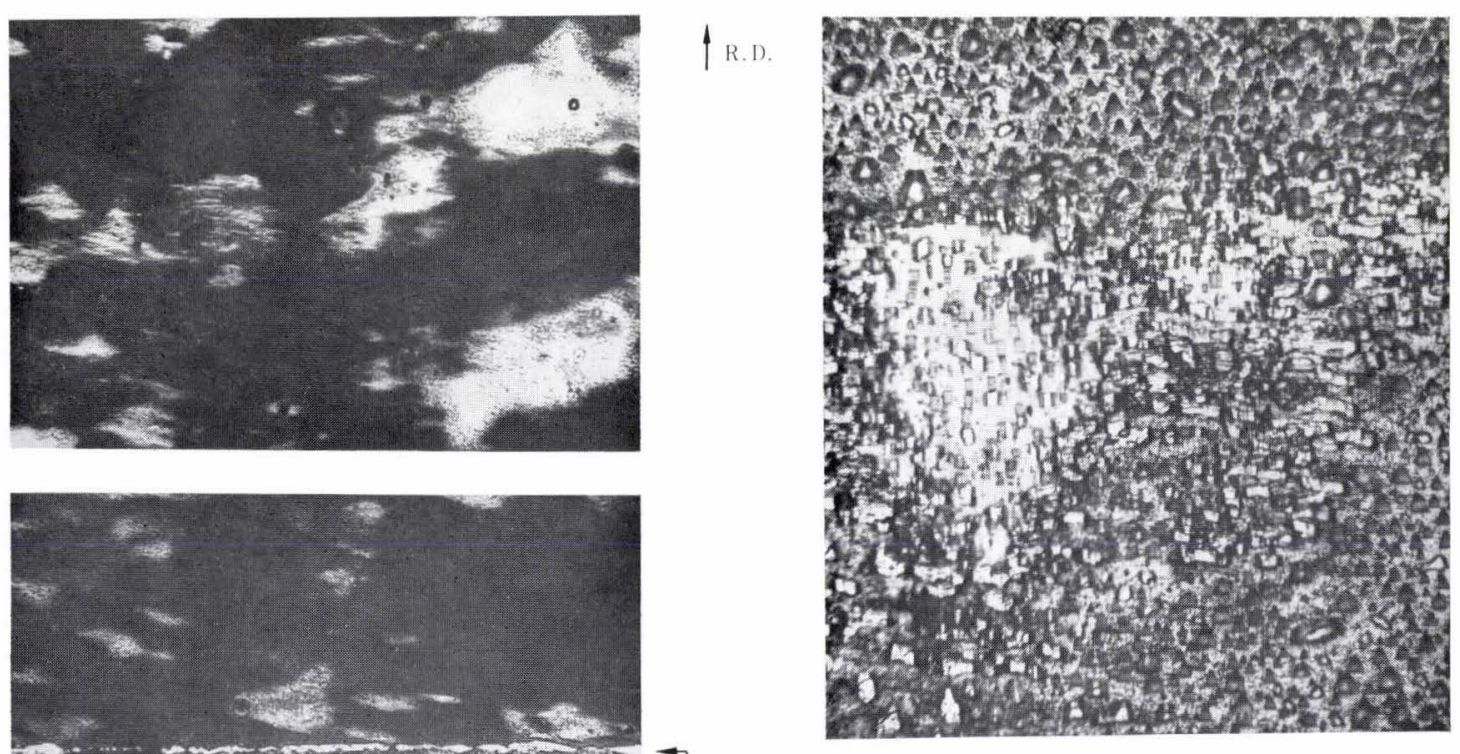

Photo 3 .

Metallographic structure of rolling surface of
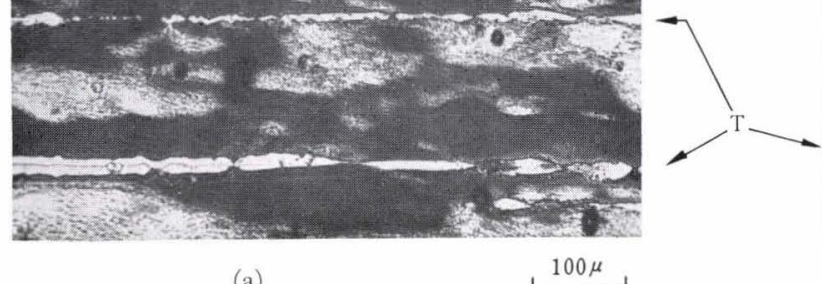

(a)

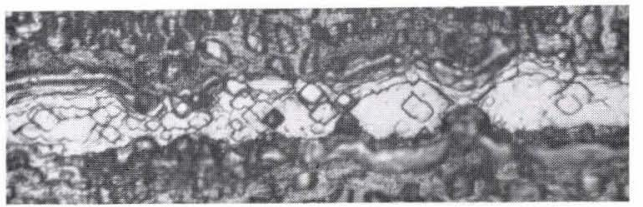

(011)[100] crystal, $S I I-33$, rolled to

$32 \%$ reduction

(a) Macro-etched structure

(b)

$10 \mu$

(b) Micro-etch pit pattern
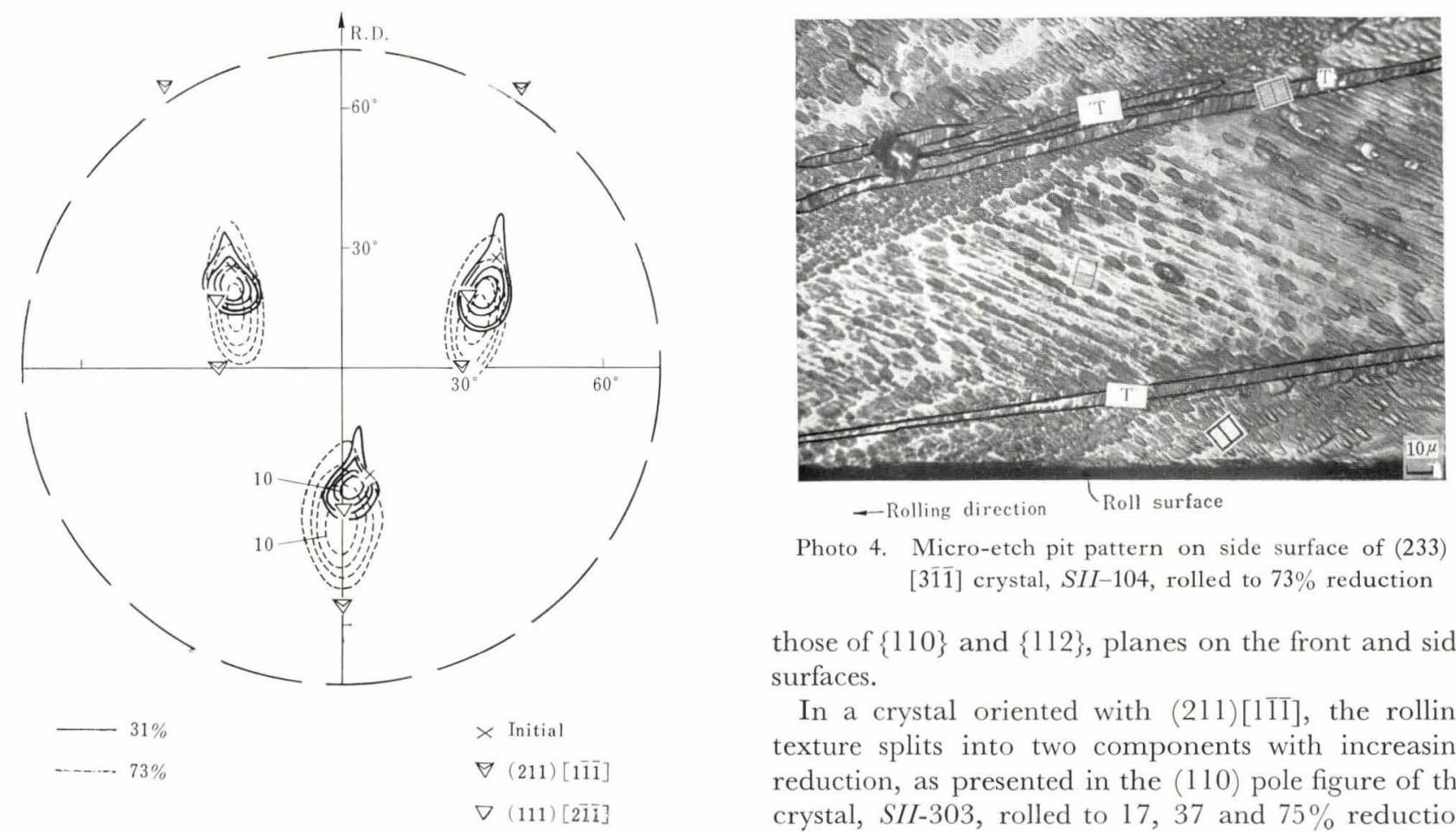

Photo 4. Micro-etch pit pattern on side surface of (233) [311]] crystal, $S I I-104$, rolled to $73 \%$ reduction

those of $\{110\}$ and $\{112\}$, planes on the front and side surfaces.

In a crystal oriented with (211)[1i] , the rolling texture splits into two components with increasing reduction, as presented in the (110) pole figure of the crystal, SII-303, rolled to 17,37 and $75 \%$ reduction in Fig. 23. One component rotates to (111)[211] while the other to $(100)[011]$ both around the transverse direction [011]. The observed values of rotation angle in each component as a function of reduction are presented in Fig. 24. The (111)[211] side component almost reaches the final orientation (111) [211] at about $40 \%$ reduction and maintains the orientation through higher reductions, while the (100) [0II] side component reaches the final orientation (100) $[0 \overline{11}]$ at about $60 \%$ reduction.

It is interesting to note the characteristic distribu- 
tion of the two components across the thickness in the rolled (211)[1]1] crystal. Their distribution was examined using the pole figure and metallographic techniques by removing layers successively from the surface. The distribution was neither uniform nor symmetrical with respect to the central section as shown in Fig. 25. The (100)[011] component is stronger near the surface in agreement with the result of $\mathrm{Hu}$ and Cline. ${ }^{24)}$ However, the (111)[2]1] component is stronger in the lower half region than in the upper, in contrast with their result. ${ }^{24)}$ These features will be discussed later in consideration of operating

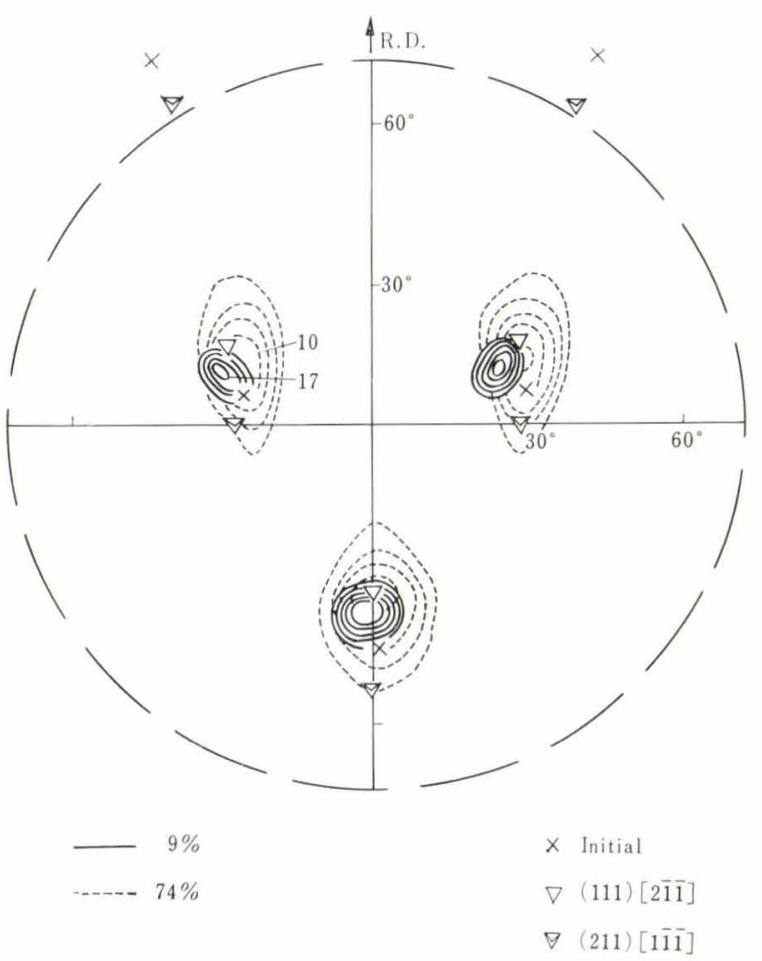

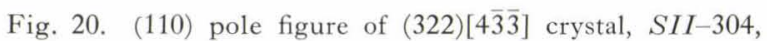
rolled to 9 and $74 \%$ reduction

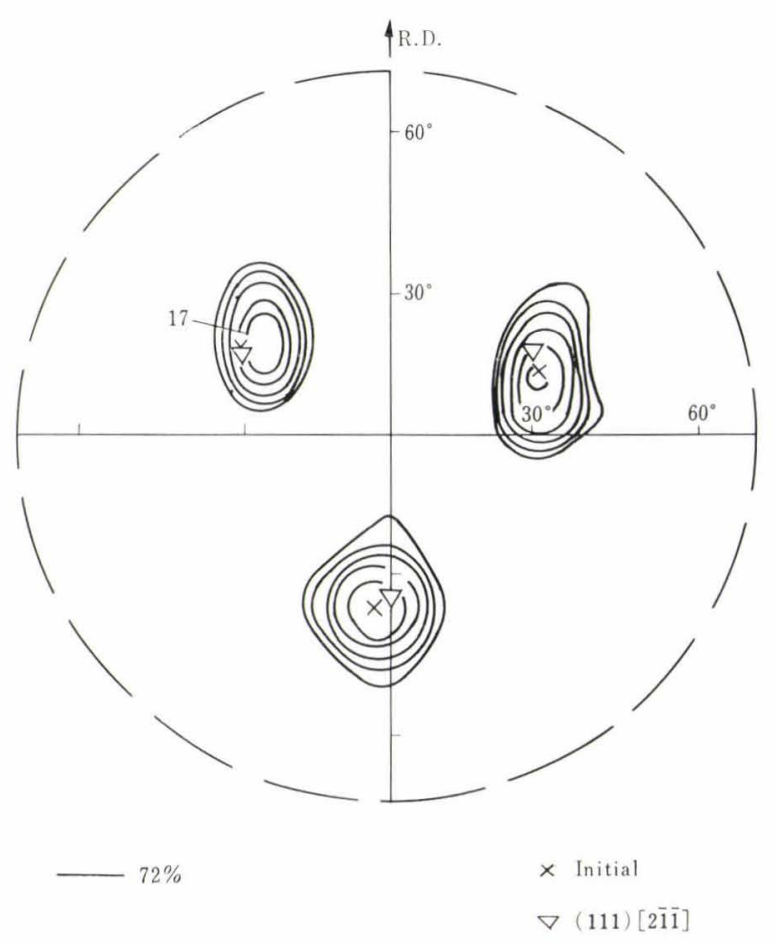

Fig. 21. (110) pole figure of (111)[21̄1] crystal, SII-302, rolled to $72 \%$ reduction

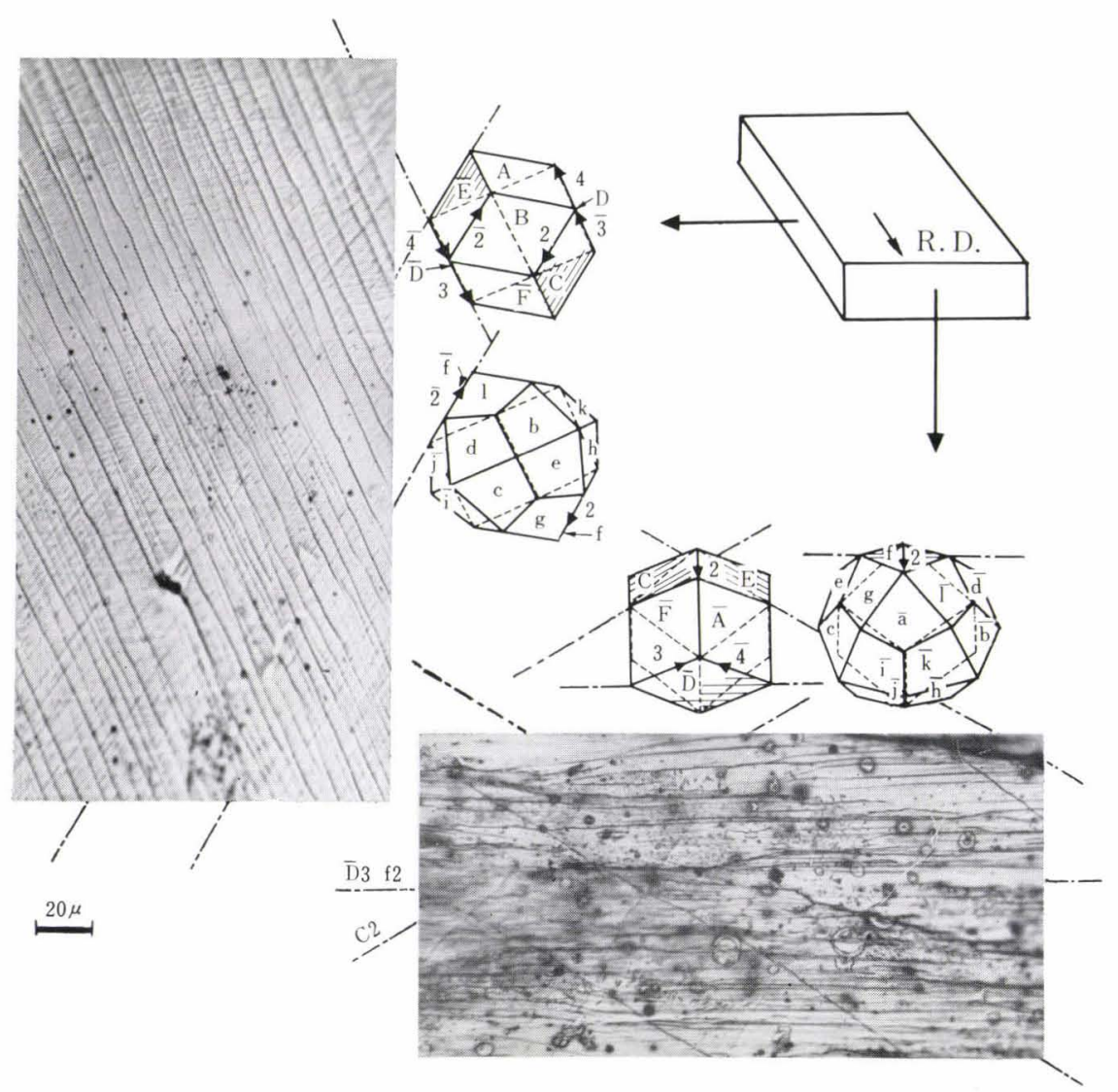

Fig. 22.

Slip patterns on front and side surfaces of (111)[211] crystal, $S I I-302$, rolled to $9 \%$ reduction 


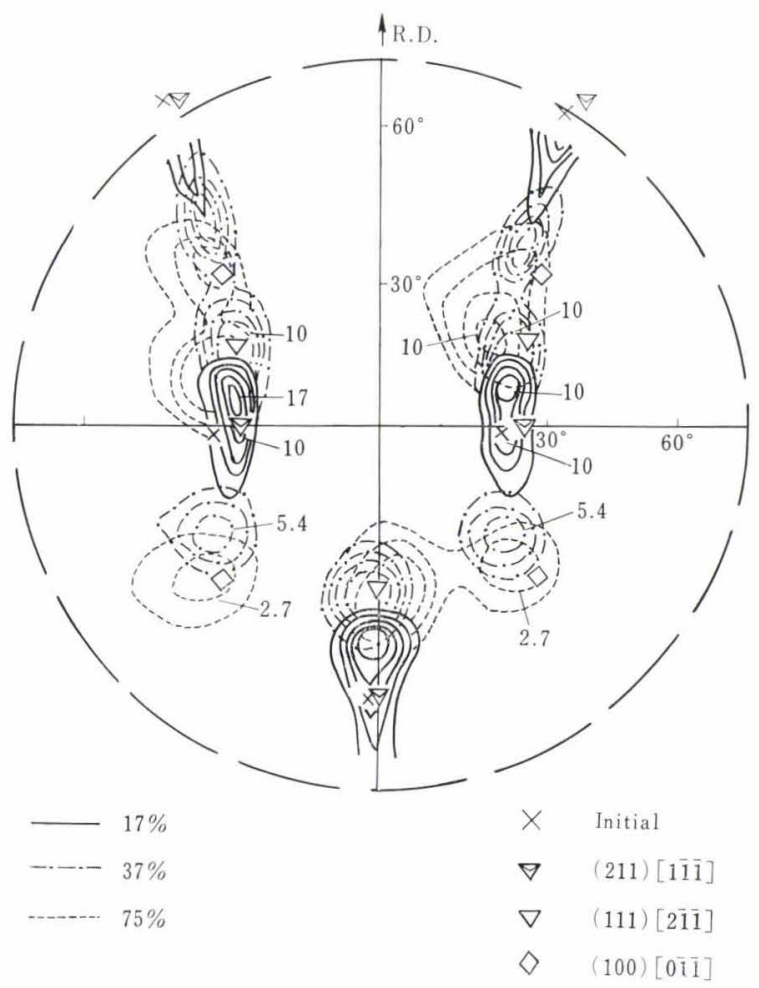

Fig. 23. (110) pole figure of (211)[111] crystal, SII-303, rolled to 17,37 and $75 \%$ reduction

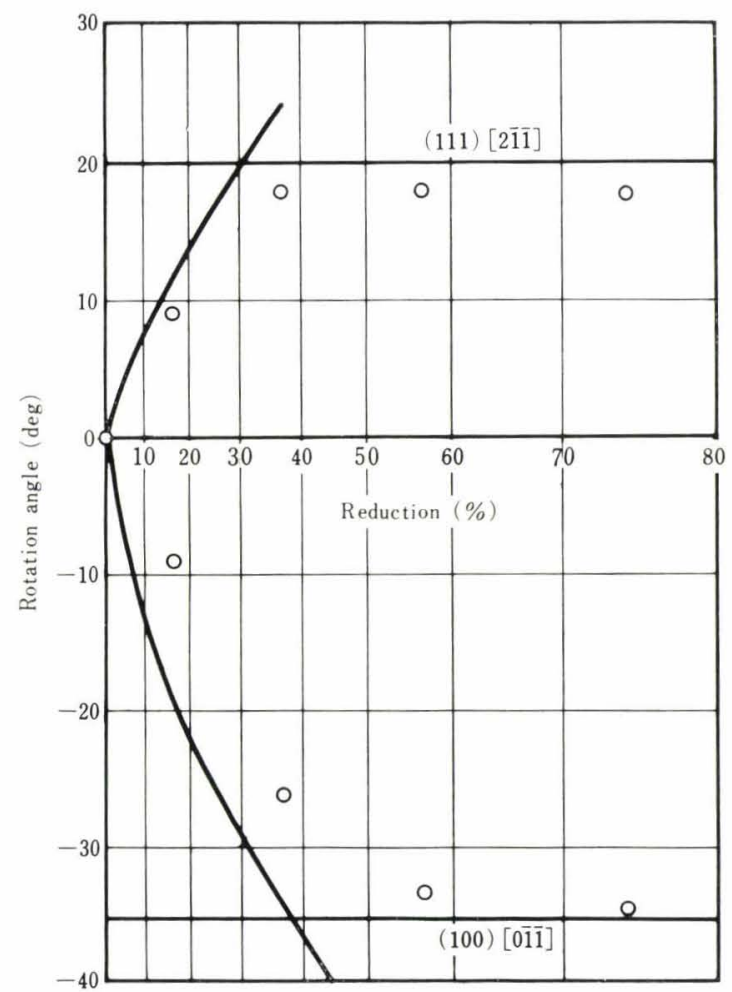

Fig. 24. Rotation angle of two components in rolling texture of (211)[111] crystal as a function of rolling reduction, together with calculated curves by simple geometrical relations

slip systems in this crystal. Slip patterns on the three surfaces of the (211)[1Ti] crystal rolled to $17 \%$ reduction are shown in Fig. 26. The slip planes operated during the rolling are found to be $\{110\}, A, D, F$, and

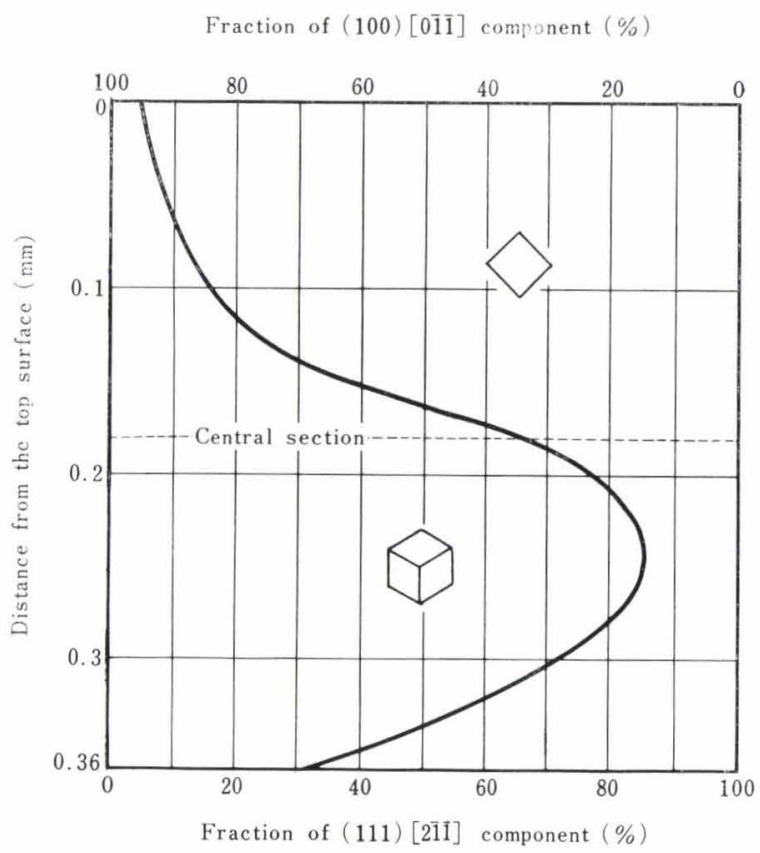

Fig. 25. Distribution of component (111)[211] and (100)

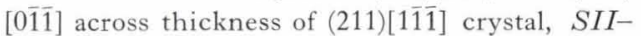
303 , rolled to $57 \%$ reduction

$\{112\}$, a from the observed slip traces.

In the crystal with (511)[255], SII-112, oriented between (211)[111] and (100)[0]1], the mean orientation rotates to the orientation (100)[0]1] with increasing reduction as shown in the (110) pole figure of Fig. 27.

In addition, the rolling texture of the (025) [100] crystal, SII-111, rolled to 48 and $77 \%$ reduction, oriented near the typical orientation (011)[100], is shown in the (110) pole figures of Fig. 28. At early stage, the rolling texture spreads and then splits into two symmetrical components, both of which are related to the initial orientation by rotation around the [01] axis in the figure. At higher reduction, each com-

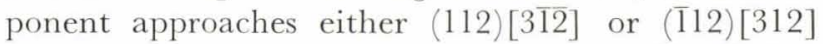
orientation. Similar textures were reported by Walter and Hibbard ${ }^{5)}$, Dunn ${ }^{11)}$ and $\mathrm{Hu}^{7}$. Metallographic structure of the as-rolled crystal showed a banded structure similar to that of the (001)[100] crystal though the boundary planes were somewhat more irregular and inclined, as shown in Photo 5. The increase of rolling pressure with increasing reduction was rapid as in the (011)[100] crystal.

Thus, it was found that the cold rolled texture of all the crystals oriented between (011)[100] and (211) [11i] is gradually rotated with reduction to the stable end orientation (111)[2II] around the transverse [011] direction, which is maintained during rolling. Whereas, the cold rolled texture of all crys-

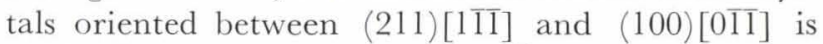
gradually rotated to the $(100)[0 \overline{1}]$ stable end orientation in the same way described above. The rolling texture of two crystals oriented in ideal (011) [100] and (211)[111] splitted into two components each of which approaches to the respective stable end orien- 


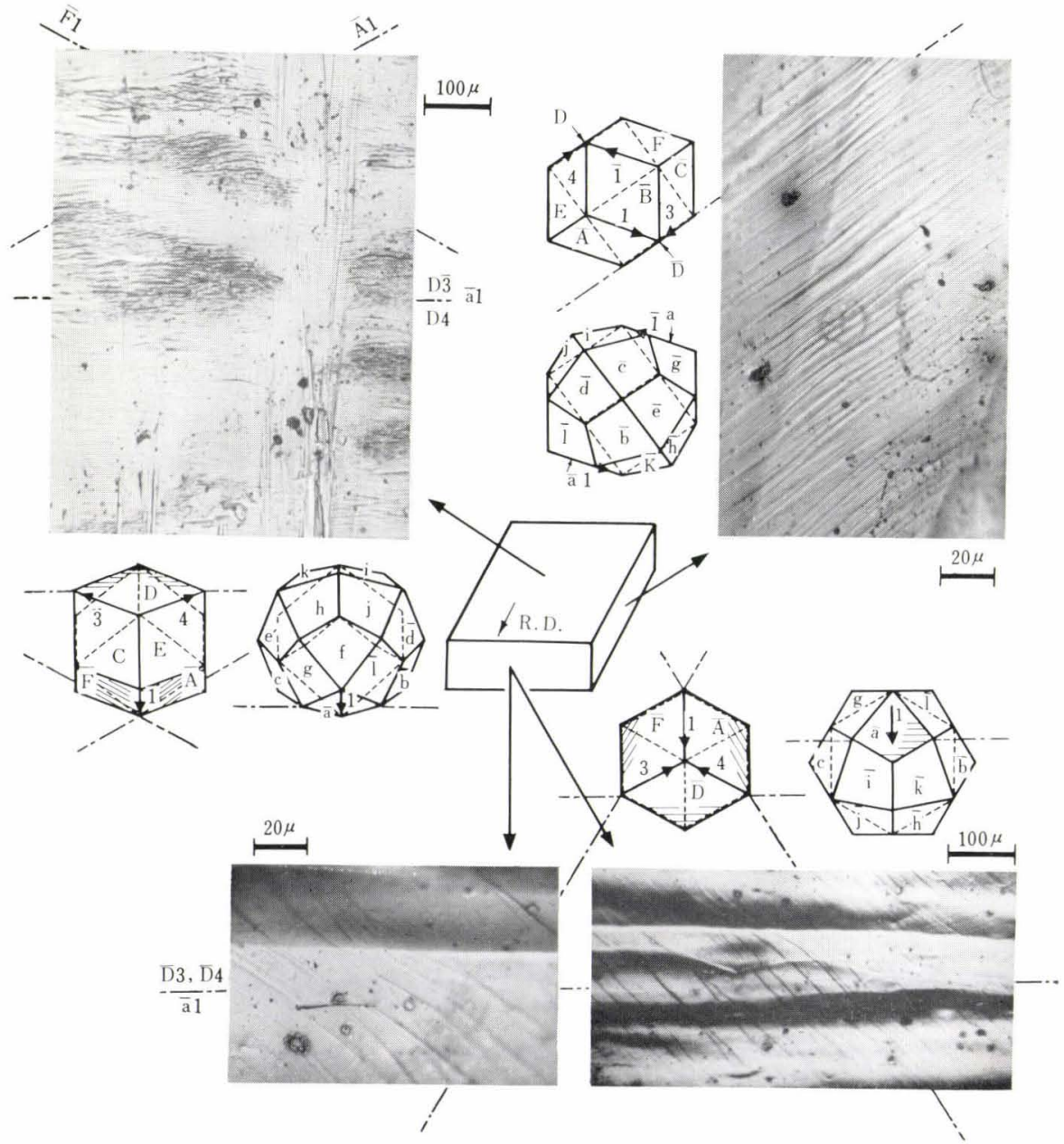

Fig. 26.

Slip patterns on front, side and rolling surfaces of (211)[111] crystal, SII-303, rolled to $17 \%$ reduction

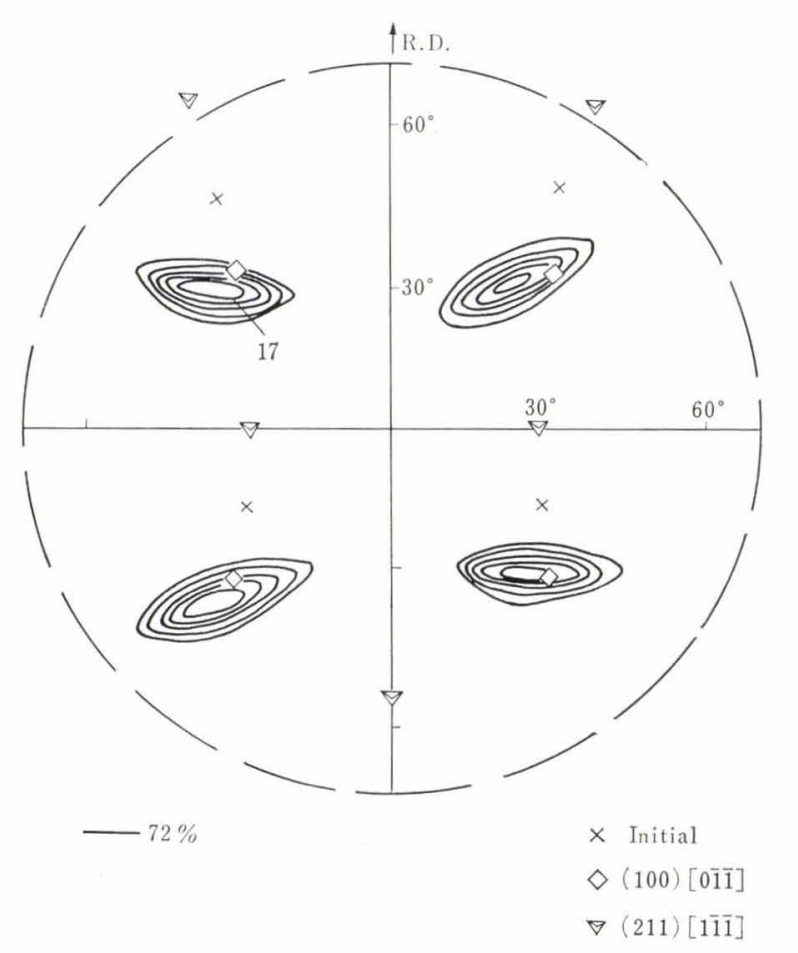

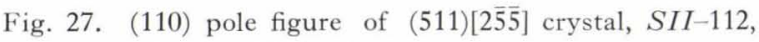
rolled to $72 \%$ reduction

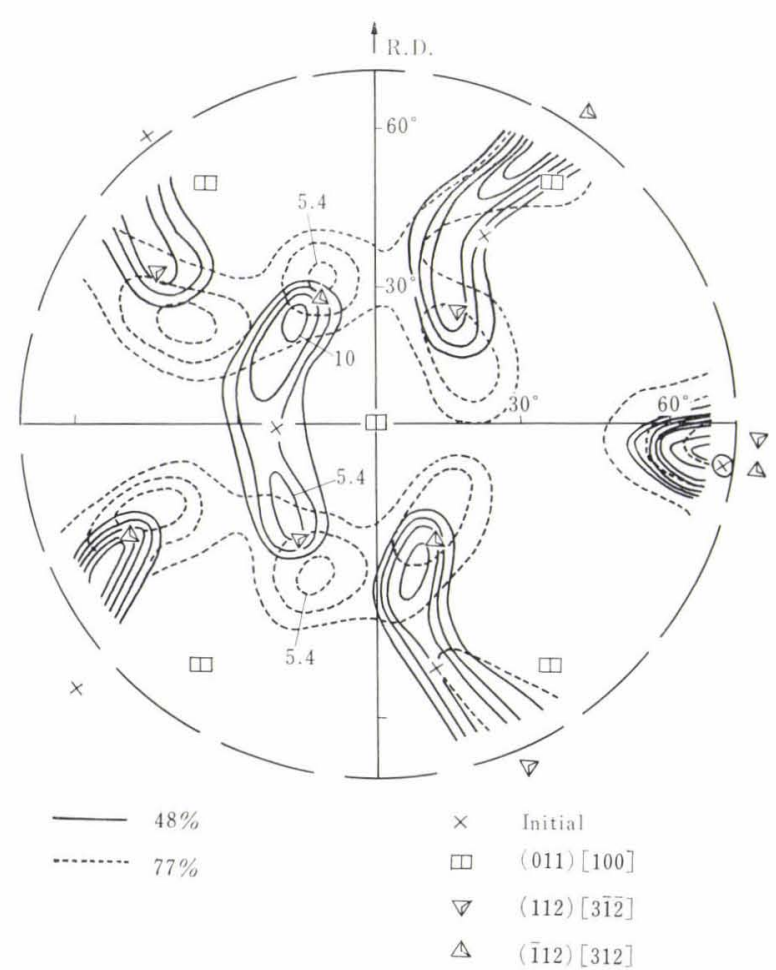

Fig. 28. (110) pole figure of (025)[100] crystal, SII-111, rolled to 48 and $77 \%$ reduction 


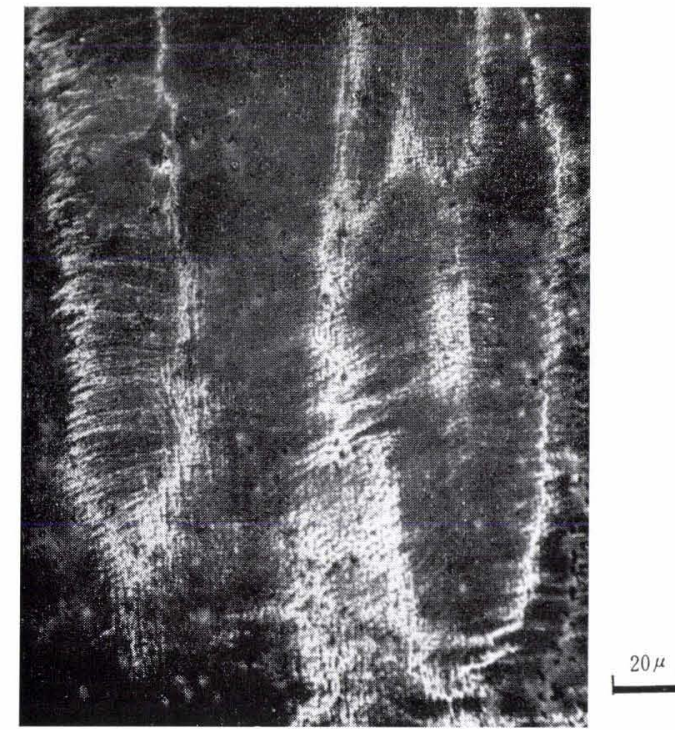

(a) Rolling surface

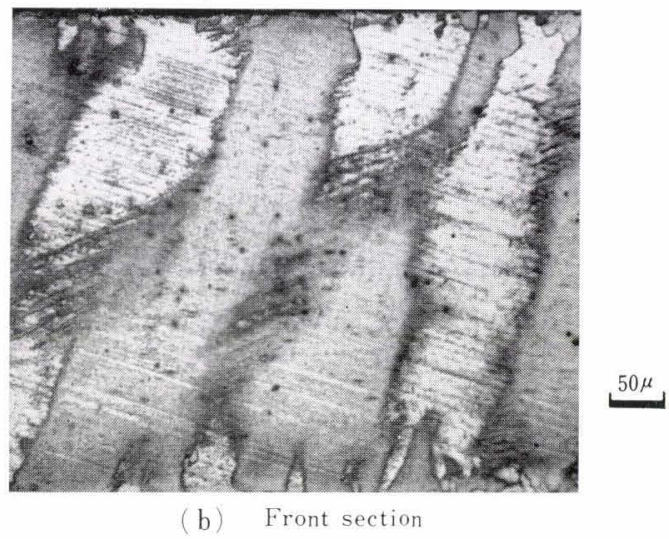

Photo 5. Macro-etched structures on rolling and front surfaces of (025)[100] crystal, SII-111, rolled to $48 \%$ reduction

tations. These features are in qualitative agreement with the reported results. ${ }^{3), 4), 7), 24 \text { ) }}$

\section{Third Series, SIII}

The $(100)[0 \overline{1} \overline{1}]$ crystal belongs to all three series and is a typical stable end orientation as described in the first series.

Fig. 29 is the (110) pole figure of the crystal initially oriented near $(2 \overline{1} 1)[0 \overline{1}]$, SIII-215 rolled to $78 \%$ reduction. The initial orientation is almost maintained, but tends to approach the ideal orientation

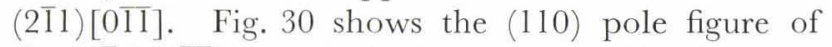
the $(1 \overline{1} 1)[0 \overline{11}]$ crystal, $S I I I-210$ rolled to $74 \%$ reduction. The rolling texture also tends to approach the $(2 \overline{1} 1)[0 \overline{1}]$ orientation. The rolling texture hardly

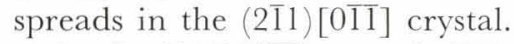

In the (101)[121] crystal, SIII-17, oriented between two symmetrical $\{211\}\langle 011\rangle$ orientations, the rolling texture splits into the two $\{211\}\langle 011\rangle$ components those are related to each other by rotation around the common transverse direction [1]1], as shown in Fig. 31. It is supposed to be due to the deviation of the initial orientation from the ideal orientation (101) [121] that the two components deviated from the ideal orientations $\{211\}\langle 011\rangle$.

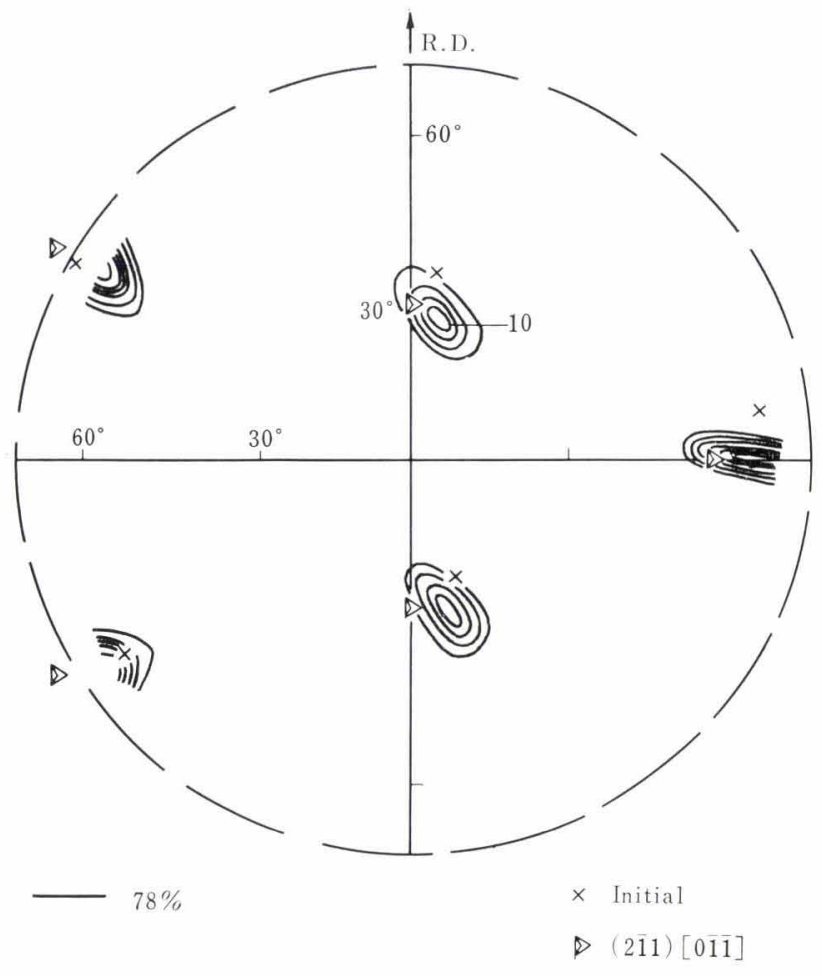

Fig. 29. (110) pole figure of (211)[011] crystal, SIII-215, rolled to $78 \%$ reduction

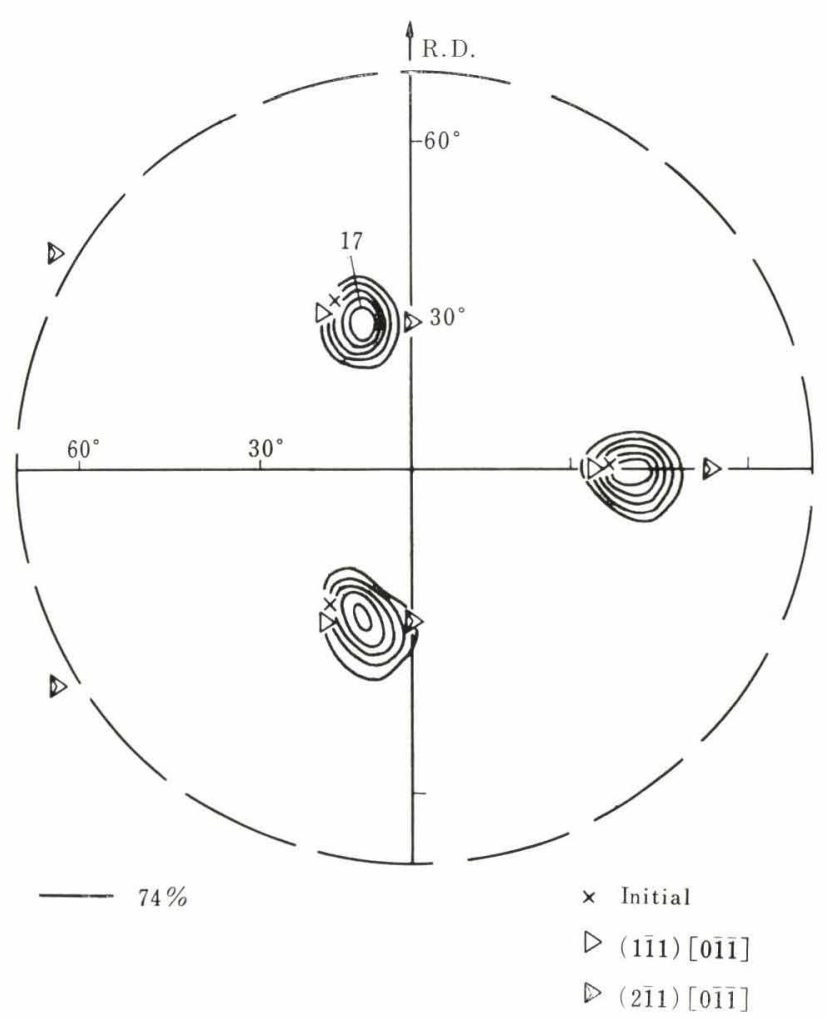

Fig. 30. (110) pole figure of (1111)[011] crystal, SIII-210 rolled to $74 \%$ reduction

Another crystal in this series with a typical orientation (011)[011], SIII-108, was rolled to 6 and $71 \%$ reduction. The slip patterns were observed after $6 \%$ reduction, as shown in Fig. 32. The operating slip planes are found to be mainly $\{110\}, A, C, E, F$, from 


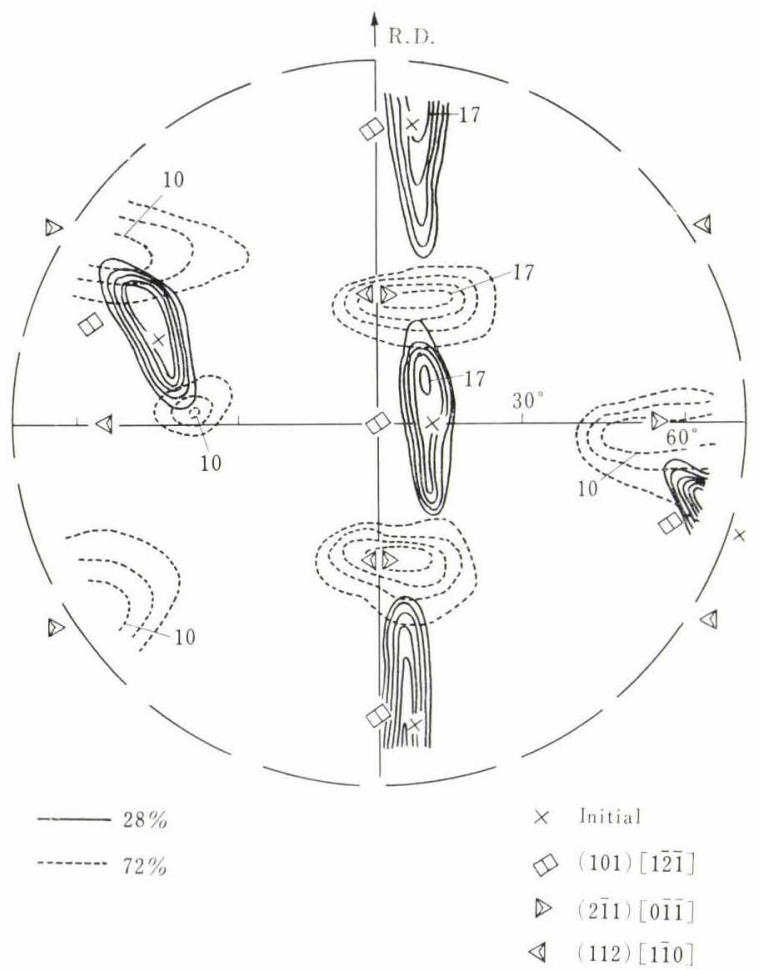

Fig. 31. (110) pole figure of (101)[211] crystal, $S H I-17$, rolled to 28 and $72 \%$ reduction the observed slip traces. The slip bands in this crystal are coarse and sharp. Dark broad bands observed on the front surface corresponded to large steps. It is worth to note that the width of this crystal increased by about $2.8 \%$ while the length only by $3.4 \%$ after $6 \%$ reduction in thickness, suggesting the difficulty of rolling deformation. Actually, the average rolling pressure during the first $6 \%$ reduction was as great as $190 \mathrm{~kg} / \mathrm{mm}^{2}$. The rolling texture spreads around the initial orientation at early stage, becoming progressively diffuses with increasing reduction, as shown in Fig. 33. At $71 \%$ reduction, the main texture tilts from the initial orientation around the rolling direc-

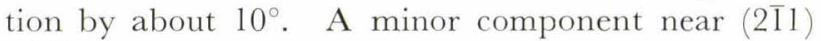

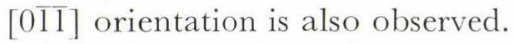

In addition, the rolling texture of the $(0 \overline{3} 5)[0 \overline{5} 3]$

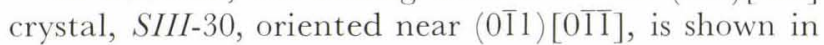
the (110) pole figures of Fig. 34. The texture spreads around the initial orientation in early stage, then

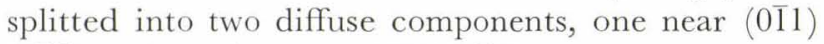

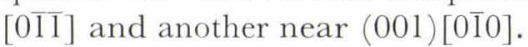

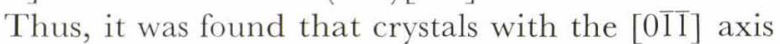
parallel to the rolling direction, except $(0 \overline{\mathrm{I}} 1)[0 \overline{\mathrm{l}}]$ and its neighbours, almost maintain their initial orientations during rolling in agreement with the reported result. ${ }^{4}$ However, entering into details, the crystals oriented between $(2 \overline{1} 1)[0 \overline{1}]$ and $(0 \overline{1} 1)[0 \overline{1}]^{5)}$ have a tendency to rotate towards the $(2 \overline{1} 1)[0 \overline{1} \bar{l}]$ orientation.
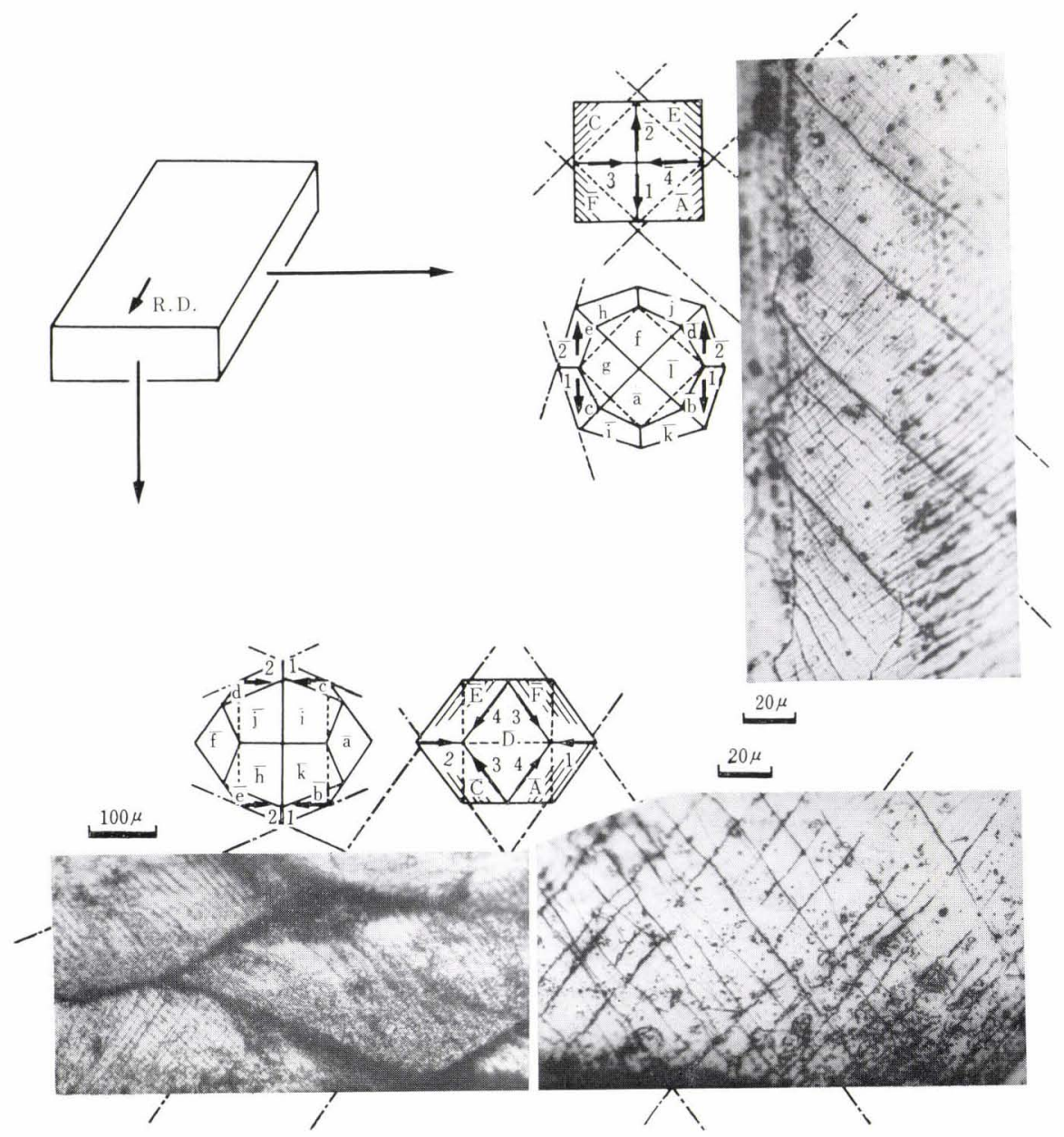

Fig. 32.

Slip patterns on front and side surfaces

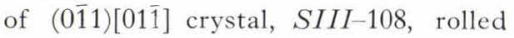
to $6 \%$ reduction 


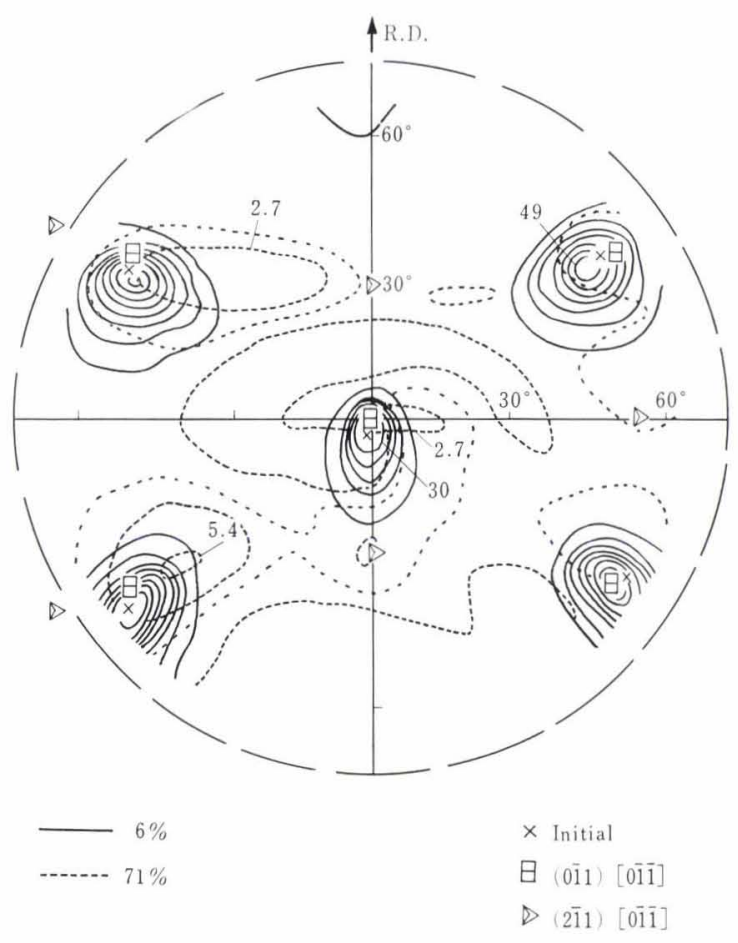

Fig. 33. (110) pole figure of $(0 \overline{1} 1)[0 \overline{1} \overline{1}]$ crystal, $S I I I-108$, rolled to 6 and $71 \%$ reduction

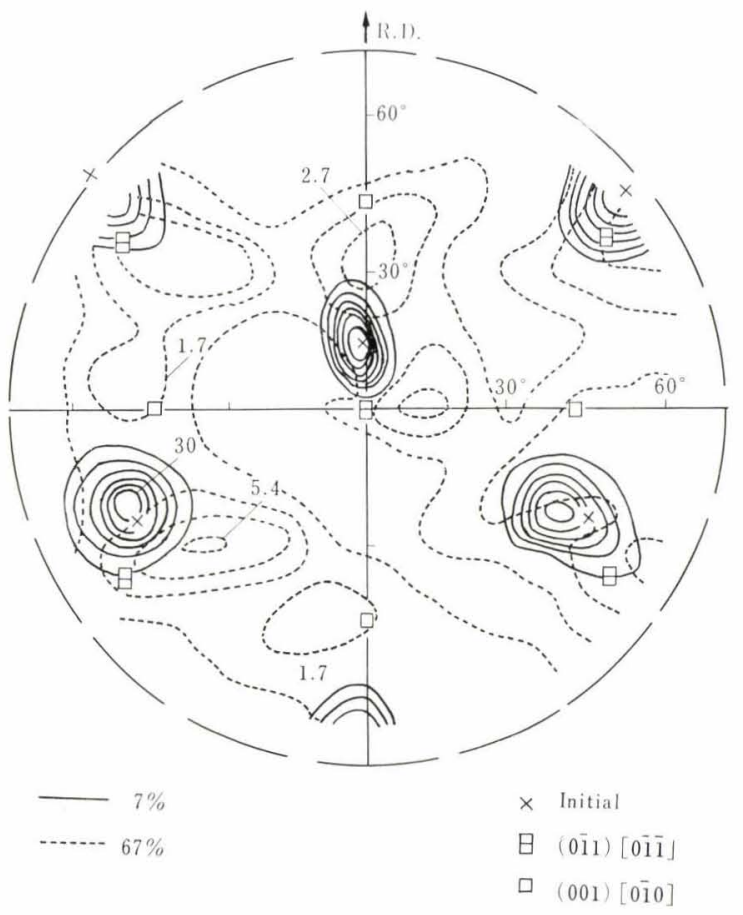

Fig. 34. (110) pole figure of $(0 \overline{3} 5)[0 \overline{5} \overline{3}]$ crystal, $S I I I-30$ rolled to 7 and $67 \%$ reduction

\section{Discussion}

In the previous section, the changes in texture with reduction have been described together with the operating slip planes, the microstructures, the average rolling pressure and the Vickers microhardness at various stages of rolling. It has been found how the rolling texture of single crystals with various initial orientations approaches either of the stable end orien- tations. As proposed by Barrett and Levenson, ${ }^{1)}$ the stable end orientations can be devided into two groups; namely, the first comprises the orientations having a $\{111\}$ plane parallel to the rolling plane,

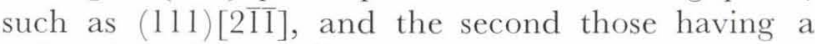
$\langle 011\rangle$ direction parallel to the rolling direction, such as $(100)[0 \overline{1} \overline{1}]$ or $(2 \overline{1} 1)[0 \overline{1}]$. In this section, the change in rolling textures towards the stable end orientations will be semi-quantitatively discussed taking the change of operative slip systems accompanying the rotation of the crystal.

The stress system under rolling, when simplified, can be regarded as bi-axial, i.e., tension along the rolling direction and compression along the rolling plane normal. This simplification may be rationalized when the diameter of working roll is large as compared with the strip thickness as in the present experiments.

Under a uniaxial stress in [uvw] direction, $\sigma_{\mathrm{uvw}}$, the resolved shear stress in a direction $\langle 111\rangle$ on a $\{\mathrm{hkl}\}$ plane, $\tau_{\mathrm{hk}}$, is generally given by

$$
\tau_{\mathrm{hki}}=\sigma_{\mathrm{uvW}} \cos \phi \cos \lambda=\sigma_{\mathrm{uvw}} / \mathbf{g}
$$

or

$$
\sigma_{\mathrm{uvw}}=\tau_{\mathrm{hk} 1} / \cos \phi \cos \lambda=\tau_{\mathrm{hk} 1} \cdot \mathbf{g}
$$

where $\phi$ is the angle between the normal to the slip plane and the axis of stress, either tension or compression, and $\lambda$ is the angle between the slip direction and axis of stress. The ' $\mathbf{g}$ ' is the reciprocal of Schmid factor, which is sometimes called the Taylor factor. The smaller the $\mathbf{g} \cdot \mathrm{S}_{\mathrm{hk} 1}$ value, the more operative is the slip system, where $S_{11 k 1}$ is the critical shear stress resolved on the $\{h \mathrm{hl}\}$ plane. It was reported on $3 \%$ silicon iron single crystals that two types of slip system actually operated under a simple tension, where the critical resolved shear stresses at room temperature were $13.4 \mathrm{~kg} / \mathrm{mm}^{2}$ for $\{110\}\langle 111\rangle$ systems, and 14.0 or $14.8 \mathrm{~kg} / \mathrm{mm}^{2}$ for $\{112\}\langle 111\rangle$ systems depending on the sense of slip direction. ${ }^{18)}$ As the difference between them amounts to only one tenth of each value, the operation of slip systems may be attributed to their g values.

However, the $\tau_{\mathrm{hk} 1}$ during rolling is a complex function of time and space; it changes with time at a given point, changes from a point to point at a given time. It is very difficult to express the $\tau_{\mathrm{hkl}}$ as a unique function of time and space, and to determine the operating slip systems during rolling. In practice, it is important to examine the mainly operating slip systems to understand the rotation of the crystal in rolling. When only one slip system operates, the crystal should so rotate geometrically that the slip direction approaches the rolling direction and the slip plane the rolling plane. ${ }^{25), 26)}$ General expression of the rotation of orientation as a function of shear strain on slip systems is given in Appendix II. Assuming that the slip systems observed to have operated in early stage continue to operate to later stage, it can be derived by simple geometrical consideration, how the rolling texture is developed by rotation with increasing reduction. 


\section{Slip Systems and Formation of Rolling Textures in SI}

In the (001)[100] crystal, the rolling texture was composed of two symmetrical components which were related to (001)[100] by rotation in both clockwise and counterclockwise directions around the normal to the rolling plane, see Figs. 4-6. In early stage, the slip bands were observed to be parallel to the traces of $\{110\}$ planes, A, C, as presented in Fig. 3 .

Concerning the family of slip systems, $\{110\}\langle 111\rangle$, the smallest $\mathbf{g}$ value is 2.45 and the same for four slip systems, A1, A4, C2, C3, in both tension and compression. The $\mathbf{g}$ values of the other eight systems $\{110\}$ $\langle 111\rangle$ are infinity in either tension or compression. Then the shear stress is the largest in the former four systems. With regard to the family of slip systems $\{112\}\langle 111\rangle$, the $\mathbf{g}$ value in compression is the smallest, 2.12 for four slip systems, $b 1, e 2, h 3, k 4$, while that in tension is very large, 4.23 , for them. In tension, the g value is the smallest, 2.12, for systems $a 1, f 2, g 3, l 4$, but is large, 4.23, in compression. Considering a stress system with tension and compression combined, it is thus understood that the operating slip planes had been the $A$ and $C$ of $\{110\}$.

It may be reasoned that the compression stress component predominates in cold rolling because the friction is small; the situation is further intensified when the diameter of working rolls is large. Then, as far as the compression stress is considered, once a system, $A 1$, begins to operate, its action becomes ever the harder to continue to operate as the rotation of the crystal progresses, and another system, C3, is instigated. Therefore, two systems, $A 1$ and C3, must always be considered to operate in pair. Thus, the (001) plane is maintained to be parallel to the rolling plane. The operation of this pair makes the crystal rotate clockwise around the [001] axis, while, the operation of the other pair, $A 4$ and C2, rotates the crystal counterclockwise in the similar way as the former.

When the tensile stress is brought into consideration along with the compression, the clockwise rotation by the A1-C3 pair makes the same pair more easily operative, while making the operation of the other pair, A4-C2, harder, as presented in Fig. 35. These stability criteria were first suggested by Tucker ${ }^{27)}$. Fig. 35 presents the $\mathbf{g}$ value of most of slip systems as a function of the rotation angle measured in clockwise direction from (001)[100] orientation around [001] axis. Arrows on the curves indicate the direction of rotation by operation of the pairing slip systems. The $\mathbf{g}$ value of $A 1, C 3$ in tension decreases with rotation to the minimum of 2.03 at $22.5^{\circ}$ and increases to the original value of 2.45 at (001) [110]. The $\mathbf{g}$ value in tension of $A 4, C 2$ is larger than that of $A 1, C 3$ in all the range, increasing up to infinity at (001)[110]. Then the clockwise rotation of the crystal by the A1-C3 pair continues on to $(001)[110]$ orientation, while the operation of the A4-C2 pair makes the crystal rotate counterclockwise. The independent operation of each

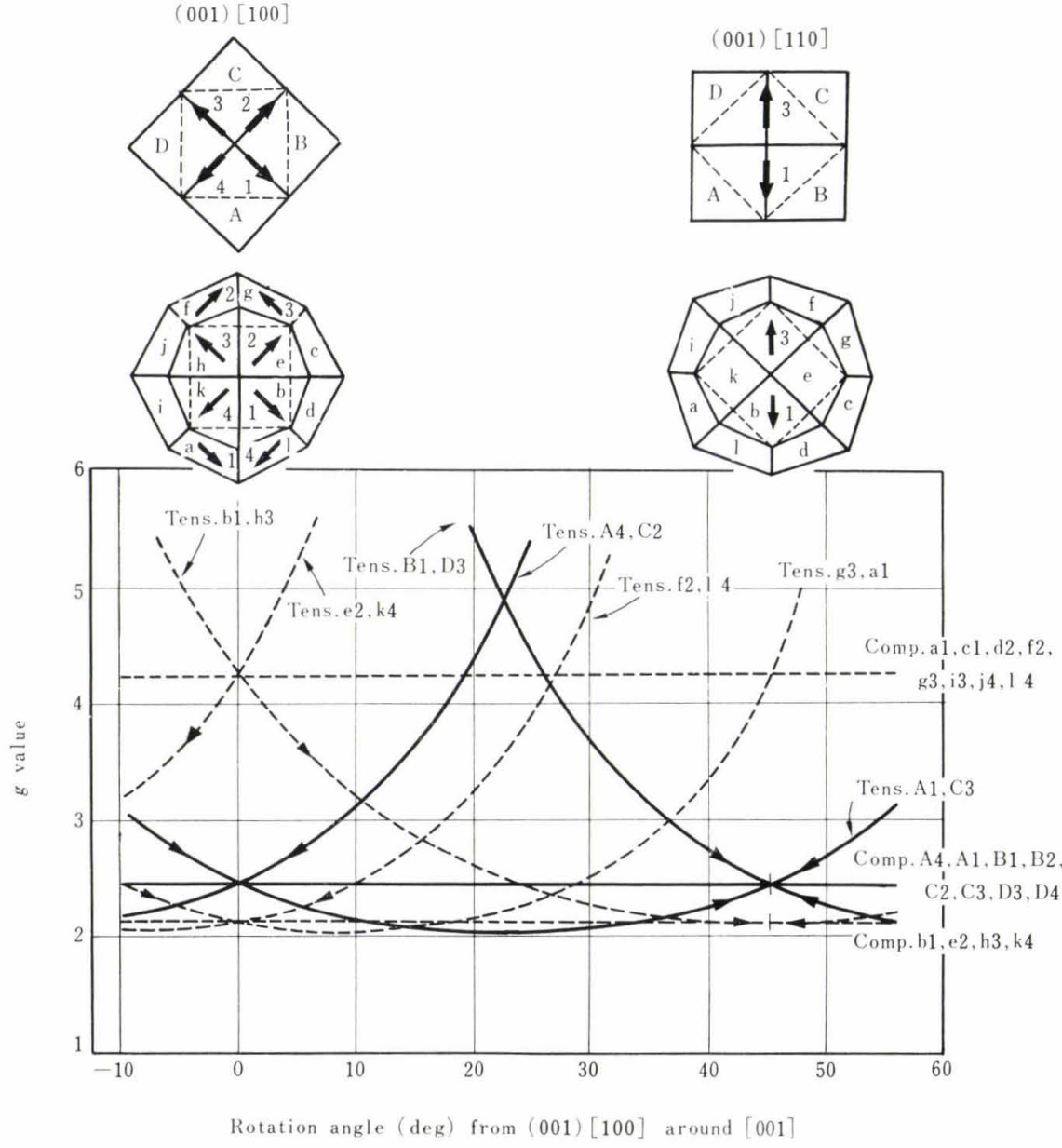

Fig. 35 .

Dependence of $\mathbf{g}$ value of representative $\{110\}\langle 111\rangle$ and $\{112\}\langle 111\rangle$ slip systems on rotation angle from (001)[100] orientation around [001] axis. Arrows put on curves indicate direction of rotation by operation of corresponding slip system. 
pair of slip systems in separated regions forms the two components of the rolling texture. Because of the strong friction between the roll and specimen surface in the transverse direction, it is difficult for one component to occupy a wide region in the transverse direction. And then, the banded structure will be formed along the rolling direction, and in fact observed as shown in Photo 1.

According to this simple deformation mechanism, the rotation of each component of the rolling texture with reduction, Fig. 6, may be calculated as follows. The original single crystal with (001)[100] orientation, in the shape of a unit square, OABC, is transformed into the shape of a parallelogram, $\mathrm{OAB}^{\prime} \mathrm{C}^{\prime}$, by operation of the $A 4-C 2$ pair in such a way that $\mathrm{C}$ and $\mathrm{B}$ move to $\mathrm{C}^{\prime}$ and $\mathrm{B}^{\prime}$, respectively, as shown in Fig. 36 (a). The parallelogramic deformation was clearly observed at the front edge of specimen as shown in Fig. 36 (b). After the deformation, the new rolling direction becomes parallel to $\mathrm{OC}^{\prime}$. The rotation angle, $\theta$, of the crystal is graphically calculated as a function of reduction, $X$, and is presented by a thin
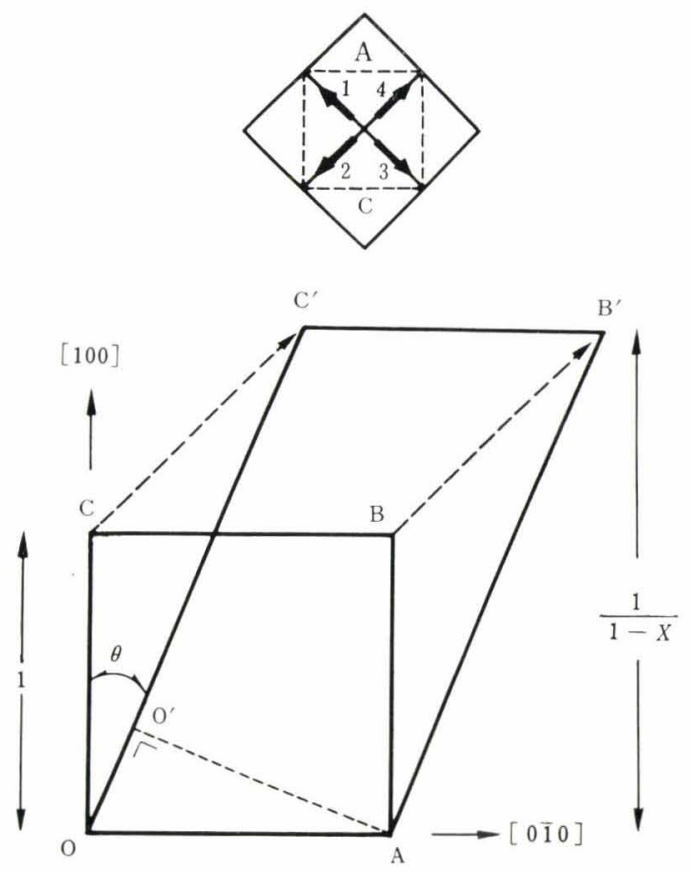

( a )

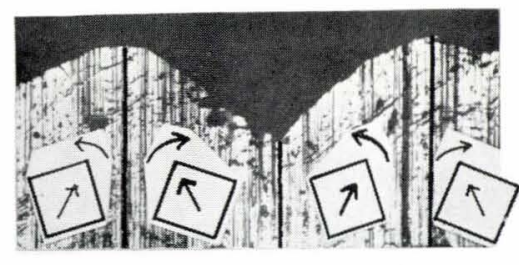

( b )

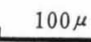

Fig. 36. (a) Schematic illustration of change of shape in (001)[100] crystal due to reduction by rolling

(b) Photograph of the front edge of (001) [100] crystal, $S I-77$, rolled to $75 \%$ reduction line in Fig. 6, together with the observed values. The observed values do not fall on the calculated curve. This is to be attributed to the simultaneous operation of the two pairs of slip systems in the same region because the possibilities of operation of the two pairs are the same at an early stage of reduction. If operation of any one pair becomes predominant at any subsequent stage of reduction, then the deformation will be carried on by that pair only. The thick line presents the calculated curve assuming that only one pair continues to be operative from a point indicated by $X$ at $51 \%$. The observed values are in agreement with this calculated curve.

According to Fig. 36, the width of specimen must decrease from $\mathrm{OA}$ to $\mathrm{O}^{\prime} \mathrm{A}$ by deformation, as actually observed in the (001)[410] crystal rolled to $73 \%$ reduction. In this crystal, the rotation was in one direction as a result of the operation of only one pair of slip systems, see Table 2. While in the (001)[100] crystal, this effect was eliminated by the simultaneous operation of two pairs.

It is naturally inferred from Fig. 35 that the (001) [110] crystal is in a stable end orientation for rolling. In the crystal, the mainly operating slip planes were found to be $\{110\}, A, B, C, D$, and $\{112\}, b, h$. Those crystals that deviated from the (001)[110] orientation rotate to the (001)[110] by the operation of the pair $A 1-C 3$ or by the other pair $B 1-D 3$, depending on the direction of deviation. Similar relation holds in the case of a pair of $\{112\}\langle 111\rangle$ systems, $b 1$ and $h 3$. Thus, it is concluded that the (001)[110] crystal is as stable for the rotation around the specimen normal, [001], as around the transverse direction.

Furthermore, the change of rolling texture in crystals oriented near (001)[100], such as (015)[100] and

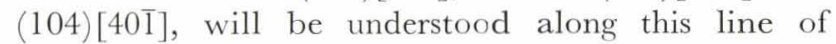
reasoning.

\section{Slip Systems and Formation of Rolling Textures in SII}

In the (011)[100] crystal, the rolling texture was composed of two symmetrical components which are related to the (011)[100] by rotation in both directions around the transverse direction, the two com-

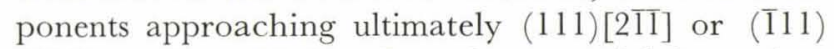
[211], see Figs. 16-18. In early stage of deformation, slip bands were observed to be parallel to the traces of $\{110\}, A, C, E, F$, or $\{112\}, a, f$, as observed in Fig. 15.

In the family of $\{110\}\langle 111\rangle$ slip systems, the $\mathbf{g}$ value is the smallest, 2.45 , for four slip systems, $A 1$, $F 1, C 2, E 2$, while in $\{112\}\langle 111\rangle$ slip systems, the $\mathbf{g}$ value is the smallest, 2.12, for two systems, $a 1, f 2$, all for both tension and compression. Comparing $2.45 \times \mathrm{S}_{110}$ to $2.12 \times \mathrm{S}_{112}$, it is probable that the two $\{112\}$ $\langle 111\rangle$ slip systems are more operative than $\{110\}\langle 111\rangle$ in early stage of rolling. The coarse slip bands along traces of $\{110\}$ planes as well as those of $\{112\}$ planes observed in Fig. 15 will be due to complex deformation on the front surface.

The operation of a slip system, al in direction 1, makes the crystal rotate upwards around the transverse $[0 \overline{1} 1]$ axis, while the operation of the other, $f 2$ 

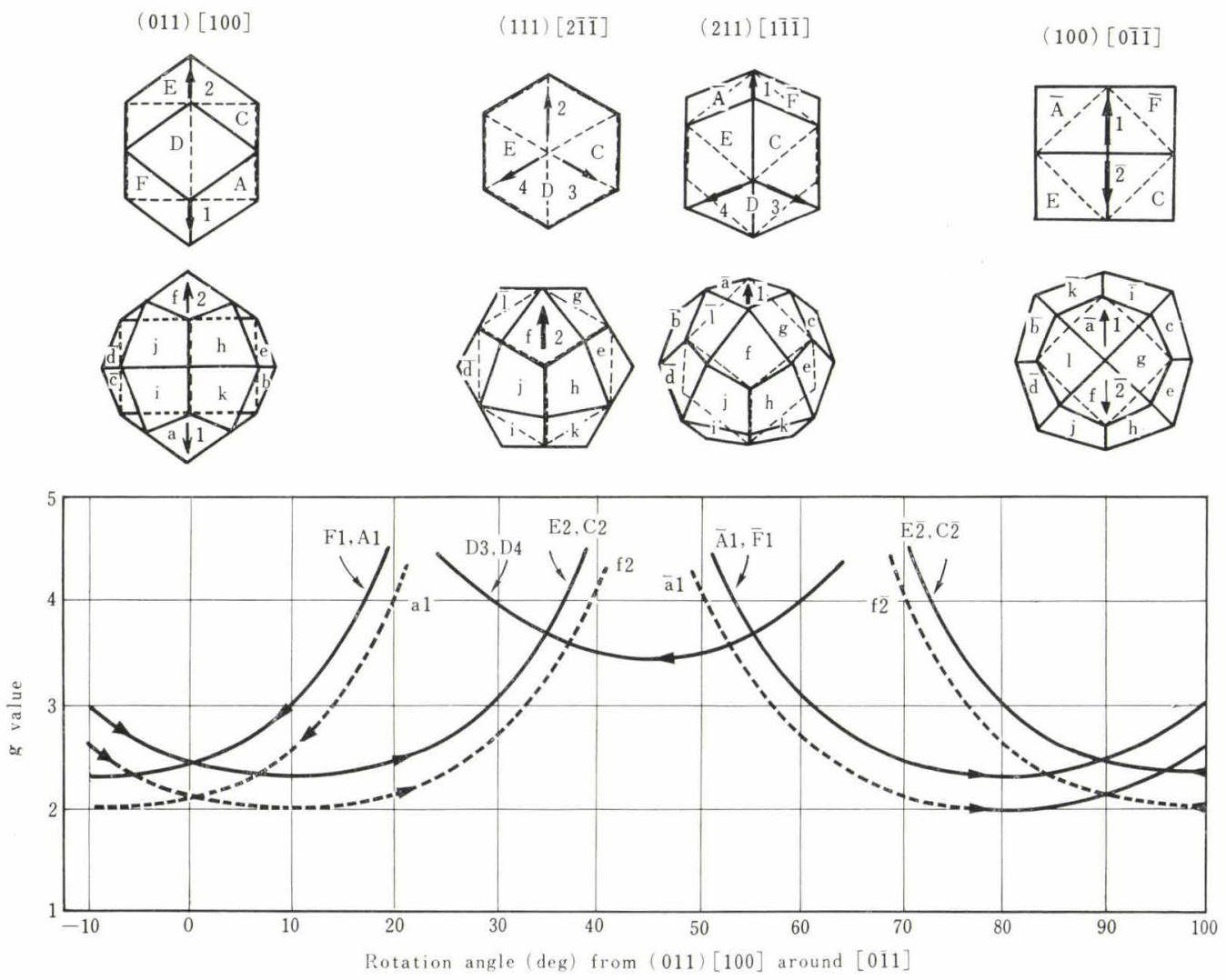

Fig. 37.

Dependence of $\mathbf{g}$ value of $\{110\}\langle 111\rangle$ and $\{112\}$ $\langle 111\rangle$ slip systems having small $\mathbf{g}$ values on rotation angle from (011)[100] orientation around transverse direction [011]

in direction 2, makes it rotate downwards. The change of $\mathbf{g}$ values of slip systems as the downward rotation of the crystal is presented in Fig. 37. The $\mathbf{g}$ values of slip systems shown in this figure are the same for both tension and compression in any orientation. The dotted curves for $f 2$ and $a 1$ indicate that the downward rotation by $f 2$ makes the $\mathbf{g}$ value of the system smaller than that of al system, and the crystal tends to rotate further in that direction. In the same way, the upward rotation by al makes the crystal rotate further in that direction, i.e., the (011) [100] orientation is unstable for rolling. Thus, two independent rotations in the respective two directions occur in different regions of the crystal to make the texture split into two components, as observed in Fig. 16 and Photo 3. The rotation in each direction will continue up to near $\{111\}<112\rangle$ orientation, though the $\mathbf{g}$ value of the operating system increases to 3.5 via the minimum of 2.0 at about $10^{\circ}$. As soon as the crystal rotates beyond the (111)[2\%], the $\mathbf{g}$ value of $f 2$ becomes larger than that of the other systems. Among these systems, $D \overline{3}$ and $D 4$ have small $\mathbf{g}$ values for both compression and tension, and in fact, in the (111)[211] crystal the slip bands along the traces of $D$ plane were observed, as shown in Fig. 26. The operation of $D \overline{3}$, D4 which are expected to act together, makes the crystal rotate upwards. Then, the rotation of the crystal stops near the (111)[2\%1] , and the orientation is maintained by the balanced operation of $D \overline{3}, D 4$ and $f 2$.

From the fact that the operating slip directions in the $(011)[100]$ crystal have no component in the transverse direction, there is no transverse friction between the roll and the specimen in contrast to the (001) [100] crystal. Thus, it is naturally understood that the regions of each component in the rolling texture were indefinite in shape and size in contrast to the banded structure in the (001) [100] crystal.

The independent operation of two slip systems in separated regions in early stage of rolling makes the calculation for the relation between the reduction and the rotation angle possible for each component. Under the operation of al or $f 2$ system, the relation is expressed by $X=\left\{\sin 55^{\circ}-\sin \left(55^{\circ}-\theta\right)\right\} / \sin 55^{\circ}$, where $X$ and $\theta$ are the reduction and the rotation angle, respectively, and $55^{\circ}$ is the angle between the slip direction and the rolling direction in the initial orientation. The relation is shown by a solid line in Fig. 18 together with the observed values. The observed values are in good agreement with the calculated curve up to $35^{\circ}$ rotation corresponding to $58 \%$ reduction, but beyond that reduction deviate from the calculated curve to keep a constant value of near the $35^{\circ}$ as anticipated from the above discussions.

In the (111) [2]1], $f 2$ and the pair of $D \overline{3}-D 4$ operate together because $\mathbf{g} \cdot \mathrm{S}$ value of $f 2$ is about the same as that of $D \overline{3}-D 4$ for both tension and compression. The crystal maintains its orientation by a balance of these three systems slipping in three different directions, $f 2, D \overline{3}, D 4$, as described before. However, it must be noted that the amount of slip in $f 2$ system has to be about 3.5 times that in $D \overline{3}$ or $D 4$ to keep the exact (111)[2]1] orientation, as shown in Appendix II. So, the stable orientation cannot be concluded to be exactly the (111)[2T1] orientation.

In the (211)[111] crystal, the rolling texture was 
composed of two components which was related to (211)[1i] by rotation in both directions around the transverse [0피 axis. In early stage of rolling, the slip bands were observed to be parallel to traces of $\{110\}, \bar{A}, D, \bar{F}$, and $\{112\}, \bar{a}$, as in Fig. 26. The observed $\bar{A} 1, \bar{F} 1, D \overline{3}, D 4$ and $\bar{a} 1$ systems have the smallest $\mathbf{g}$ values for each $\{110\}$ and $\{112\}$ systems for both tension and compression. The $\mathbf{g} \cdot \mathrm{S}$ values are almost the same for these slip systems. So, these slip systems are equally operative and the rotation of the crystal by slip in direction either 1 or $\overline{3}$ and 4 makes the slip geometrically easy to operate and the other hard, as understood from Fig. 37. Therefore, the rotation continues to the stable (100)[011] or (111)

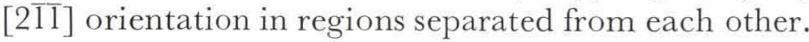

The asymmetrical distribution of the two components across the thickness in this crystal, Fig. 25, can be explained taking account of the difference of resultant stress applied in the upper and lower region of the specimen. As presented in Fig. 38, on the upper and lower surfaces of the specimen, the frictional stress, $\mathrm{F}$ and $\mathrm{F}^{\prime}$ act mainly in the direction shown in the figure, resulting in mutually opposite shear stress components in the upper and lower regions of the specimen. Thus, the resultant composite stress including the shear stress above is favourable for the operation of the slip system in direction 1 in the upper region and favourable for the systems in directions 3 and 4 in the lower. As a result, the (211)[111] crystal rotates to $(100)[0 \overline{1}]$ orientation in the upper region

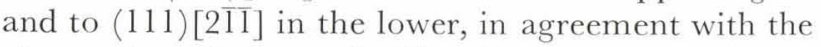
observed result shown in Fig. 25.

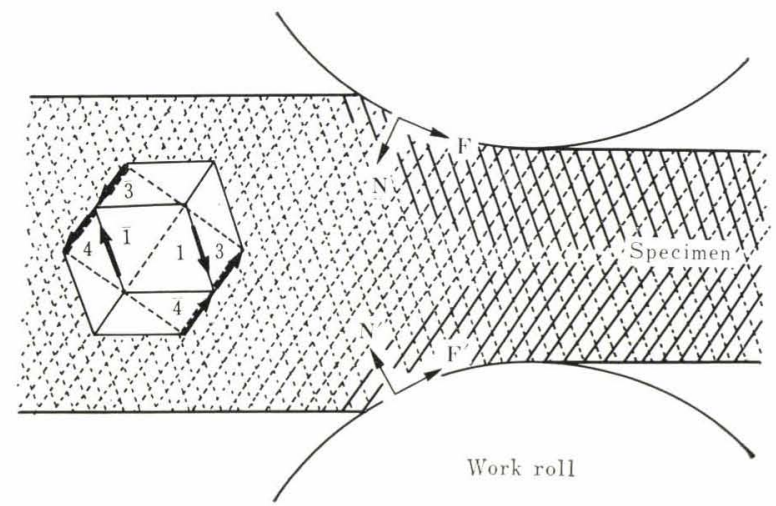

Fig. 38. Schematic illustration of operation of different slip systems in upper and lower regions of sheet of (211)[111] crystal under rolling

$\mathrm{F}$ and $\mathrm{F}^{\prime}$ : frictional stress

$\mathrm{N}$ and $\mathrm{N}^{\prime}$ : normal stress

Solid parallel lines : traces of operative slip planes

Taking the independent operation of slip systems in the upper and lower region into account, relations between the rotation angle of crystal and the amount of reduction can be readily calculated similarly as in the (011)[100] crystal. The calculated curves agree well with the observed values as shown in Fig. 24. As is expected, the rate of rotation decreases as the orientation approaches (100)[0]1] or (111)[2]1]. The (100)[0]1] orientation can easily be understood as stable for rotation around the transverse [011] direction owing to the balanced operation of slip systems in directions 1 and 2 .

Fig. 37 shows that in the crystals oriented between (011)[100] and (211) [111], only the rotation to (111)

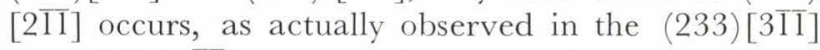
and $(322)[4 \overline{3} \overline{3}]$ crystals shown in Figs. 19 and 20, respectively. The crystals oriented between (211)

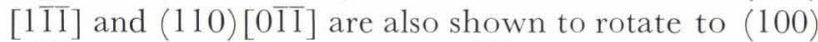

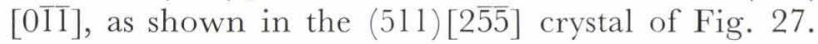

Thus it has been understood that the rolling textures of crystals in SII are formed by the rotation to either of the two stable end orientations, a $\{111\}\langle 211\rangle$ or a $\{100\}\langle 011\rangle$. The relations between the rotation angles and the reductions in thickness have been explained in good agreement with the observed results.

Further, the change of rolling texture in crystals oriented near the (011)[100], such as (025)[100], will be understood along this line of reasoning.

\section{Slip Systems and Formation of Rolling Textures in SIII}

All the crystals having a common [0T1] axis parallel to the rolling direction in SIII maintained their initial orientations almost unchanged through the rolling, though they tended to rotate more or less around the rolling direction. These behaviours may be understood from the change of $\mathbf{g}$ value with rotation of crystal around the rolling direction, see Fig. 39. In all the crystals of this series, the $\mathbf{g}$ values in tension for $\{110\}$ $\langle 111\rangle$ and $\{112\}\langle 111\rangle$ slip systems are kept constant, as presented in the figure. In the following, the authors will discuss only the effect of compression.

It has already been discussed that the (100)[011] crystal is stable for rotations around the transverse direction and the specimen normal. As for the rotation around the rolling direction, the orientation is stable when a pair of $\{112\}$ systems, $\bar{a} 1-f \overline{2}$, is in operation, but is unstable under the operation of either pair of $\{110\}$ systems, $\bar{A} 1-E \overline{2}$ or $C \overline{2}-\bar{F} 1$, as expected from Fig. 39. Considering the lower $\mathbf{g} \cdot \mathrm{S}$ value of the pair $\bar{a} 1-f \overline{2}$, it is naturally understood that the initial orientation was almost maintained. The lateral spread of the surface texture, Fig. 12, may have been caused by the operation of $\{110\}$, slip systems in the surface layer.

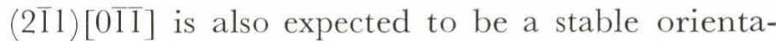
tion by the following two reasons. The two systems, $\bar{F} 1$ and $C \overline{2}$, with the smallest $\mathbf{g}$ value must operate in pair in the same way as described for the pair of $A 1$ C3 in the (001)[100] crystal. The operation in pair not only keeps the [0]1] direction parallel to the rolling direction, but also keeps the [1]1] direction parallel to the transverse direction, because the normals to the $\bar{F}$ and $C$ planes are perpendicular to the transverse direction, see Appendix II.

In the (1 $\overline{1} 1)[0 \overline{1}]$ crystal, the $\mathbf{g}$ values of $g 3, c 1$ and $e \overline{2}$ in compression are smaller as compared to those of $\bar{F} 1, C \overline{2}$, but the $\mathbf{g}$ values of the former systems are much larger in tension, as seen in Fig. 39. Under the

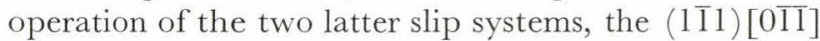
crystal has a tendency to rotate to $(2 \overline{1} 1)[0 \overline{1} \overline{1}]$ around 
(011) $[0 \overline{1} \overline{1}]$
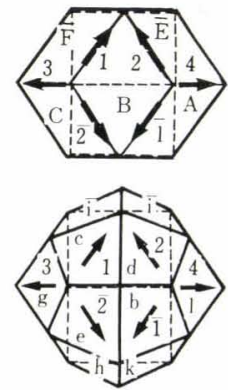

(11̄1) $[0 \overline{1} \overline{1}]$
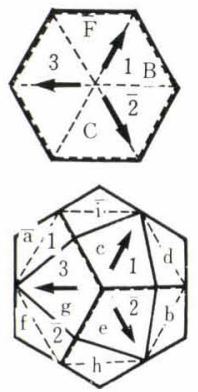

(2î1) $[0 \overline{1} \overline{1}]$
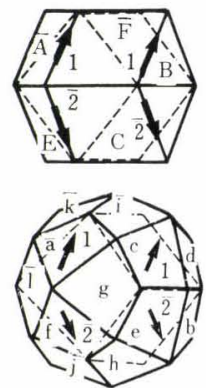

(100) [0 $\overline{1} \overline{1}]$
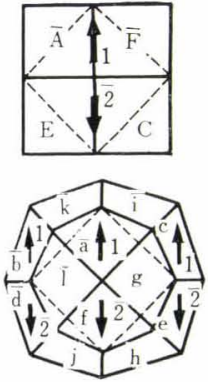

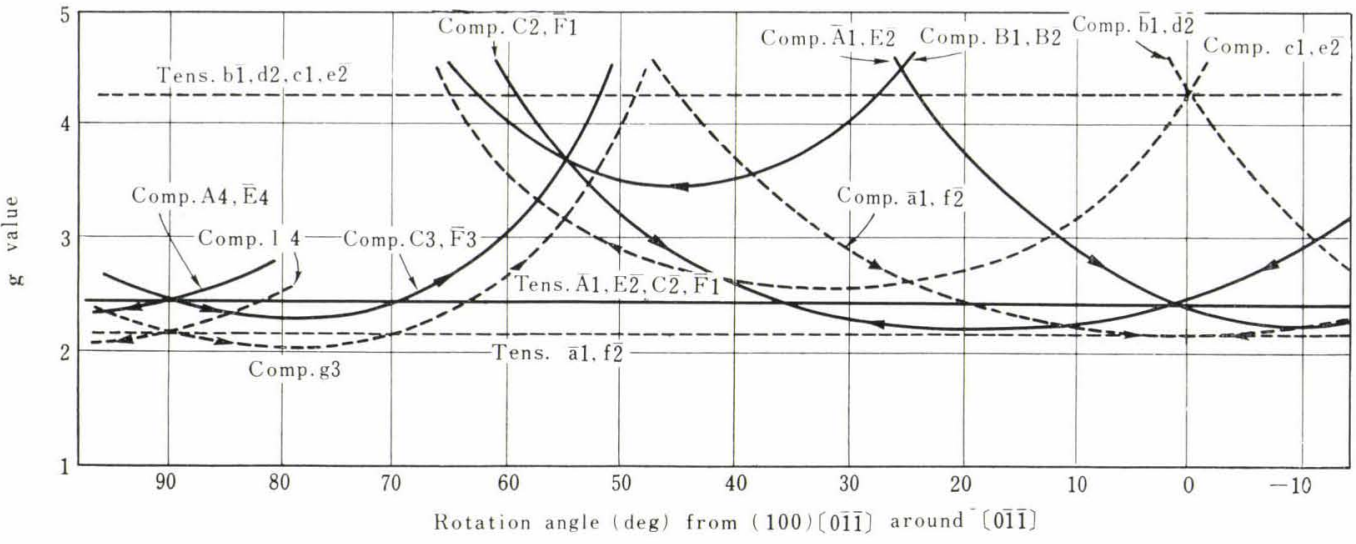

Fig. 39

Dependence of $\mathbf{g}$ value of representative $\{110\}\langle 111\rangle$ and $\{112\}\langle 111\rangle$ slip systems on rotation of crystals from $(100)[0 \overline{1} \overline{1}]$ to (01) $[0 \overline{1} \overline{1}]$ around rolling direction $[0 \overline{1} \overline{1}]$

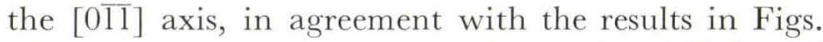
29 and 30, but in contrast to the results of Koh and Dunn. ${ }^{4}$

Finally, the $(0 \overline{1} 1)[0 \overline{1} \overline{1}]$ crystal is hard to deform by rolling because all the slip systems have infinite $\mathbf{g}$ value either for compression or tension. Now, the slip systems, g3, $C 3, \bar{F} 3$, or $14, A 4, \bar{E} 4$, must operate to reduce the specimen thickness, in spite of the directions 3 and 4 being perpendicular to the rolling direction, resulting in widening the crystal in early stage of rolling as observed. The operation of the slip systems in the direction 3 or 4 makes the crystal continue to rotate in right or left direction and the texture is spread, as shown in Fig. 33. Furthermore, systems $\bar{F} 1, A \overline{1}, C \overline{2}, \bar{E} 2$ need to operate in order to elongate the specimen while decreasing the width. However, the simultaneous operation of these four systems has no effect on the orientation change of the crystal.

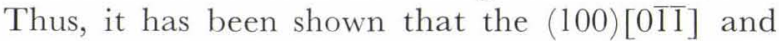

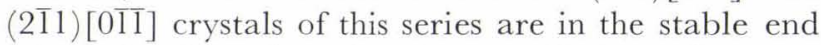
orientation but the others have a little tendency to rotate to either of the two. This is in disagreement with other reports in some detail.4)

Summarizing the above discussions, the formation of rolling textures has been systematically explained for all the crystals with typical orientations from the consideration of the operating slip systems. Rolling texture of any crystal in an arbitrary orientation could be described in a similar way as that given to the typical crystal with the nearest orientation. The stable orientations are thus those three, namely $\{100\}\langle 011\rangle$, $\{111\}\langle 211\rangle$ and $\{211\}\langle 011\rangle$, though in order for the former two orientations to be stable, some restrictions are required for the operation of slip.

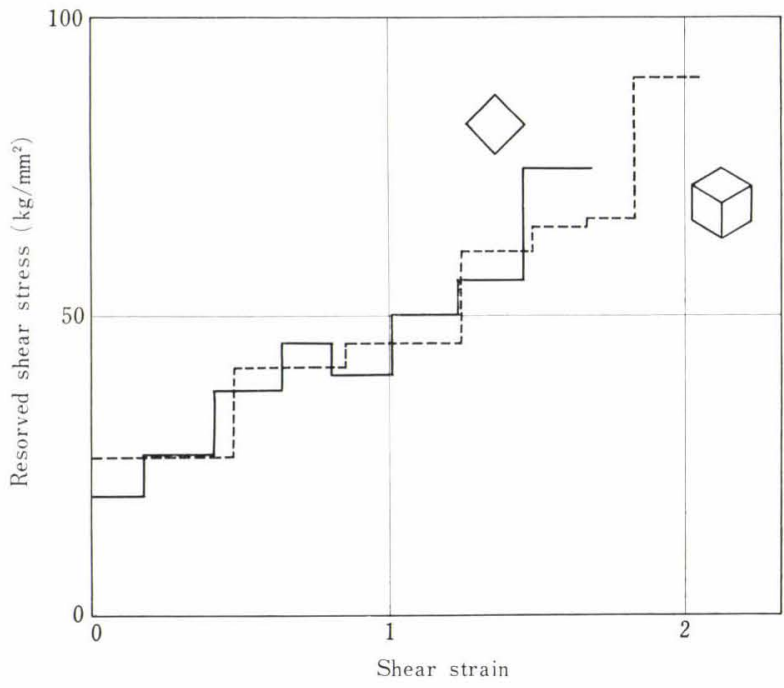

Fig. 40. Shear stress-shear strain curve resolved on operating slip systems in (001)[110] and (111)[21̄1] crystal, calculated from relation of average rolling pressure versus reduction

\section{Miscellanea}

The average rolling pressure versus reduction curves of Figs. 2 and 14 can be reduced to the resolved shear stress versus shear strain curves (r.s.s.-s.s. curves) on the operating slip systems, as indicated in Fig. 40. There is not much difference between the r.s.s.-s.s. curve resolved on $\{110\}$ plane of the (001) [110] crystal and that on $\{112\}$ plane of another oriented near (111) $[2 \overline{11}]$, though the apparent pressure curves of the two are remarkably different. The fact that the apparent

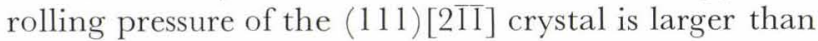
that of the (001)[110] crystal at the same reduction in 
thickness is attributed to the higher shear strain in the former as compared to that in the latter. It is concluded that the apparent rolling pressure versus reduction curve is strongly affected by the orientation of crystals. The fact is more marked in the apparent curve in the (011)[100] crystal in which the stress decreases with strain, see Fig. 14 (a). This is attributed to the decrease of the $\mathbf{g}$ value due to the rotation of the crystal, as presented in Fig. 37.

In the subsequent recrystallization of rolled crystals, the substructures as well as the texture play an important role. On the substructures described in this Part I, discussion will be given in Part II in relation to the recrystallization. Recovery and recrystallization take place though some stages evolving the internal energy. Discussions will also be given on the average rolling pressure and Vickers hardness as a measure of the internal energy relating to the recrystallization rate.

\section{Acknowledgements}

It is a pleasure to thank Director U. Hashimoto and Professor S. Kaya for their support and encouragement throughout this work. The authors wish to thank Dr. T. Takeuchi, Messrs. M. Ohkochi and K. Yagisawa for their cooperation in the early periods of this study and Messrs. Y. Saito and H. Hayakawa for their assistance in specimen preparation and measurements. They would also like to thank Drs. R. Honda, A. Yoshikawa and other staffs in Metal Physics Division of NRIM and Messrs. J. Imamura and K. Suzuki for their valuable discussions.

\section{REFERENCES}

1) C. S. Barrett and L. H. Levenson: Trans. AIME, 145 281.

2) B. F. Decker and D. Harker: J. Appl. Phys., 22 (1951), 900.

3) G. G. Dunn: Acta Met., 2 (1954), 173.

4) P. K. Koh and G. G. Dunn: Trans. AIME, 203 (1955), 401.

5) J. L. Walter and W. R. Hibbard: Trans. AIME, 212 (1958), 731.

6) H. Hu: Acta Met., $8(1960), 124$.

7) H. Hu: Trans. AIME, 221 (1961), 130.

8) C. G. Dunn and P. K. Koh: Trans. AIME, 206 (1956), 1017.

9) H. Hu: Trans. AIME, 215 (1959), 320.

10) J. L. Walter, W. R. Hibbard and C. G. Dunn: Acta Met., $8(1960), 126$.

11) C. G. Dunn and H. Hu: Trans. AIME, 221 (1961), 878.

12) H. Hu: Recovery and Recrystallization of Metals, (1962), 311, [edited by L. Himmel, Interscience Publishers].

13) H. Hu: Trans. AIME, 224 (1962), 75.

14) H. Hu: Acta Met., 10 (1962), 1112.

15) H. Hu: Trans. AIME, 230 (1964), 572.

16) J. L. Walter and E. F. Koch: Acta Met., 11 (1963), 923.

17) J. L. Walter and E. F. Koch: Trans. AIME, 233 (1965), 1209.

18) T. Taoka, S. Takeuchi and E. Furubayashi: J. Phys. Soc. Japan, 19 (1964), 701.

19) C. E. Morris: Metal Progress, 56 (1949), 691.

20) G. Bassi : Z. Metallkde., 51 (1960), 357.

21) T. Taoka, E. Furubayashi and S. Takeuchi : Japanese J.
Appl. Phys., 4 (1965), 120.

22) H. Abe, S. Matsuo and K. Ito: J. Japan Inst. Metals, 26 (1962), 684 (in Japanese).

$23)$ C. G. Dunn: Trans. AIME, 167 (1946), 373.

24) H. Hu and R. S. Cline: Trans. AIME, 224 (1962), 784.

25) E. A. Calnan and C. J. B. Clews: Phil. Mag., 42 (1951), 616.

26) I. L. Dillamore and W. T. Roberts: Acta Met., 12 (1964), 281.

27 G. E. G. Tucker: J. Inst. Met., 82 (1954), 655.

\section{Appendix I:}

Notation of slip directions and slip planes.

In Fig. 41, the slip directions and poles of operative slip planes are indicated on the standard (001) stereographic projections for body-centred cubic crystals. The $\{111\}$ slip directions are indicated by figures $\bar{I}-\overline{4}$ while the poles of the $\{110\}$ slip planes are indicated by capital letters $A, B, C, D, E$ and $F$, and those of the $\{112\}$ slip planes by small letters $a, b, c, d, e$, $f, g, h, i, j, k$ and $l$. Poles in the opposite directions are distinguished from each other by minus sign such as $l$ and $\bar{I}, A$ and $\bar{A}$, and $a$ and $\bar{a}$. Slip systems are expressed by combination of notations of plane and direction, such as $A 1, a 2$.

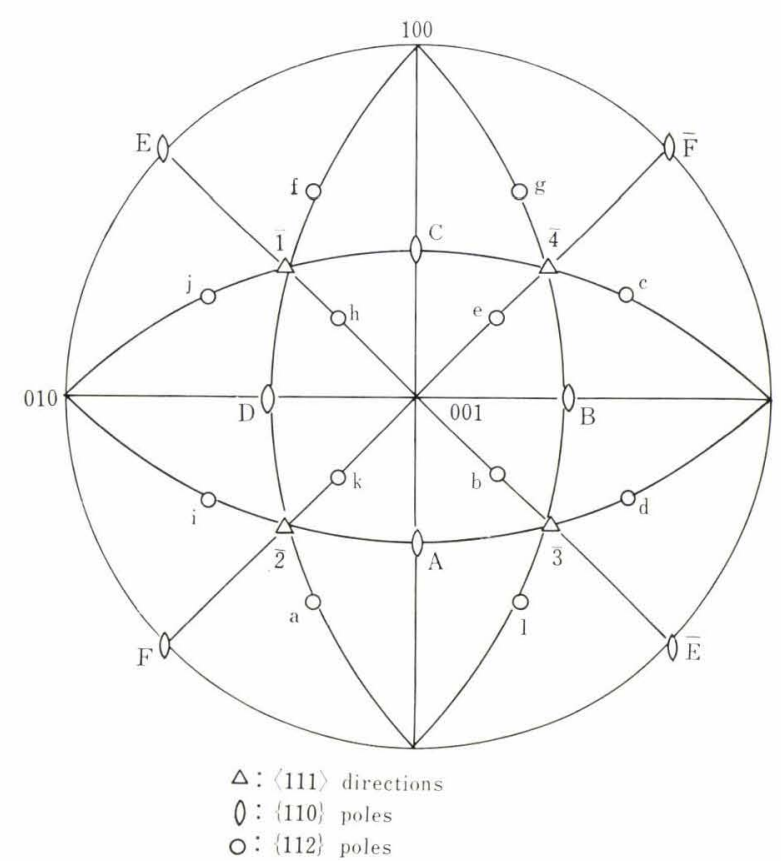

Fig. 41. Standard (001) stereographic projection for bcc crystals showing slip directions, $\langle 111\rangle$, and poles of slip planes, $\{110\}$ and $\{112\}$

\section{Appendix II:}

Change of crystal shape and orientation as a function of shear strain of active slip systems.

Consider a unit cube in the original crystal, three sides of which, $\mathbf{R}, \mathbf{T}, \mathbf{N}$, are parallel to rolling, transverse and normal direction, respectively. When shearing the specimen by a single slip system with direction $\mathbf{D}$ and plane $\mathbf{P}$, the unit cube deforms into a parallelepiped that the unit vectors, $\mathbf{R}, \mathbf{T}$, and $\mathbf{N}$, that difine the unit cube in reference to the particular 


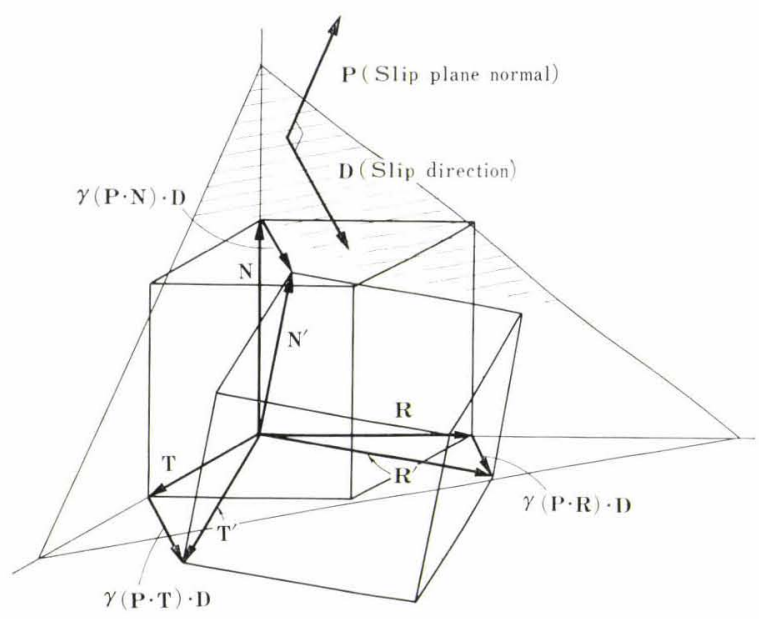

Fig. 42. Schematic illustration showing change in orientation of rolling plane and rolling directions owing to operation of a single slip system

crystallographic orientation, are converted into new corresponding vectors, $\quad \mathbf{R}^{\prime}=\mathbf{R}+\gamma(\mathbf{P} \cdot \mathbf{R}) \mathbf{D}, \quad \mathbf{T}^{\prime}=\mathbf{T}+$ $\gamma(\mathbf{P} \cdot \mathbf{T}) \mathbf{D}$, and $\mathbf{N}^{\prime}=\mathbf{N}+\gamma(\mathbf{P} \cdot \mathbf{N}) \mathbf{D}$, where $\gamma$ is the shear strain of the slip system, as shown in Fig. 42. New rolling direction is now changed from $\mathbf{R}$ to $\mathbf{R}^{\prime}$, while the rolling plane normal from $\mathbf{N}$ to $\left[\mathbf{T}^{\prime} \times \mathbf{R}^{\prime}\right]=$ $\mathbf{N}+\gamma[[(\mathbf{P} \cdot \mathbf{R}) \mathbf{T}-(\mathbf{P} \cdot \mathbf{T}) \mathbf{R}] \times \mathbf{D}]$. For simultaneous operation of more than one system, the new rolling direction and the new rolling plane normal are parallel

$$
\text { to } \begin{aligned}
\mathbf{R} & +\int_{\mathrm{i}} \sum_{\mathrm{i}}\left(\mathbf{P}_{\mathrm{i}} \cdot \mathbf{R}\right) \mathbf{D}_{\mathrm{i}} d \gamma_{\mathrm{i}}, \text { and } \mathbf{N}+\int \sum_{\mathrm{i}}\left[\left[\left(\mathbf{P}_{\mathrm{i}} \cdot \mathbf{R}\right) \mathbf{T}\right.\right. \\
& \left.\left.-\left(\mathbf{P}_{\mathrm{i}} \cdot \mathbf{T}\right) \mathbf{R}\right] \times \mathbf{D}_{\mathrm{i}}\right] d \gamma_{\mathrm{i}},
\end{aligned}
$$

respectively.

Example 1: Stability of $(2 \overline{1} 1)[0 \overline{1}]$ orientation.

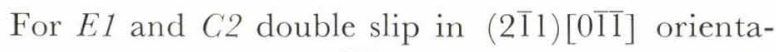
tion, put $\mathbf{N}=1 / \sqrt{6}[2 \overline{1} 1], \quad \mathbf{R}=1 / \sqrt{2}[0 \overline{1} \overline{1}]$, $\mathbf{T}=1 / \sqrt{3}[\overline{11} 1], \quad \mathbf{P}_{1}=1 / \sqrt{2}[1 \overline{1} 0], \quad \mathbf{D}_{1}=1 / \sqrt{3}[\overline{111}]$, $\mathbf{P}_{2}=1 / \sqrt{2}[101], \quad \mathbf{D}_{2}=1 / \sqrt{3}[\overline{1} 11], \quad$ and $\gamma_{1}=\gamma_{2}$. Then,

and

$$
\begin{aligned}
& \mathbf{N}+\int_{i} \sum_{i}\left[\left[\left(\mathbf{P}_{i} \cdot \mathbf{R}\right) \mathbf{T}-\left(\mathbf{P}_{i} \cdot \mathbf{T}\right) \mathbf{R}\right] \times \mathbf{D}_{i}\right] d \gamma_{i} \\
& \quad=\left(1 / \sqrt{6}-\gamma_{1} / 3\right)[2 \overline{1} 1] / / \mathbf{N}
\end{aligned}
$$

$$
\mathbf{R}+\int \sum_{i}\left(\mathbf{P}_{i} \cdot \mathbf{R}\right) \mathbf{D}_{i} d \gamma_{i}=\left(1 / \sqrt{2}+\gamma_{1} / \sqrt{3}\right)[0 \overline{1} \overline{1}] / / \mathbf{R}
$$

Thus, the $(2 \overline{1} 1)[0 \overline{1}]$ orientation is proved to be a stable orientation for the simultaneous operation of $\bar{F} 1$ and $C 2$ slips.

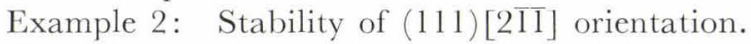

For $D \overline{3}, D 4$ and $f 2$ triple slip in (111) [2]1] orientation, put $\mathbf{N}=1 / \sqrt{ } 3[111], \quad \mathbf{R}=1 / \sqrt{6}[2 \overline{1} \overline{1}]$, $\mathbf{T}=1 / \sqrt{2}[0 \overline{1} 1], \quad \mathbf{P}_{1}=1 / \sqrt{2}[011], \quad \mathbf{D}_{1}=1 / \sqrt{3}[\overline{1} 1 \overline{1}]$, $\mathbf{P}_{2}=1 / \sqrt{2}[011], \quad \mathbf{D}_{2}=1 / \sqrt{3[111]}, \quad \mathbf{P}_{3}=1 / \sqrt{6}[211]$, $\mathbf{D}_{3}=1 / \sqrt{ } 3[1 \overline{11}]$, and the ratio of shear strain of $f 2$ slip to that of $D \overline{3}$ and $D 4$ equals $r$, or $d \gamma_{3}=r d \gamma_{2}=r d \gamma_{1}$.

Then,

$$
\begin{aligned}
\mathbf{N} & +\int \sum_{i}\left[\left[\left(\mathbf{P}_{\mathrm{i}} \cdot \mathbf{R}\right) \mathbf{T}-\left(\mathbf{P}_{\mathrm{i}} \cdot \mathbf{T}\right) \mathbf{R}\right] \times \mathbf{D}_{\mathrm{i}}\right] d \gamma_{\mathrm{i}} \\
& =1 / \sqrt{3}[111]+\gamma_{1} / 3 \sqrt{6}[2 r, r+2 \sqrt{3}, r+2 \sqrt{3}]
\end{aligned}
$$

and

$$
\begin{gathered}
\mathbf{R}+\int \sum_{\mathrm{i}}\left(\mathbf{P}_{\mathrm{i}} \cdot \mathbf{R}\right) \mathbf{D}_{\mathrm{i}} d \gamma_{\mathrm{i}} \\
=1 / \sqrt{6}[2 \overline{1} \overline{1}]+\gamma_{1} / 3 \sqrt{3}[r+2 \sqrt{3}-r-r]
\end{gathered}
$$

Thus, only when $r=2 \sqrt{3} \fallingdotseq 3.5$, the crystal can maintain its initial (111)[2]1] orientation. 